Skrandies, Wolfgang:

The upper and lower visual field of man : Electrophysiological and functional differences

Quelle: Progress in Sensory Physiology, Vol. 8, 1987, S. 1-93

Berlin: Springer-Verlag

Mit freundlicher Genehmigung von Springer Science and Business Media 


\title{
The Upper and Lower Visual Field of Man: Electrophysiological and Functional Differences*
}

\author{
W. Skrandies
}

Max-Planck-Institut für Physiologische und Klinische Forschung, W. G. Kerckhoff-Institut, 6350 Bad Nauheim, FRG

Introduction: The Divided Outer World

2 Analysis and Origin of Visual Evoked Potential Components .......

Topographical Analysis of Scalp-Recorded Brain Activity ...........

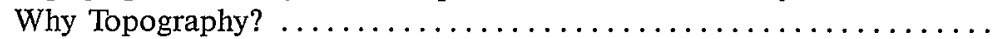

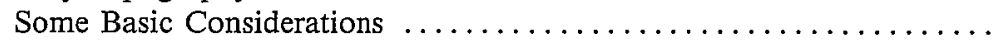

Origin of Visual Evoked Potential Components $\ldots \ldots \ldots \ldots \ldots \ldots \ldots \ldots, 15$
Temporal Activity Patterns $\ldots \ldots \ldots \ldots \ldots \ldots \ldots \ldots \ldots \ldots \ldots \ldots$

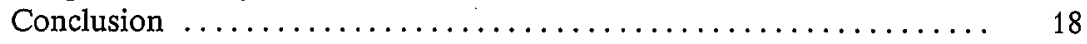

3 Differences in Latency and Topography of Visual Evoked Potential

3.1 Components .......................

Pattern Reversal Evoked Activity Recorded in 45 Channels ......... 20

3.2 Generalization and Extension of Visual Evoked Potential Findings ... 22

3.2.1 Pattern Reversal Stimuli ........................... 22

3.2.2 Different Presentation Modes: Onset, Offset, and Reversal ......... 26

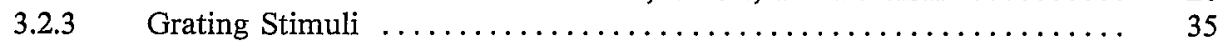

3.3 Implications for the Clinical Use of Visual Evoked Potentials ....... 38

4 Electrophysiological Differences at Other Stages of the Visual System 41

4.1 Retinal Pigment Epithelium Activity $\ldots \ldots \ldots \ldots \ldots \ldots \ldots \ldots \ldots . \ldots \ldots, 41$

$4.2 \quad$ Human Pattern Electroretinograms $\ldots \ldots \ldots \ldots \ldots \ldots \ldots \ldots \ldots . \ldots \ldots$

4.2.1 Spatial Distribution of Pattern Electroretinograms and Evoked Potentials 46

4.2.2 Differences Between Upper and Lower Retinal Areas ............. 49

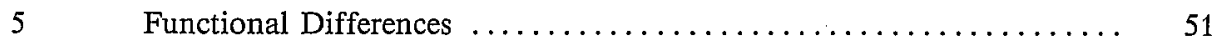

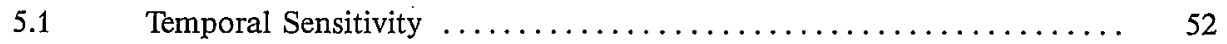

5.1.1 Results on Critical Flicker Fusion Frequency $\ldots \ldots \ldots \ldots \ldots \ldots \ldots, 53$

$5.1 .2 \quad$ Results on Double Flash Discrimination $\ldots \ldots \ldots \ldots \ldots \ldots \ldots \ldots . . \ldots \ldots$

5.1.3 Relation Between Critical Flicker Fusion Frequency and Relative

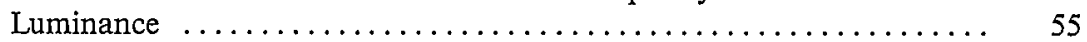

$5.2 \quad$ Visual Acuity and Contrast Sensitivity $\ldots \ldots \ldots \ldots \ldots \ldots \ldots \ldots . \ldots \ldots$

5.3 Discrimination of Meaningful Stimuli $\ldots \ldots \ldots \ldots \ldots \ldots \ldots \ldots . \ldots$

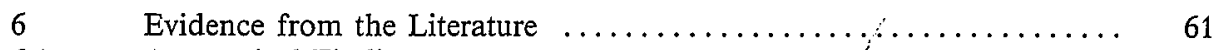

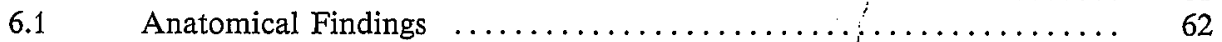

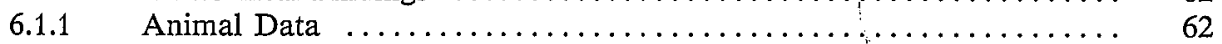

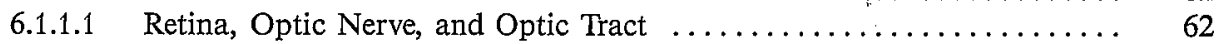

* To Annemarie and Gernot 


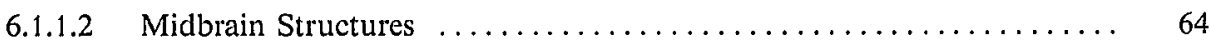

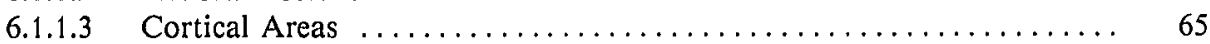

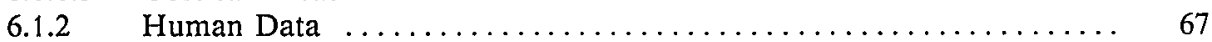

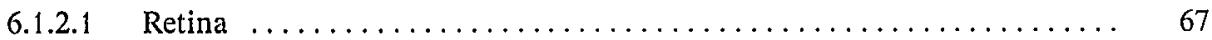

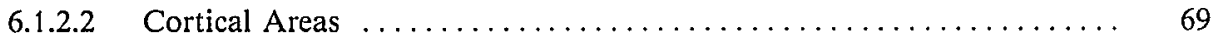

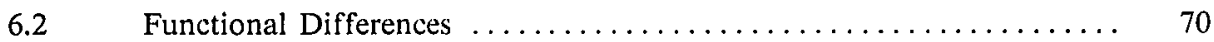

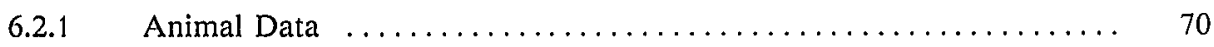

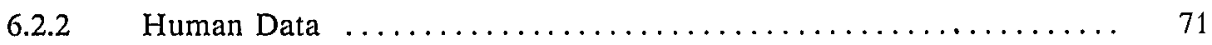

6.2.2.1 Motor Reaction Time to Visual Stimuli .................. 71

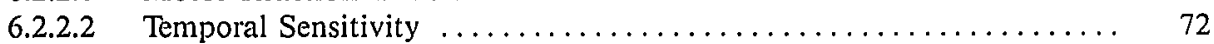

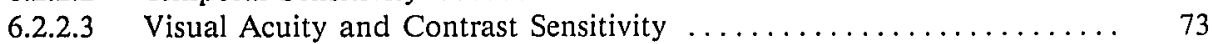

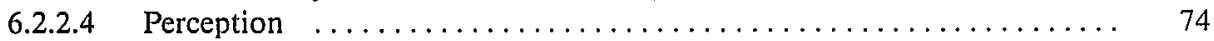

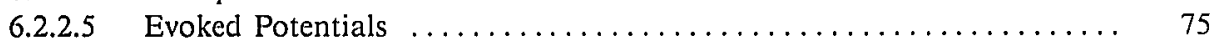

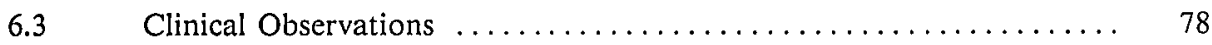

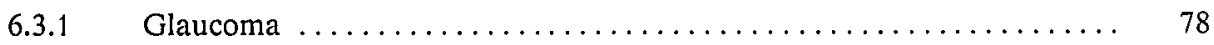

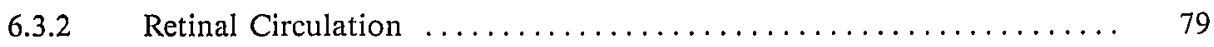

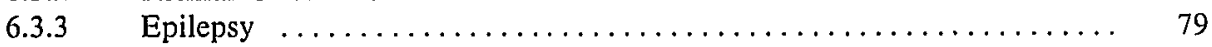

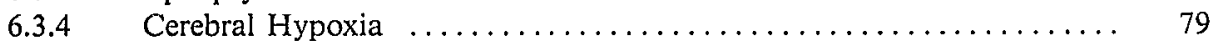

7 Summary and General Conclusions $\ldots \ldots \ldots \ldots \ldots \ldots \ldots \ldots . \ldots \ldots$

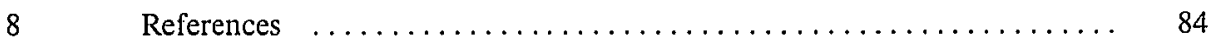

We are all aware of the fact that science has become more and more impatient and competitive. New experiments and new techniques may open new ways of understanding the nervous system. However, their success is based entirely on the intelligent and responsible use of them, which implies a thorough knowledge of what has been learned from the serious work of the past. The questions remain the same, and the techniques themselves should not be overvalued. Rather, the knowledge and results presented ... should be judged by the intelligence of the question, the seriousness of the approach, and the rigor of the conclusion, no matter whether the technique was traditional or modern.

O. D. Creutzfeldt

(Words of Welcome, Seventh European Neuroscience Congress, Hamburg, 1983)

\section{Introduction: The Divided Outer World}

Basic sensory functions depend on physical stimulus characteristics, physiological perceptual mechanisms, the state of the nervous system at the time of stimulus arrival, and also on the exact location of the stimulus on the receptor plane. In the somatosensory system, for example, the spatial resolution for mechanical stimuli differs according to whether they are applied to the fingertips or to the arm area, and the visual system shows pronounced functional differences between the center and the periphery of the retina, related to the different local packing densities of the photoreceptors and their different connections to the retinal ganglion cells.

The density of peripheral receptor and nerve cells has a direct relation to the cortical representation in a given sensory modality. In the somatosensory system a distorted representation of the body surface results in the homunculus, where relatively large cortical areas are devoted to small portions of the body like the tongue or the fingers, while other, larger areas of the body surface have only small 
cortical representations. In a similar way, the retinotopic projection of the visual field onto the visual cortex appears to be weighted by its functional importance and the anatomical wiring in the retina. Due to the cortical magnification factor (Whitteridge 1973), the central areas of the retina have a much larger cortical representation than the corresponding peripheral areas (Holmes 1945). Information processing in the sensory systems of the brain is performed in a complex manner, partly sequential, partly parallel. Differences in processing of incoming information depend not only on physical stimulus attributes but also on the external location of the stimulus to the right or to the left of the subjective vertical. Because of the basic wiring of most mammalian. nervous systems, including man's, primary sensory information of all modalities is directed to either the left or right hemisphere of the cerebral cortex. Although there exists some overlap, in general, stimuli which appear on the left of the subjective vertical are projected to the right cerebral hemisphere while stimuli on the right are projected to the left hemisphere. Similarly, the motor system is dominated by contralateral connections, and for more specialized purposes like writing or tool handling one hand is sufficient and one side of the brain may come to be preferred. The question of how the brain achieves its integrative task of uniting the halves of the perceptual world and enabling coordinated motor activity of twin organs has prompted much research in the neurosciences.

Since Broca's (1861) and Wernicke's (1874) work on aphasia, functional differences in language abilities between the two hemispheres have been known. This led to the concept of lateralized speech centers in the brain. Starting with Sperry's experiments on "split-brain" patients (Sperry 1964, 1968), other differences in general information-processing strategies which cannot simply be attributed to different language capabilities of the left and right halves of the brain were established, and a vast scientific literature has since accumulated (e. g., Gazzaniga 1970; Dimond and Beaumont 1974). The basic concept that the left hemisphere is superior in processing sequential, abstract input, while the right hemisphere is better in dealing with holistic, gestalt-type information processing, including the recognition of faces or spatial orientation (cf Jeeves 1984; Grüsser 1984), has now attained general acceptance. These results are based on experimental work in various sensory modalities.

In the visual system, functional differences between different regions of the visual field cannot be discussed simply in terms of a left/right distinction, because the left visual field projects onto the nasal retina of the left eye but onto the temporal parts of the right eye, whereas stimuli in the right visual field are processed by the nasal retina of the right eye and the temporal retina of the left eye. Anatomical and physiological left/right differences in the visual system can be located only beyond the optic chiasm, where the nerve fibers originating in the nasal retina cross to the contralateral cerebral hemisphere. The functional regional differences which occur in visual acuity (Wertheim 1894; see Sects. 5.2 and 6.2.2.3) are attributed to the different densities of photoreceptor distribution in the retina and appear also to be related to the relative importance of the occurrence of visual stimuli in the center or the periphery of the visual field. In addition, the regional distribution of temporal sensitivity of the visual system shows a systematic difference between the nasal and temporal parts of the retina which does not simply reflect general differences between the right and left visual fields or between the functional capability of the left and right cerebral hemispheres (see Sects. 5.1 and 6.2.2.2). 
Eye movement recordings during fixation suggest that the vertical meridian also plays some functional role in vision. Constant movement of the image on the retina is an important prerequisite for normal visual function. Human psychophysical data suggest that eye movements are related to the fact that high spatial sensitivity of the retina is confined to a very small area of about $20^{\prime}$ arc diameter in the center of the fovea (Alpern 1972). The perception of a visual scene is reconstructed from a sequence of sensory data obtained by scanning over different positions in the visual field. In addition, continuous eye movements are important for perception, since stabilized retinal images result in the disappearance of the visual percepts (Yarbus 1967; Ditchburn 1973). During visual fixation of an object, drifts and saccades occur with a directional specificity: Nachmias (1959) found that $63 \%$ of all saccades go off at between $50^{\circ}$ and $90^{\circ}$ (i. e., toward the upper octant of the visual field). A bivariate normal distribution of eye movement directions was computed which was tilted toward the vertical meridian (see Fig. 1A). The results of Bennet-Clark (1964) and of Yarbus (1967) on eye movements during continuous fixation of a visual target are in agreement, as illustrated in Fig. 1B and C: The eye fixation fields are not restricted to horizontal movements but contain a pronounced vertical component.

This suggests that the vertical direction is also important for visual perception. The results which I will discuss show that functional regional differences in the visual system are not confined to the horizontal meridian reflecting cerebral hemispheric specialization in terms of a left/right distinction; there exist, in addition, related differences between retinal areas revealed by electrophysiological and psychophysical experiments. These data suggest that different characteristics of the perceptual mechanisms are activated by stimuli in the upper and lower halves of the visual world. I will thus not consider the well-known, widely propagated, and presently popularly accepted differences in higher information processing presumably located in the two halves of the cerebral cortex, but rather review anatomical, physiological, and functional differences between the upper and the lower hemiretinal systems.

Some of the earliest quantitative experimental work on visual perception dealt with visual acuity. Aubert and Förster (1857) and Landolt and Hummelsheim (1904) investigated the regional differences in spatial visual resolution, and their results showed that visual acuity is higher for upper than for lower retinal stimuli. This suggests a fundamental functional system difference (see also Sect. 6). In the course of electrophysiological studies in man (Eason et al. 1967; Lehmann et al. 1977; Lehmann and Skrandies 1979) it has been observed that stimulation of upper retinal areas evokes electrical brain activity with shorter component latencies than stimulation of lower retinal areas. These findings lead to the question of whether the functional (visual acuity) difference is related to the electrophysiological difference reflected in component latency. In addition, it appears of interest to investigate whether only some isolated visual functions differ in the upper and lower visual fields, or whether there is a global distinction in visual processing capability.

Various aspects of perceptual efficiency can be examined psychophysically, as illustrated in Sect. 5, while electrophysiological methods offer the possibility of analyzing retinal and cortical activity independently. Such electrophysiological data will help to establish at what stage of the visual system the difference in per- 

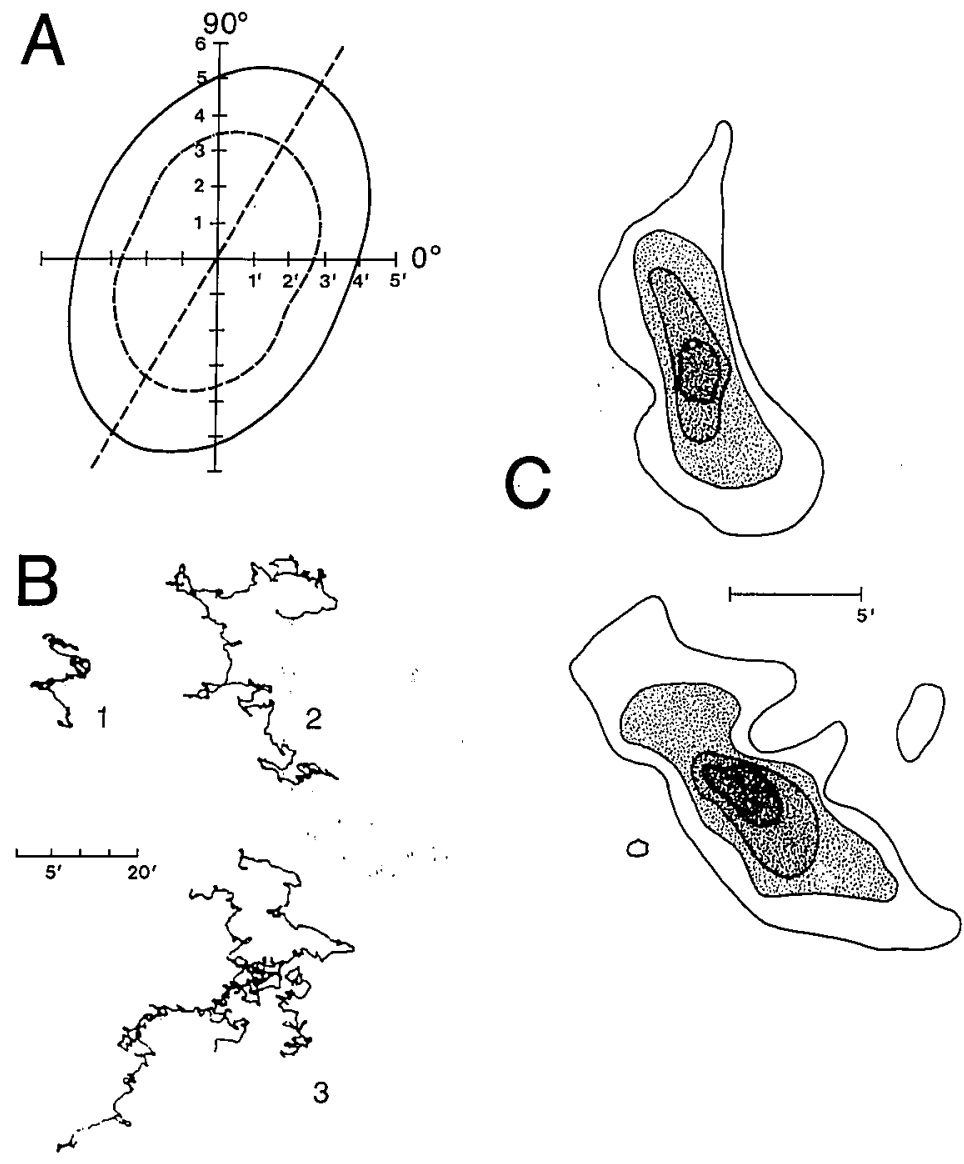

Fig. 1A-C. Distribution of eye movements during fixation of a steady point. A Direction of the visual axis, showing an approximately bivariate distribution. Any point of the retinal image will lie in the region of the solid curve $68 \%$ of the time during a fixation interval of $30 \mathrm{~s}$. (Data from Nachmias 1959, Fig. 6; redrawn after Ditchburn 1973) B Eye movements during fixation for $1.10 \mathrm{~s}, 2.30 \mathrm{~s}$, or $3.60 \mathrm{~s}$. (Redrawn after Yarbus 1967, Fig. 54) C Contour maps of the direction of the visual axis for two subjects showing the area scanned during a fixation period of $10 \mathrm{~s} .100 \%$ of all eye movements lie within the outermost border of the fixation fields; $25 \%$ lie within the innermost contour (Redrawn after Bennet-Clark 1964, Figs. 5 and 6)

formance is initiated. Differences in the processing of the upper and lower retinal visual stimuli could be explained by a general superiority of the upper over the lower hemiretinal system or alternatively by local physiological differences. Evoked potential data are obtained only at one of the last steps in the chain of visual information processing, and thus must be complemented by the analysis of activity at earlier stages in the visual system in order to localize the origin of the functional differences.

The correct interpretation of evoked potential data depends on the use of unambiguous recording and data analysis techniques in order that we may arrive at 
physiologically meaningful conclusions from scalp-recorded electrical brain activity. Such methods have been developed as topographical techniques during recent years (Lehmann 1977; Lehmann and Skrandies 1980, 1984; Skrandies and Lehmann 1982a, b), and these techniques have been used successfully to study electrophysiological differences between the upper and lower hemiretinal systems in man, as illustrated in Sect. 3.

I will first discuss how scalp-recorded brain activity can be used to gain insight into complex electrical brain mechanisms in spite of some inherent shortcomings of the "indirect" recording method which is the only possible one in intact human subjects (Sect. 2). It will be shown that evoked potential recordings in man may yield information which complements the results of "direct" intracranial recordings in animals. Evoked potential data reflect the mass activity of large neuronal populations, and thus allow conclusions on global functional differences of larger brain areas which may be missed in selective single unit recordings (see Sect. 2.2). Various experiments which have been performed comparing the upper and lower retinal systems in man will be described below. First, I will report on electrophysiological differences in evoked potentials between upper and lower hemiretinal stimulation obtained in different subject populations under various stimulus conditions (Sect. 3). The implications of the results for the clinical application of evoked potentials will be demonstrated in Sect. 3.3. Then electrophysiological investigations of the different stages of the human visual system will be described (Sect. 4), and an attempt will be made to relate the electrophysiological findings (which are usually discussed only in formal terms of amplitudes, latencies, or scalp topography) to functional differences between the upper and lower hemiretinal systems. Prompted by the electrophysiological results, a series of studies has been made investigating visual functions such as temporal sensitivity, contrast sensitivity, and discrimination of meaningful stimuli (Sect. 5). These functional aspects appear of major importance, since even the most significant finding is not of much interest if its interpretation remains restricted to a small and highly specialized field of research and is not paralleled by behavioral concomitants.

Evidence on differences between the upper and lower retinal systems is scattered through the literature, and in most cases differences between upper and lower retinal areas have not been studied systematically. A review of the pertinent data from neuroanatomy, physiology, psychophysics, psychology, and ophthalmology will be given in Sect. 6 .

\section{Analysis and Origin of Visual Evoked Potential Components ${ }^{1}$}

\subsection{Topographical Analysis of Scalp-Recorded Brain Activity}

In the early 1870 s the English physiologist $R$. Caton started to investigate the electrical activity of the brain, and in 1875 he presented his results obtained in rabbits and monkeys to the British Medical Association. From these experiments it

\footnotetext{
${ }^{1}$ A related short account of some of these ideas illustrating different sets of data has been given (Skrandies 1986a) elsewhere
} 
became clear that electrical brain activity can be divided into spontaneous activity and evoked activity: "In every brain hitherto examined, the galvanometer has indicated the existence of electric currents. ... Impressions through the senses were found to influence the currents of certain areas ..." (Caton 1875). The same is true for the electrical activity of neural populations in the human central nervous system which can be recorded from the intact scalp of man: the electroencephalogram (EEG) reflects spontaneous activity, as first discovered by Berger (1929), while evoked potentials are in general elicited by adequate stimulation of sensory organs.

Evoked brain potentials reflect the synchronous activity of neuronal populations whose geometry in the brain produces an electrical field which can be recorded from scalp electrodes. Because of volume conduction this electrical field can be detected over the entire scalp, and conventional recordings of potential differences between two electrodes reflect only the potential gradient along the line between the recording sites. As will be shown in this section, the analysis of such potential waveshapes should be replaced by an adequate quantitative treatment of the electrical data as scalp field distributions. Recording of evoked potentials is a noninvasive method with high time resolution (down to milliseconds) and high sensitivity to spontaneous and induced changes of the state of the brain. Although the mechanisms of the electrogenesis of scalp-recorded brain activity are not clear in detail, studying its covariation under experimental conditions appears fruitful. A high correlation between the firing probability of single cortical cells and the evoked potential recorded near the cell body was reported by Fox and O'Brien (1965). Creutzfeldt and Kuhnt (1967) concluded from their studies on cats that evoked potentials are composed in inhibitory and excitatory postsynaptic potentials as well as of synchronous fiber activity. Thus, a component of the evoked potential may reasonably be defined as the occurrence of synchronous activation of a large neural population. Due to the fact that the human cortex is strongly folded, electrical fields generated by oppositely oriented neuronal generators will cancel. In spite of this problem in all sensory modalities, there are scalp-recorded components that are very consistent across subjects. An evoked component may be recorded from the intact human scalp:

1. If it has a fixed temporal relation to the occurrence of a physical stimulus or to some internal stimulus-processing mechanisms [which elicits a so-called endogenous component; see Donchin et al. (1978) and Skrandies et al. (1984)]

2. If there is a population of neurons in the brain which is activated synchronously

3. If the neuronal structures involved produce an open electrical field as a net response

\subsubsection{Why Topography?}

Topographical analysis of EEG and evoked potential data is in general considered a tool to define the intracranial sources of scalp-recorded electrical brain activity. The methods of localizing the sources of electrical activity are based on the work of von Helmholtz (1853). It is a physical fact that the scalp potential distribution 
can be calculated when the instantaneous configuration, location, and orientation of the activated intracranial neural populations are known [the so-called forward problem; cf Wilson and Baley (1950)]. But it is important to keep in mind that the reverse is not true: scalp field configurations do not allow unequivocal predictions of the underlying intracranial sources. Attempts to solve this "inverse"problem, i. e., how to calculate the sources of potentials in a conductive medium when the potential field on the surface is given, have no clear solution. Therefore, computations to localize model dipoles always have to rest on certain assumptions regarding conductivity, homogeneity, and geometry of intracranial media, and assumptions concerning the number, location, and extension of electrical model dipoles must also be made in order to obtain physiologically meaningful interpretations (Schneider 1972, 1974; Kavanagh et al. 1978; Sidman et al. 1978). Because of this "inverse" problem, and because of the fact that millions of individual neural generators are active at any given moment, the goal of intracranial generator localization presently appears of less practical value than claimed. This chapter will discuss a different approach to the treatment of topographical data. I will show that scalp distributions are being investigated primarily not in order to identify intracranial sources of evoked brain activity, but because the scalp topography of the electrical fields of the brain characterizes brain electrical events unambiguously in terms of latency and scalp location. In addition, topographical data provide a basis for testing hypotheses concerning the processing mechanisms of different aspects of sensory information by the central nervous system. Irrespective of whether the exact intracranial sources of the scalp-recorded brain activity are known, the interpretation of scalp distribution data, combined with knowledge of the anatomy and physiology of sensory brain systems, can lead to physiologically meaningful conclusions. Comparison of scalp distributions evoked under different stimulus conditions gives direct information on the identity (or nonidentity) of the neural populations activated under these conditions. While identical distributions may or may not be generated by different neural populations, nonidentical scalp fields must be caused by different neural generator mechanisms. In the present section I will discuss how scalp-recorded brain activity can be used to investigate complex electrical brain mechanisms independent of the basic shortcomings of the indirect (i. e., extracranial) recording method which is in general the only one possible in human subjects.

Section 2.1.2 will show how quantitative measures of electrical brain activity are extracted without the choice of a reference electrode. Subsequent sections will illustrate applications of these techniques to physiological questions concerning the differences between upper and lower retinal areas, which are revealed by evoked potential recordings under various stimulus conditions.

\subsubsection{Some Basic Considerations}

Evoked potential data is commonly analyzed in terms of components which are believed to be subsets of electrical brain activity related to different neural processing mechanisms. The prime aim of evoked potential studies is the establishment of relations between such components and experimental or physiological parameters. The problem of adequate definition of components has been exten- 
sively discussed in the literature. Techniques ranging from simple peak measurement to elaborate multivariate statistics have been proposed, and the relationship to underlying neuroanatomical structures has also been stressed:

The definition of the components is an empirical event and their localization to sets of neurones requires a deep knowledge of the structure and dynamics of the region in which the event occurs, to the extent that the locations, patterns of spread, and time courses can be predicted for particular components. ... It is, to be sure, a long way from the olfactory bulb to the association neocortex, but I will maintain that unless you develop and use a theoretical basis for predicting waveforms from local neural anatomy, topology, and dynamics, you will be wallowing in principal components indefinitely (Freeman 1978, p. 507).

One should add that evoked potential analysis must not only consider the underlying neural structures, but has first of all to acknowledge that we are dealing not with sets of potential waveshapes but with electrical fields generated by the brain. Much controversy regarding evoked potential data originates from the ambiguous interpretation of waveshapes. Looking at potential fields can prevent one from "wallowing in components," and in the following sections I will illustrate quantitative methods which analyze evoked potential data adequately as electrical fields.

Scalp-recorded electrical brain activity reflects the mass response of large neural populations which spreads throughout the brain via volume conduction. This is illustrated by the auditory brain stem response: auditory stimuli are followed by potential components of very short latency (less than $10 \mathrm{~ms}$ ) which can be recorded from the intact scalp of man, and there is direct evidence that these components reflect the electrical activity at the early processing stages of the auditory pathway at the level of the brain stem (Jewett et al. 1970; Starr and Achor 1975). Field potentials generated by the activation of optic nerve and optic tract fibers in the cat visual system can be recorded in widespread regions of the brain (Skrandies et al. 1978). Detailed analysis of field potentials in the retina is possible only in animal experiments by means of direct intraocular recording of mass activity and single unit responses (Fig. 2A). The mass activity of the $\mathrm{Y}$, $\mathrm{X}$-, and $\mathrm{W}$ ganglion cells of the cat retina following electrical stimulation of the optic tract may be discriminated at the optic disc (Fig. 2B), and the field potentials "stray" from the retina into the vitreous body of the eye. The field potential components persist even when the electrode is several millimeters in front of the retinal surface (Fig. 2C-E). In addition, a far-field potential generated by the Y-fibers entering the eye can still be seen over the temporal retina at $6 \mathrm{~mm}$ eccentricity (Fig. 2C). Similarly, the early component of the Y-fibers can be recorded in many brain regions that have no direct anatomical connection to the optic nerve or tract (Skrandies et al. 1978), and these data show that potential components recorded at a given point in the nervous system need not be generated at that site. Thus, any statement about the location of neural generator populations of components of evoked brain activity requires a solid experimental and physiological basis. In the field of EEG the problem of the position of the reference electrode is considered crucial, and this topic has received much discussion in the literature. I will show in this section how the problem can be avoided and how surface-recorded EEG data can be interpreted unambiguously independent of the location of the reference electrode. 


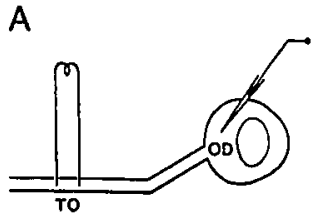

B

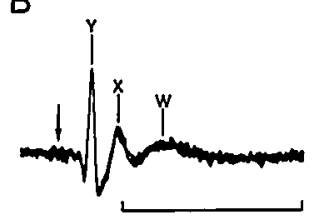

C

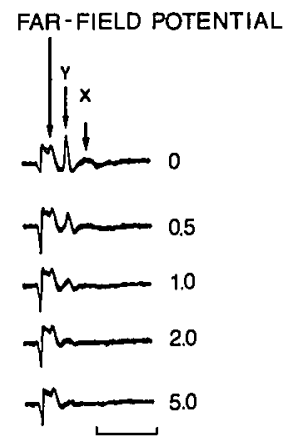

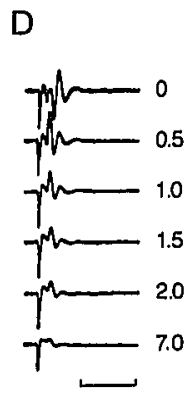

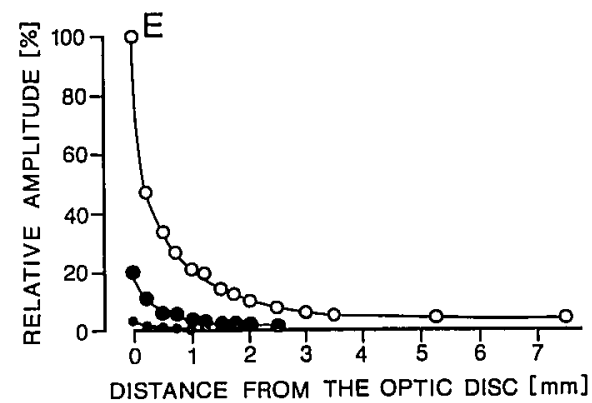

Fig. 2. A Experimental setup for the study of field potentials in the cat eye. Stimulating electrodes are stereotactically placed in both optic tracts (Horsley-Clarke coordinates: anterior +10 , lateral 7). Single unit responses and field potentials were recorded from retinal ganglion cells. $O D$, optic disc; TO optic tract. Further details are described by Skrandies et al (1978) B Antidromic field potentials recorded at the optic disc after antidromic electrical stimulation of the optic tract. Three components can be identified as the mass responses of Y-, X-, and W-ganglion cells. Arrow indicates stimulus onset. C Field potentials over the temporal retina at $6 \mathrm{~mm}$ eccentricity after electrical stimulation of the optic tract recorded at various distances from the retina. Numbers indicate distance to the retinal surface in millimeters, and the amplitudes of the locally generated potential components decrease gradually while the amplitude of the far-field potential originating from the optic disc remains constant. The first sharp peak in the waveforms is the electrical stimulus artifact corresponding to the instant of stimulation. D Field potential components recorded over the optic disc when the recording electrode is pulled back from the retina. Numbers indicate distance to the retinal surface in millimeters. The first sharp peak in the waveforms is the electrical stimulus artifact corresponding to the instant of stimulation. E Amplitudes of field potential components over the optic disc as a function of distance to the retina. The three ganglion cell classes show a different amount of amplitude decrease. Dots, W-field potential; closed circles, X-field potential; open circles, Y-field potential. Time scales (bars) for all traces indicate $5 \mathrm{~ms}$. (Data from Skrandies et al. 1978)

Electrical brain activity is recorded as potential differences between recording points on the scalp, and accordingly the position of both the reference and the recording electrode influences the waveform pattern of electrical activity. The potential waveforms are always ambiguous in terms of amplitude and phase of electrical events as analyzed over time, but the characteristics of the underlying 
Fig. 3. Potential waveshapes evoked by a $30^{\prime}$ arc reversing checkerboard pattern presented with $62 \%$ contrast as a test field of $8.7^{\circ} \times 11.4^{\circ}$ arc to the upper hemiretina. Arrowheads and numbers indicate peak latencies (in $\mathrm{ms}$ ) of the corresponding waveshape. The electrode array starts at the vertex and extends in regular steps to $2 \mathrm{~cm}$ below the inion (see head schema on the right). Data are referred to the average reference

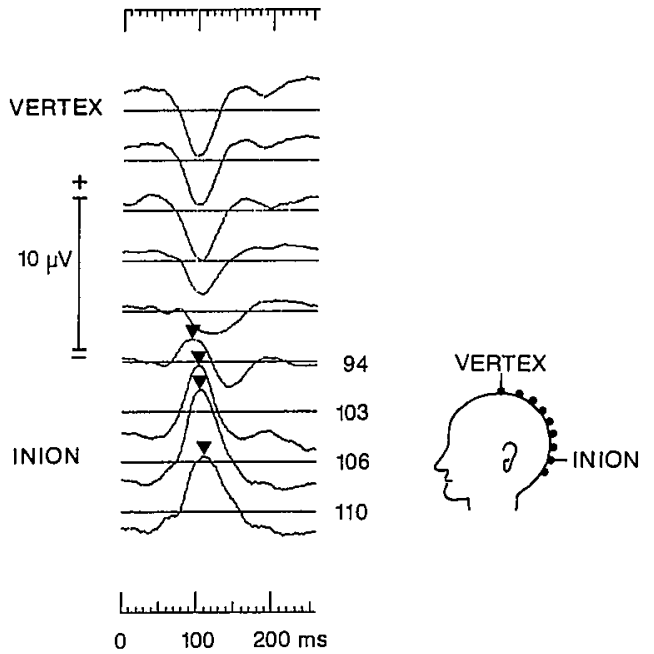

electrical fields of the brain (and thus of the scalp fields) are constant and reference-independent at each moment in time. Accordingly, only electrical fields may be interpreted in a meaningful way (Lehmann 1977; Nunez 1981). Strict topographical analysis strategies must be applied to electrical scalp fields in order to avoid the problem of the reference electrode and ambiguous data interpretation (Lehmann and Skrandies 1980; Skrandies and Lehmann 1982a).

The inspection of evoked potential waveshapes may yield confusing results. Visual evoked potentials (VEPs) recorded from nine midline electrodes evoked by a checkerboard reversal stimulus presented to the upper hemiretina are shown in Fig. 3. These waveshapes are referred to the "average reference", i. e., to the mean potential of all electrodes in the field. Note that the major occipitally positive component shows different peak latencies in different recording channels, as indicated by the arrowheads in Fig. 3 (e. g., channel 6 has a peak latency of $94 \mathrm{~ms}$; channel 7, $103 \mathrm{~ms}$; channel 8,106 ms; channel 9,110 ms). These systematic changes in latency might be interpreted as evoked activity moving from anterior to posterior brain areas. A change of the location of the reference electrode makes the picture more complex. The identical data set recomputed against other reference sites is illustrated in Fig. 4A: On the left, the data of Fig. 3 are referred to the vertex reference, in the center an electrode $2.5 \mathrm{~cm}$ posterior to the vertex is used as reference, and on the right an electrode $5 \mathrm{~cm}$ posterior to the vertex is used as reference point. Latency differences occur in different recording channels, and there appear changes in latency depending on the reference chosen: for example, channel 8 has peak latencies of 103,105 or $106 \mathrm{~ms}$. The complete possible data set consists of the potential waveshapes referred to every other electrode of the array (i. e., for nine electrodes 72 different waveshapes are possible). All these waveshapes are illustrated in Fig. 4A-C. Evidently, this rather simple set of data presents an astonishing amount of information and confusion. It is important to keep in mind that we are dealing with identical data (the same data set has been recomputed) and that all statements about peak latencies are correct. There is no good reason to prefer one set of waveshapes shown in Fig. 4 over the others. This 


\section{W. Skrandies}
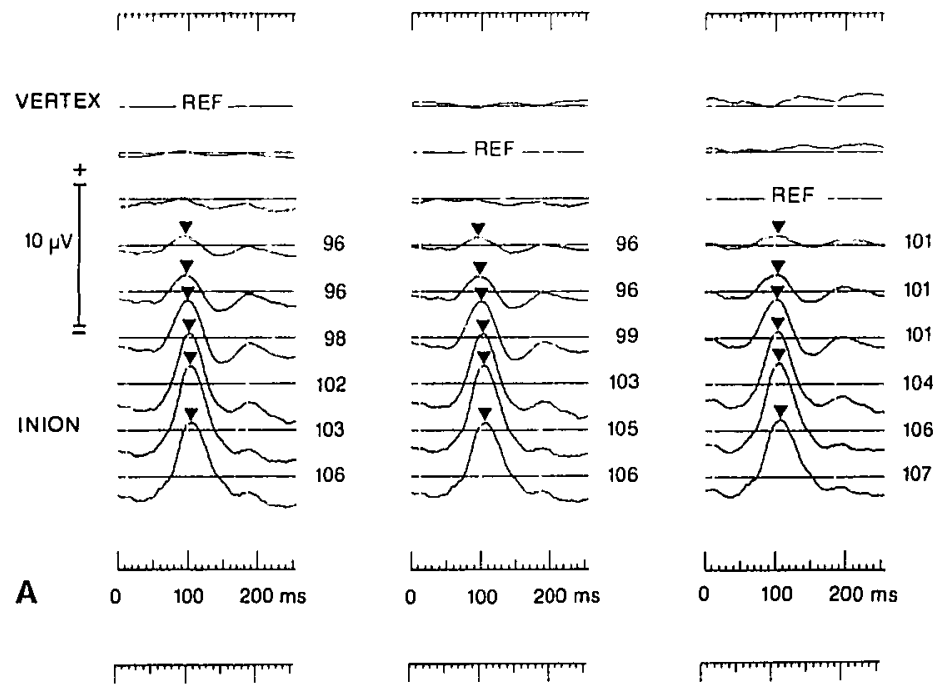

$$
\text { لمسلسلسيسلسساس } 200 \text { ms }
$$
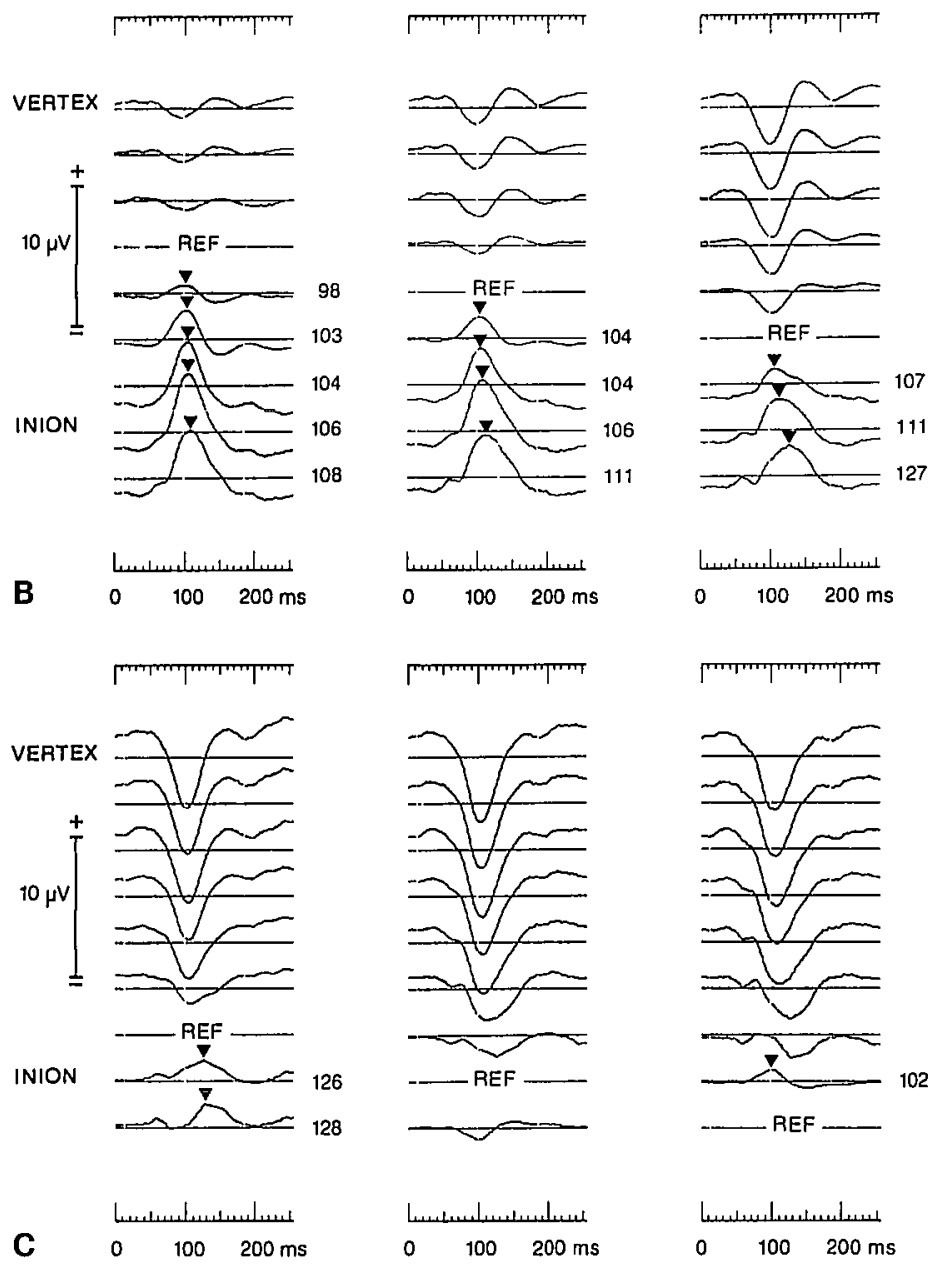

Fig. 4A-C. Data of Fig. 3 computed vs all possible reference sites. Each column shows the waveshape patterns when a given electrode is used as the reference. The reference channel $(R E F)$ shows a flat line. First electrode at the vertex $(\mathrm{Cz})$, last electrode $2 \mathrm{~cm}$ below the inion. Consult head schema in Fig. 3 for electrode locations. Arrowheads and numbers indicate peak latencies (in $\mathrm{ms}$ ) of the corresponding waveshape 

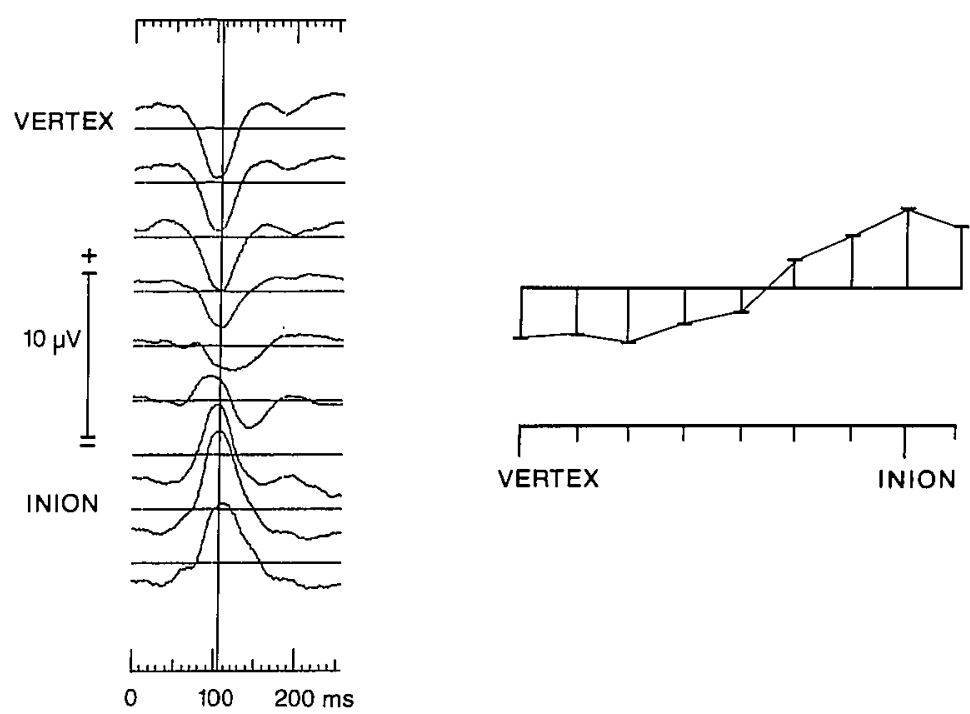

Fig. 5. Construction of a topographical potential profile at $106 \mathrm{~ms}$ latency. Amplitudes measured in each channel are plotted as a function of electrode position. The zero line corresponds to the value of the average reference. Changing the reference site shifts the zero baseline up or down, but leaves the shape (i.e., the location of maxima and minima, and the gradients) of the profile unchanged

dilemma of waveshape variability and ambiguous data intepretation can be overcome only by an adequate analysis technique which is independent of the reference electrode.

In contrast to what is suggested by Figs. 3 and 4, electrical brain activity does not move over larger distances on the scalp and different component latencies in different recording channels are not observed. This becomes evident when the data are treated as an electrical field, and Fig. 5 displays the data at $106 \mathrm{~ms}$ latency as a potential profile. This is simply a different arrangement of the data: the amplitude values at $106 \mathrm{~ms}$ are measured in each channel, and are entered at their respective electrode positions (Fig. 5 , right) resulting in a potential profile at the respective instant. When using two-dimensional electrode arrays, potential maps are the adequate topographical display of the electrical brain activity (e. g., Figs. $10,23,25)$. The major feature of the profile in Fig. 5 is an occipital positive maximum surrounded by steep gradients, while over anterior areas a flat distribution prevails. Most importantly, a change of the reference point will not change the shape of the potential profile, since a different reference would simply change the position of the zero potential level and thus the labeling of the potential values, while the potential maxima and minima and gradients within the field remained unaffected.

The information of Fig. 3 is topographically displayed in Fig. 6 as a series of potential profiles between 4 and $256 \mathrm{~ms}$ latency. This display contains the same amount of information as the waveshapes but stresses the topographical aspect of the data. 


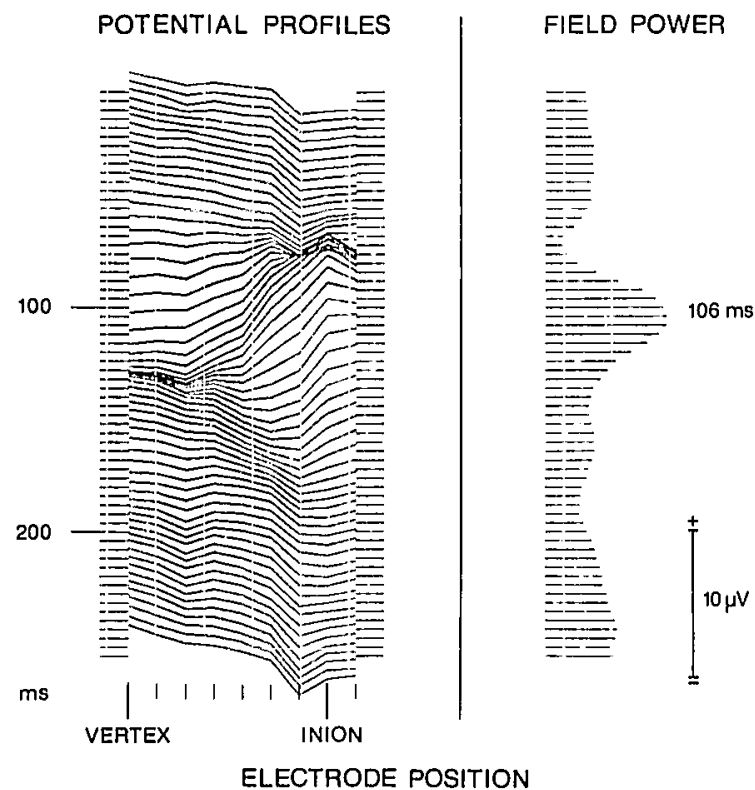

Fig. 6. Series of 64 consecutive potential profiles between 4 and $256 \mathrm{~ms}$ (left), and global field power (right) computed every $4 \mathrm{~ms}$ (same data as Figs. 3 and 4). The time axis runs from top to bottom, the anterior-posterior electrode positions from left to right. Positive potential values upward

It appears reasonable to define the latency of an evoked component as the occurrence time of maximal activity in the scalp field reflecting the time of synchronous activation of a maximal number of neurons. In order to quantify the electrical activity of each scalp field, we have proposed a measure of global "field power" that is computed as the root mean square of all possible potential differences in the field, or a related measure of "potential range" (i. e., the difference between the maximal and minimal potential values obtained, irrespective of their scalp location) which yields similar results (Lehmann and Skrandies 1980; Skrandies and Lehmann 1982a). These measures quantitatively determine the amount of electrical activity at every instant independent of the reference electrode, and pronounced scalp fields with high peaks and troughs and steep gradients will result in high field power. Times of maximal field power define the occurrence of a component. In Fig. 6 global field power is plotted on the right side as a function of time adjacent to the corresponding potential profiles. The field power is low at early latency times but increases around $100 \mathrm{~ms}$, reaching a maximum at $106 \mathrm{~ms}$. This instant defines the latency of the evoked component. Afterward, field power decreases again (see Fig. 6).

Topographical changes over time can be analyzed in detail by plotting the potential profiles at successive points in time, as illustrated in Fig. 7. Contrary to what was suggested by the potential waveshapes in Fig. 3, it is obvious that there is no change in the scalp location of the occipital component, because between 90 and $120 \mathrm{~ms}$ the peak remains at the same electrode and only the potential gradients change.

After determining component latencies, the next step is the analysis of the scalp field features at these latency points in terms of scalp locations of the maxima, minima, and potential gradients. These derived measures are by definition reference-independent (i. e., the location of a maximum or a minimum does not 
Fig. 7. Enlarged display of part of the profile series of Fig. 6, between 90 and $120 \mathrm{~ms}$. Note that there is no change in component location as was suggested by the potential waveshape pattern of the same data in Fig. 3

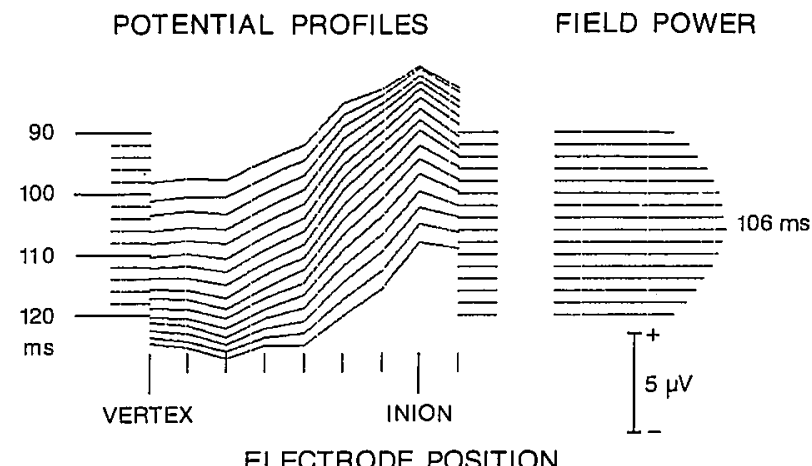

ELECTRODE POSITION

change when the reference is changed). Different subject populations or different experimental conditions may then be compared statistically using these extracted values.

This topographical analysis strategy defines evoked components independent of the reference point, and treats electrical scalp field data unambiguously. Thus, scalp field data can be related to intracranial mechanisms in a physiologically meaningful way. This methodological approach is used in the present text to analyze scalp-recorded electrical data.

\subsection{Origin of Visual Evoked Potential Components}

In most visual evoked potential (VEP) studies the P100 component-an occipitally positive component of approximately $100 \mathrm{~ms}$ latency-is evaluated, since it is very reliable and shows low intersubject variability. On the basis of its long latency and its scalp distribution elicited by lateralized half-field stimulation (Skrandies and Lehmann 1982b), this component is presumably of cortical origin. Further support for a cortical origin of the P100 component was obtained by analyzing components evoked by patterns with contrast borders and comparable stereoscopic checkerboard patterns which depend on binocular fusion in the visual cortex.

A prerequisite for the analysis of the origin of VEP components is knowledge of the underlying neural processing steps in the visual system. Dynamic random dot stereograms (RDS) which do not contain monocular cues evoke potentials of cortical origin (Lehmann and Julesz 1978), since in the human visual system the input of the two eyes remains separated until the level of the visual cortex. The binocular interactions crucial for stereoscopic depth perception must be cortical functions. Thus, the "imaginary cyclopean eye" (von Helmholtz 1910; English edition Southall 1962) or the "cyclopean retina" (Julesz 1971) are operationalized in the visual cortex. Purely cortical neuronal generating mechanisms were studied by recording the brain's electrical activity evoked by dynamic RDS stimuli, skipping operationally all processing stages prior to the activation of cortical binocular disparity neurons. The comparison of a stereoscopic stimulus condition with a similar two-dimensional stimulus condition gives information on the similarity of the neural processing of these two classes of stimuli (Skrandies and Vomberg 1985). 
In 15 subjects evoked potentials were recorded from a midline row of nine electrodes equidistantly spaced between the vertex and $5 \mathrm{~mm}$ above the inion (see inset in Fig. 8). Stereoscopic checkerboard reversal patterns composed of random dots were shown to each eye separately every $20 \mathrm{~ms}$ using a special visual pattern generator (for details see Skrandies and Vomberg 1985). This stereoscopic checkerboard was reversed every $256 \mathrm{~ms}$, and the change served as the stereoscopic stimulus while monocular processing stages received only dynamic noise.

In order to assure that the RDS stimulus did not contain monocular cues, evoked brain activity was also measured with monocular stimulation. In this conditon evoked potentials did not exceed the noise level. This is in line with earlier findings that in stereoblind patients no stereoscopically evoked brain activity can be recorded (Vomberg and Skrandies 1985). Two-dimensional black and white checkerboard reversal stimuli presented binocularly with the same mean luminance and high contrast were used for comparison. Potential profiles were analyzed, component latency was determined by the occurrence of the maximal field power computed in a time window between 80 and $160 \mathrm{~ms}$, and component locations were defined as the electrode position of maximal voltage at this latency (see Sect. 2.1).

A

\section{STEREO}

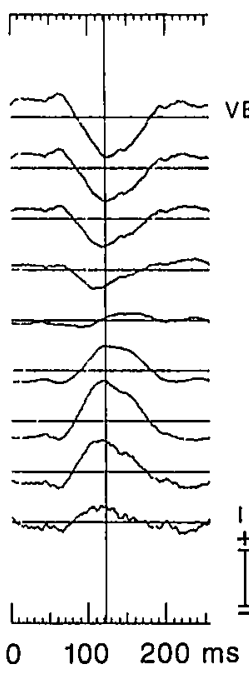

\section{BINOCULAR}

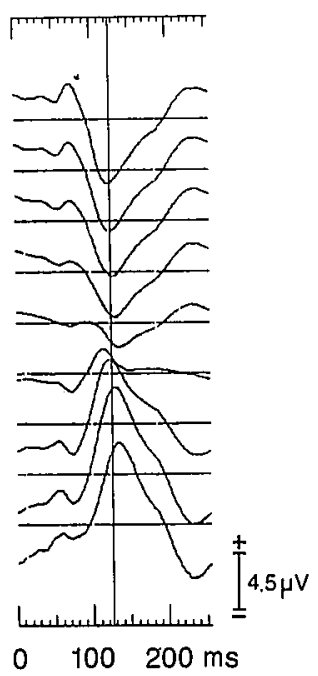

B
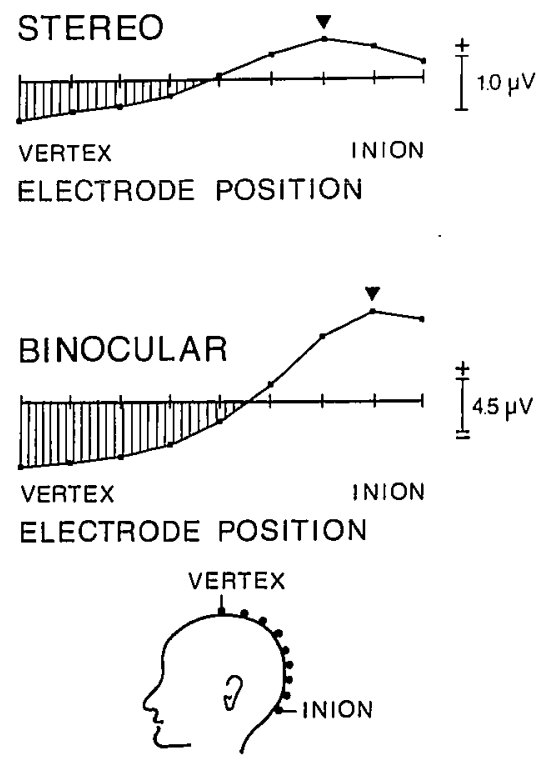

Fig. 8. A Grand mean potential waveshapes of 15 subjects evoked by stereoscopic stimuli or comparable binocular contrast stimuli. Data were recorded from a midline row of nine electrodes equidistantly spaced between the vertex and $5 \mathrm{~mm}$ above the inion, and are referred to the average reference. The occipitally positive component which was determined by the occurrence of maximal field power is marked by a vertical line. $\mathbf{B}$ Grand mean potential profiles at component latencies. The data of $\mathbf{A}$ are displayed as scalp field distributions. The location of the maximal positivity is marked by arrowheads. Note larger amplitudes of components evoked by contrast stimuli and the more anteriorly located potential maximum of the stereoscopically evoked component. Head schema shows electrode positions 
The configuration of the potential waveshapes was similar for stereoscopic and binocular contrast stimuli: In both stimulus conditions an occipital positivity occurs about $120 \mathrm{~ms}$ after the stimulus (Fig. 8A). In the subject population component latencies were not different, whereas amplitudes of the RDS-evoked components were significantly smaller than those of the binocular contrast VEPs. Figure 8B shows the potential profiles at their respective component latencies. For both stimulus conditions a positive, occipital maximum is seen. This maximum was located more anteriorly for stereoscopic stimuli (mean $1.8 \mathrm{~cm}$ ) in the subject population, and the difference in component location was statistically significant $(t=3.59, p<0.0025)$.

Smaller amplitudes of the stereoscopically evoked components suggest that fewer neurons are activated synchronously by this stimulus, while the topographical differences prove that differently located or oriented neural generator populations are activated by three-dimensional stimuli.

These results are in line with single unit recordings in the visual cortex of monkeys, where depth-sensitive neurons were found (Poggio and Fischer 1977). Hubel and Wiesel (1970a) and von der Heydt et al. (1981) claimed that area 18 (which in man lies anterior to the primary visual area 17) contains more neurons responding to disparate stimuli. In single unit studies, sampling biases may mimic a differential distribution of functional units but evoked components reflect the mass activity of neural populations and global functional differences may be found. A more anteriorly located potential peak evoked by RDS stimuli in man, is a strong indication that stereoscopic stimuli activate more neurons in area 18 than in the primary visual cortex. The potential components evoked by contrast changes had the same latency (and a similar but nonidentical topography) as the stereoscopic components which are definitively of cortical origin. These findings thus strongly suggest that the $\mathrm{P} 100$ component evoked by two-dimensional contrast stimuli is of cortical origin.

\subsection{Temporal Activity Patterns}

Activity patterns of longer duration can be investigated topographically by plotting the most distinct features of the scalp fields (e. g., the locations of the potential maxima and minima) as a function of time. This yields information on the similarity of neural activation cycles evoked in different experimental conditions. The topographical differences between stereoscopic and contrast stimuli described in Sect. 2.2 are not restricted to the occurrence times of components: Fig. 9 shows that the differences prevail over long durations of time. The location means of 15 subjects are plotted at 5-ms intervals, and significant differences between stereoscopic and contrast evoked activity were established by computing paired t-tests as an exploratory statistic. The data of Fig. 9 suggest that the time course of the activation patterns in the two stimulus conditions is similar but that their topography is different. Both potential maxima and minima have different locations over longer epochs, indicating sustained differences in the activation of nonidentical neural generator populations.

Such neurophysiological differences are in line with functional differences between electrical brain activity evoked by dynamic RDS stimuli or by stimuli with 

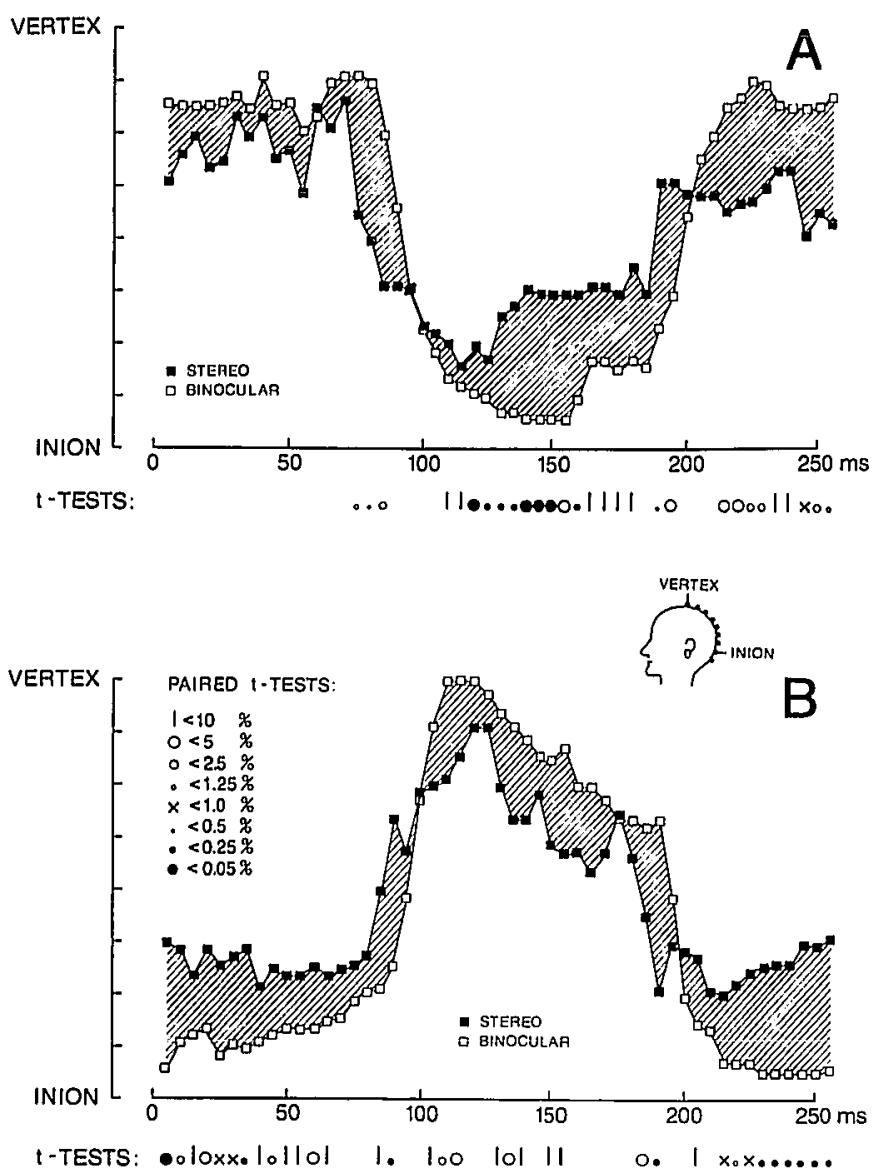

Fig. 9A, B. Mean location of potential maxima (A) and minima (B) of 15 subjects between 5 and $255 \mathrm{~ms}$ latency at intervals of $5 \mathrm{~ms}$ evoked by stereoscopic stimuli or by similar binocular contrast stimuli. Hatched areas indicate differences in location. The results of paired t-tests computed on the individual data are shown below each graph. Significant differences persist over longer time epochs. Note clusters of high significance levels

contrast borders. With temporally modulated patterns, Skrandies (1986b) showed that stereoscopic stimuli appear to persist visually at much lower time thresholds than contrast stimuli. In addition, with dynamic RDS stimuli a significant inverse linear relationship between temporal modulation frequency and evoked potential amplitude was found which was not observed with comparable contrast stimuli (Skrandies 1986b; Skrandies, in preparation).

\subsection{Conclusion}

The topographical analysis strategies outlined above are transparent and simple to apply, and they yield direct quantitative measures of component latencies and scalp locations. The derived measures are reference-independent and can be used 
for statistical comparisons between subject populations or between experimental conditions. Application of these topographical methods is not limited to potential fields; they have also been used successfully with multivariate statistical analysis: principal component loadings (Skrandies and Lehmann 1982b) and principal component scores (Skrandies 1983; Skrandies et al. 1984) have been topographically examined. The combination of multivariate statistics and scalp field distribution data proved a powerful tool in the analysis of differences in VEP lateralization (Skrandies and Lehmann 1982b) and of electrical brain activity recorded during the performance of a visual information-processing task (Skrandies 1983; Skrandies et al. 1984).

Other reference-independent descriptors of potential fields use information on the local gradients or on the curvature of the field distribution. The method of current source density analysis (Mitzdorf and Singer 1978; Mitzdorf 1985; McKay $1984 a, b)$ or the "source derivation" as it became known in EEG analysis (Hjorth 1975), examines the second derivative of the potential distribution with respect to space. The magnitude of the local curvature of the potential field is estimated at each instant and can be plotted as a function of time, similar to the convential potential waveshapes. Such measures were displayed as chronotopograms by Rémond et al. (1969) who illustrated the curvature of spontaneous alpha EEG potential fields. Some of our evoked potential data have also been analyzed by computing the current source density. As expected, this method did not yield conclusions basically different from those that had been derived from direct spatial analysis (see Sects. 3.2.1 and 3.2.2, Figs. 13, 14 and 21).

\section{Differences in Latency and Topography of Visual Evoked Potential Components}

In an earlier study using multichannel recordings of visual evoked brain activity, Lehmann and Skrandies (1979) found significant differences in component latency between the potential fields evoked by upper or lower hemiretinal stimuli with upper retinal stimuli yielding shorter latencies than stimulation of the corresponding lower retinal areas. In order to generalize and to extend these earlier findings on checkerboard reversal stimuli (Lehmann and Skrandies 1979) in this section I will report supporting multichannel data. Four different series of experiments were performed: Potentials evoked by checkerboard reversal stimuli were recorded in 45 channels and were topographically analyzed as complete scalp field maps (Sect. 3.1). Additional experiments using pattern reversal stimuli are described in Sect. 3.2.1. Findings on potential fields evoked by different presentation modes of an identical checkerboard stimulus will be reported in Sect. 3.2.2, and the results obtained with grating stimuli will be discussed in terms of component latency and scalp topography in Sect. 3.2.3. In all the experiments, consistent differences were found in scalp topography and in component latency between the electrical brain activity evoked by upper vs lower retinal stimuli. These data suggest that there are basic functional differences between the upper and the lower hemiretinal systems.

In a further study the practical implications of these findings for the clinical use of visual evoked potentials were investigated (Sect. 3.3). One application of evok- 
ed potential recording in the visual modality relies on the exact determination of component latencies in patients suspected to have multiple sclerosis (Halliday et al. 1973). Prolongation of component latencies by only a few milliseconds may classify a patient's result as pathological, and our data obtained in a population of unselected neurological patients show impressively how the physiological differences between the two hemiretinal systems may cause a false pathological diagnosis if retinal stimulus location is not taken into account.

Both latencies and scalp locations of the evoked components show differences between upper and lower hemiretinal stimulation. Component latencies reflect functional characteristics of the hemiretinal system, while the scalp location differences are caused by the different cortical regions which are activated according to the retinotopic representation of the visual field in the visual cortex. Upper retinal stimuli are routed to regions above the calcarine fissure, while lower retinal stimuli are transmitted to cortical areas below the calcarine fissure (see Sect. 6.1.2). Topographically, this results in a more posterior scalp location for components evoked by lower hemiretinal stimuli projected to the same horizontal eccentricity. In many subjects, these occipital components are located well below the inion, and due to this fact, in some subjects the components are missed (Lehmann and Skrandies 1979; Lesèvre and Joseph:1979). Scalp electrode arrays extending to below the inion are thus mandatory for the recording of electrical brain activity evoked by lower retinal stimuli.

\subsection{Complete Scalp Field Distributions: Pattern Reversal Evoked Activity Recorded in 45 Channels}

Among 25 subjects studied, Lehmann and Skrandies (1979) reported on multichannel recordings ( 45 channels) from five subjects using a checkerboard reversal stimulus. In this section, additional 45-channel data evoked by upper or lower hemiretinal checkerboard reversal stimulation are presented.

Simultaneous recordings in 45 channels were obtained in six adult healthy volunteers. The electrode array extended to $70 \%$ of the distance from the inion to the nasion and to $3 \mathrm{~cm}$ below the inion, and it covered laterally $70 \%$ of the distance between the two meati acustici (see inset, Fig. 10). Technical details of the multichannel recordings can be found in Lehmann and Skrandies (1979).

A checkerboard reversal stimulus (50' check size, $96 \%$ contrast) was presented on a circular field $\left(15.5^{\circ}\right.$ arc diameter) via a rotating mirror at 1.6 reversals per second monocularly to the upper or lower hemiretina. The data were topographically analyzed following the principles outlined in Sect. 2.

Figure 10 illustrates two map series of scalp potential fields evoked by upper or lower hemiretinal stimuli. Equipotential maps are constructed by entering the potential value of each electrode at its respective position, and points of equal potentials are connected by lines (as contour lines connect locations of equal altitude on geographical maps). Relative global field power curves are also plotted for both map series. Maximal field power occurs for upper retinal stimuli at $113 \mathrm{~ms}$ and for lower retinal stimuli at $121 \mathrm{~ms}$ (see arrowheads). The two map series show a grossly similar pattern of activity at different post-stimulus times. An occipital positivity surrounded by steep gradients develops around $100 \mathrm{~ms}$, 

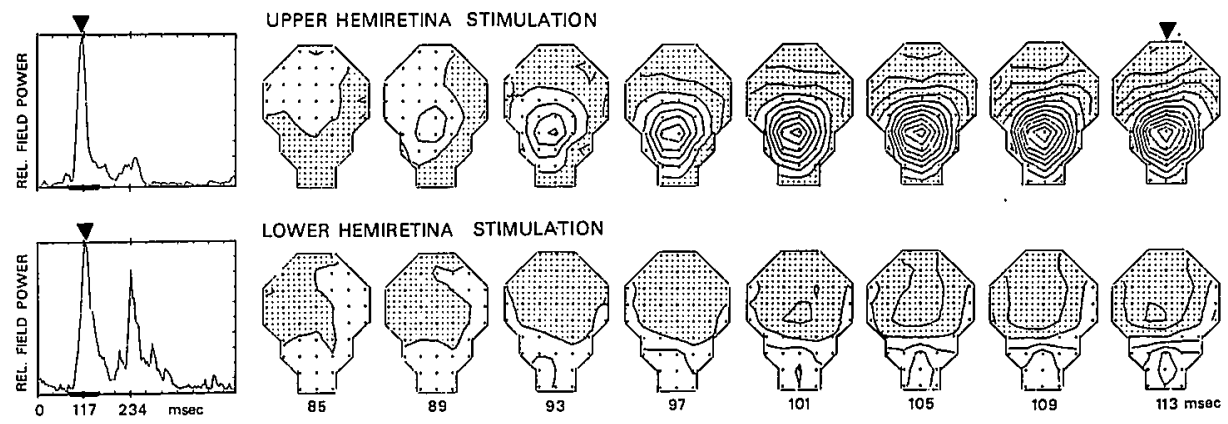

UPPER HEMIRETINA STIMULATION (CONT'D)
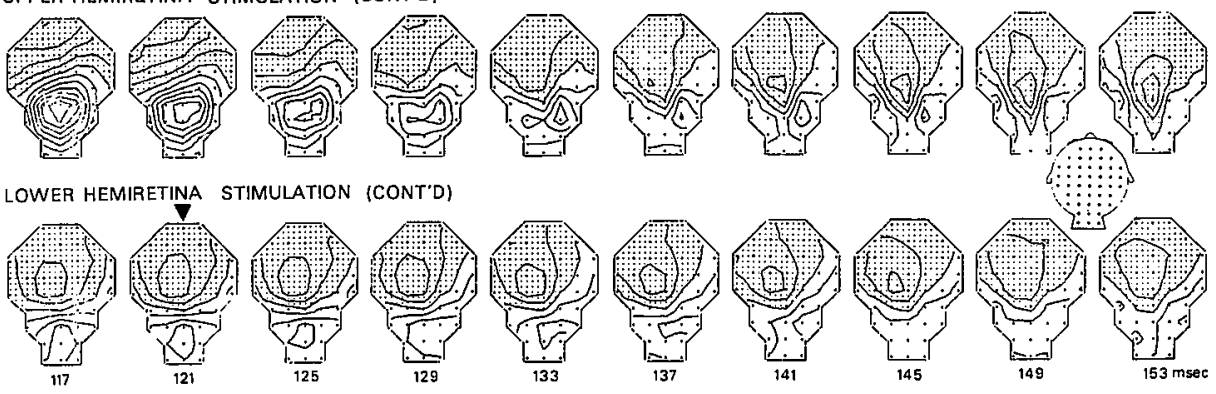

Fig. 10. Global field power curves (top left) and series of scalp field maps of potentials evoked by a checkerboard reversal stimulus ( $50^{\prime}$ check size, test field of $15.5^{\circ}$ arc diameter, $96 \%$ contrast) presented to the upper or lower hemiretina. The $68-\mathrm{ms}$ epoch during which maps are illustrated is indicated by horizontal bars in the field power plots. Simultaneous recordings in 45 channels as indicated in inset, most anterior electrode at $30 \%$ nasion-inion distance, circled electrode at inion. Dotted areas are negative, white areas positive with respect to the average reference. Equipotential lines in steps of $1 \mu \mathrm{V}$. Arrowheads in the field power graphs and over maps indicate times of maximal field power. Note the earlier latency time, and the more anterior scalp location of the occipital positive component for upper than lower hemiretinal stimuli. (From Lehmann and Skrandies 1984: by permission of Pergamon Press Oxford)

reaches its peak at component latency, and slowly disappears. This behavior is paralleled in time in the field power curves. There are also differences in the topographical distributions of the occipitally positive components, which show a more anterior scalp location of the components evoked by upper than by lower retinal stimuli.

The mean component latency of the six subjects was $99 \mathrm{~ms}$ for upper retinal stimuli and $115 \mathrm{~ms}$ for lower retinal stimuli. Longer component latencies for the lower retina were consistently found in all subjects, and the difference was statistically significant.

The scalp locations of the occipital positive components at individual times of maximal field power of the six subjects in the two stimulus conditions are illustrated in Fig. 11. In all subjects, components evoked by upper retinal stimuli are more anteriorly located than components evoked by lower retinal stimuli, resulting in a highly significant difference. 


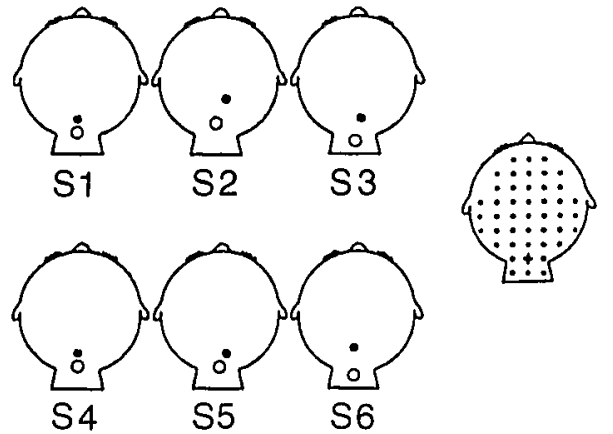

- UPPER - LOWER
Fig. 11. Scalp locations of potential field maxima at individual times of maximal field power around $100 \mathrm{~ms}$ latency of six subjects evoked by upper retinal (dots) and lower retinal (open circles) stimuli. Mean component latency for the upper retina was $99 \mathrm{~ms}$, for the lower retina $115 \mathrm{~ms}$. Recordings from an array of 45 electrodes as illustrated. (Data from Skrandies and Lehmann 1982)

\subsection{Generalization and Extension of Visual Evoked Potential Findings}

\subsubsection{Pattern Reversal Stimuli}

Differences between potential components evoked by checkerboard reversal stimuli presented to the upper or lower hemiretina were further investigated in ten other subjects using a midline row of electrodes over the posterior scalp areas. Such a reduced electrode array was chosen because the potential fields obtained with 45 channels show occipital components which are symmetric about the midline (see Fig. 10). Thus, a row of electrodes along the sagittal midline detects the major pertinent features of the scalp field distributions.

Nine recording electrodes were placed at regular intervals of $7 \%$ of the nasion inion distance along the midline between the vertex and the inion extending to $7 \%$ below the inion. Checkerboard reversal stimuli of $62 \%$ contrast (check size $30^{\prime}$ arc) were presented at a rate of 3.9 changes per second on a TV screen $\left(8.7^{\circ}\right.$ by $11.4^{\circ} \mathrm{arc}$ ) which was rotated by $90^{\circ}$ so that the display lines ran vertically and significant display time differences were avoided.

Occurrence times of maximal field power between 80 and $140 \mathrm{~ms}$ determined component latencies. Component locations at these times and the topographical behavior of potential maxima and minima over the whole recording epoch were analyzed.

Figure 12 shows the grand mean potential waveshapes computed from the data of all subjects evoked by upper or lower hemiretinal stimuli. The time of maximal field power occurs later for the lower than the upper hemiretina (see vertical lines). Analysis of the individual field power data of the subject population resulted in a mean component latency of $110.8 \mathrm{~ms}$ for upper and $118.1 \mathrm{~ms}$ for lower retinal stimuli, a significant difference $(t=4.45, p<0.0025)$.

Differences in topography may become more obvious in waveshapes when looking at the local curvature of the potential fields (see Sect. 2.4). Figure 13 illustrates how current source density data are obtained from the original data as the second derivative of the potential distribution with respect to space (Mitzdorf and Singer 1978; Mitzdorf 1985; McKay 1984a, b). The waveshapes display the change of the curvature of the field as a function of time, and thus the local characteristics of 
Fig. 12. Grand mean potential waveshapes of ten subjects evoked by upper (left) or lower (right) checkerboard reversal stimuli. Vertical lines, times of maximal field power. The electrode array starts at the vertex $(\mathrm{Cz})$ and extends along the sagittal midline to below the inion. The data are referred to the average reference

Fig. 13. Grand mean data (ten subjects) of potentials evoked by upper retinal stimuli. Potential waveshapes referred to the average reference (left) and corresponding current source density waveforms (right) illustrating the local curvature of the potential fields. Note that potential gradients become more pronounced in the current source density waveforms

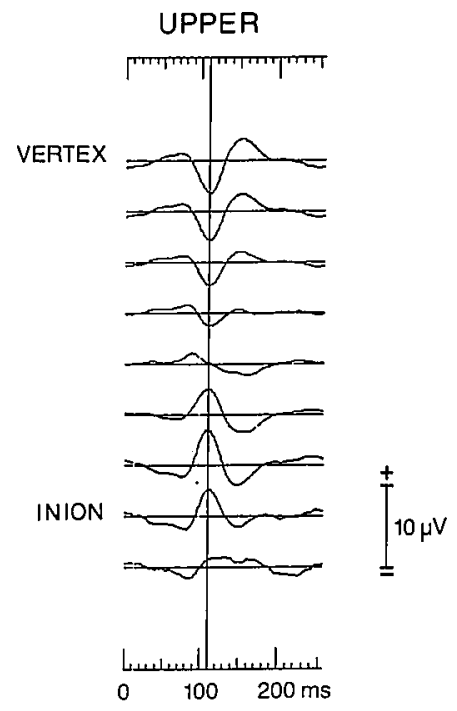

LOWER
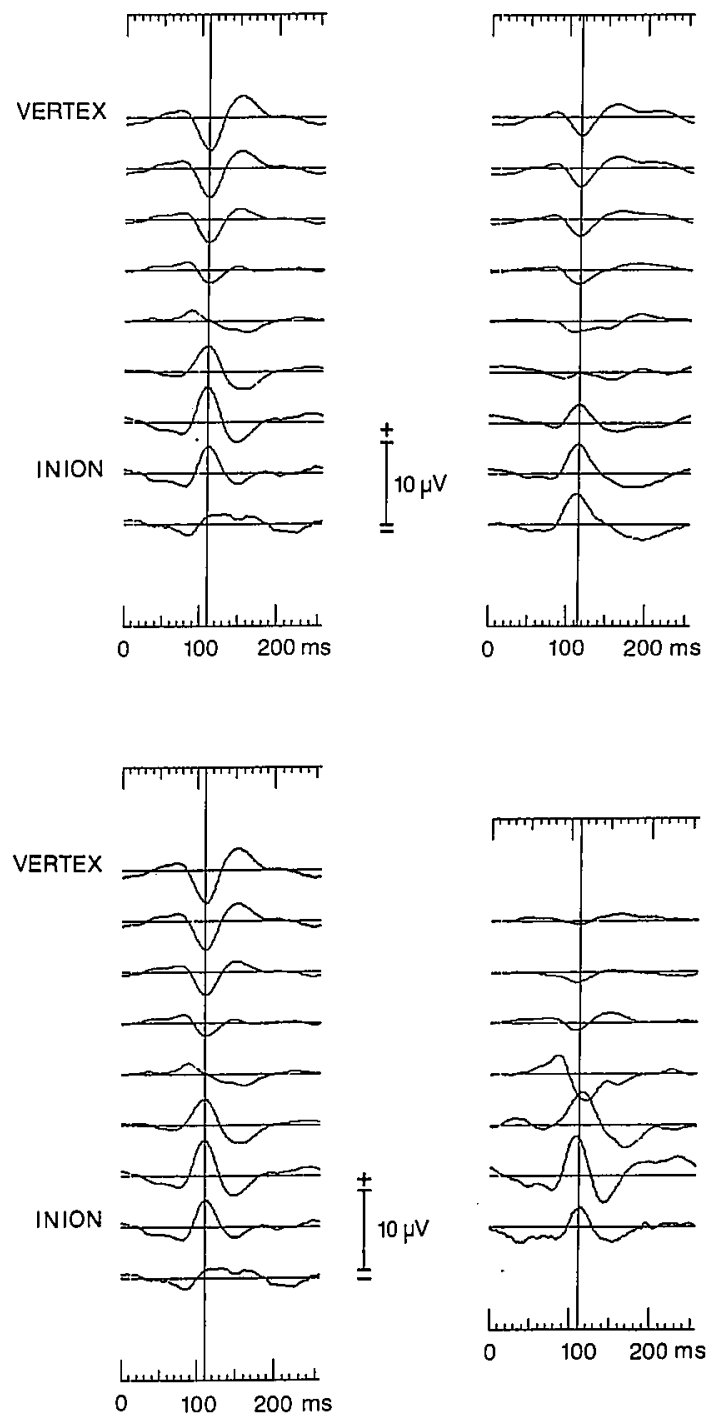

the potential fields are enhanced. The grand mean data evoked by upper and lower retinal stimuli are compared in the form of current source densities in Fig. 14: While for the lower hemiretina the occipital positivity around $100 \mathrm{~ms}$ is restricted to the last two electrode positions, the upper hemiretina evokes a positive component extending further anteriorly. At electrode 5 a positivity is seen following upper hemiretinal stimuli, and a negativity following lower hemiretinal stimuli. The potential gradient is by definition reference-independent, and it is important to note that the direct analysis of the scalp fields in terms of field power and component location yielded identical topographical conclusions from the dàta. 

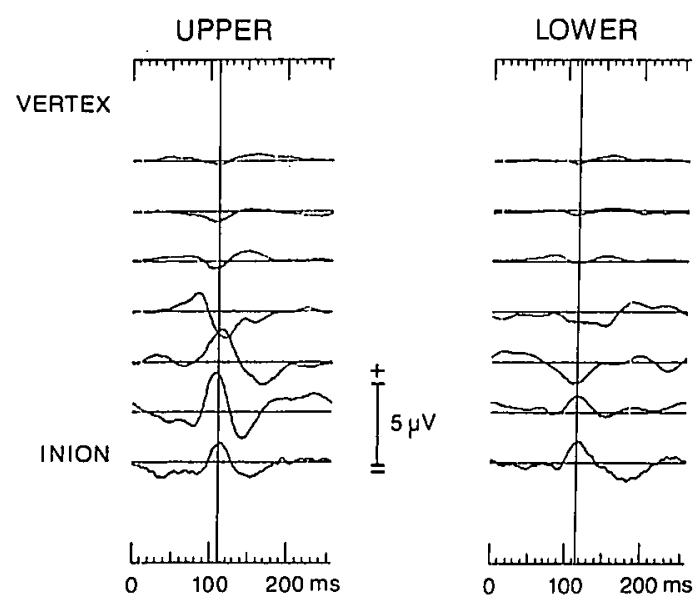

Fig. 14. Grand mean current source density waveforms of ten subjects evoked by upper or lower retinal stimuli illustrating the change of the curvature of the field as a function of time. This method enhances the local characteristics of the potential fields. The corresponding potential waveforms are shown in Fig. 13

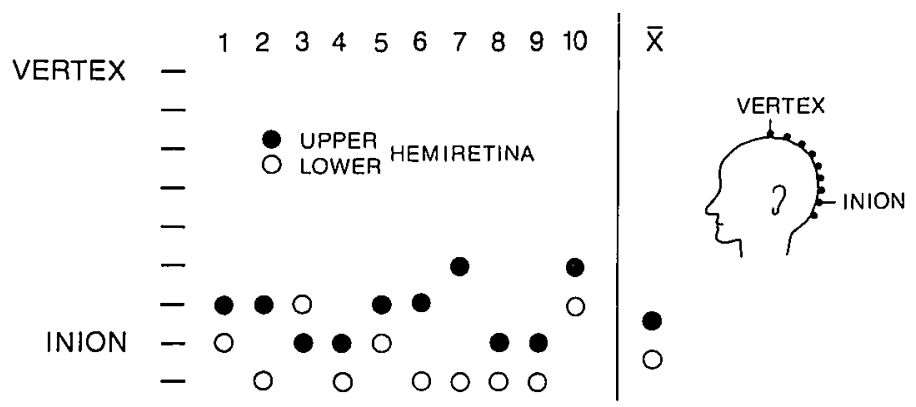

Fig. 15. Distribution of potential maxima evoked by upper or lower retinal checkerboard reversal stimuli along the sagittal midline. Electrode array as shown in head schema. Data of ten subjects obtained at times of individual maximal field power and population means are displayed. The location differences are highly significant (see text)

The distributions of the component locations (i.e., the potential maxima) of the ten subjects at their individual occurrence times of maximal global field power, as well as the mean component locations, are shown in Fig. 15. In nine of the ten subjects with upper hemiretinal stimuli, component locations are obtained which are anterior to those evoked by stimulation of the lower hemiretina, and the location differences were highly significant in the subject population $(t=3.88$, $p<0.0025$ ).

The differences of potential maxima and minima locations evoked by upper or lower retinal stimuli were further analyzed over the whole recording epoch of $256 \mathrm{~ms}$. It turned out that location differences were not restricted to component latencies, but that the differences appear to be related to the complete neural activation cycle.

Figure 16A illustrates the mean potential maxima locations and indicates significance levels of the location differences between upper and lower retinal stimuli computed on the individual location data of the ten subjects. The mean locations of the potential minima and the corresponding significance levels are shown in Fig. 16B. 

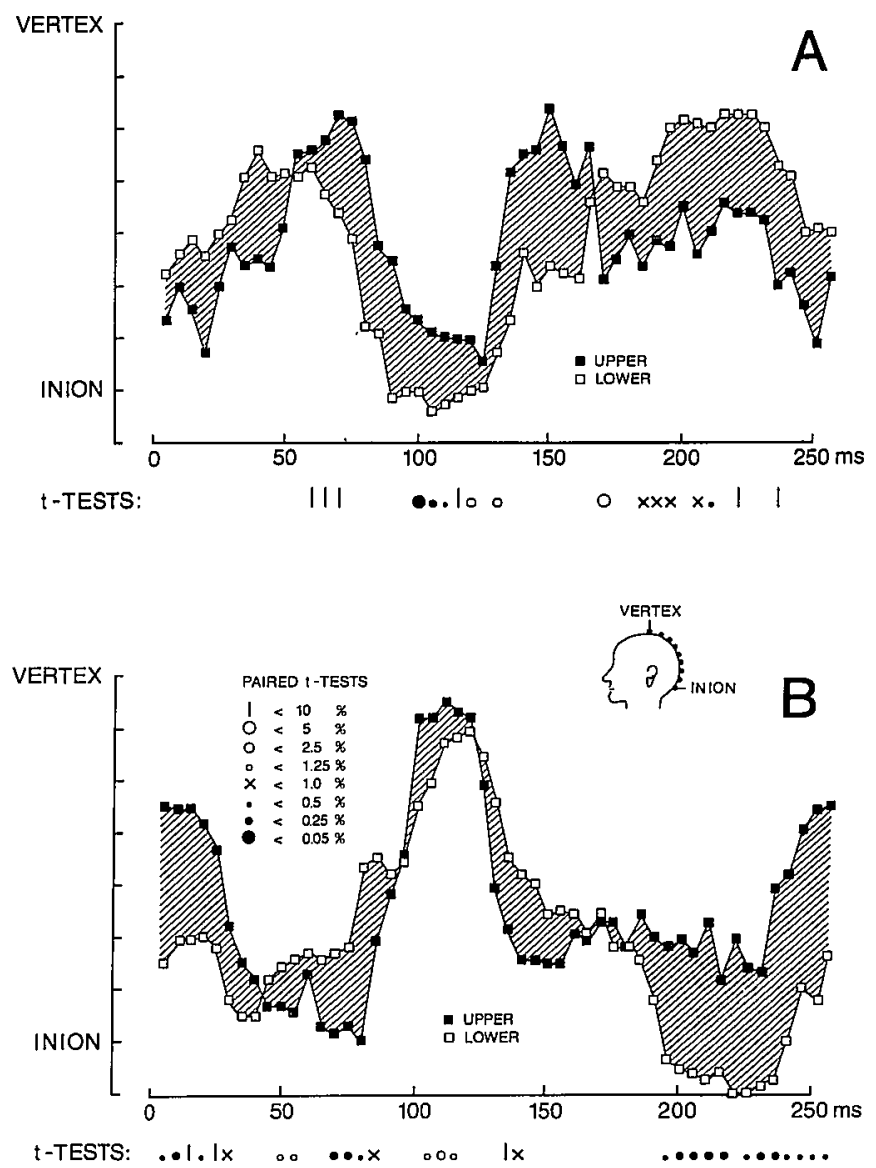

Fig. 16A, B. Mean potential maxima (A) and minima locations (B) of ten subjects evoked by upper or by lower retinal stimuli plotted between 5 and $255 \mathrm{~ms}$ at intervals of $5 \mathrm{~ms}$. Hatched areas indicate differences in location. The results of paired t-tests which were computed on the individual data are shown below each graph. Significant differences persist. over longer time epochs. Note clusters of high significance levels

The general distribution pattern for the maxima and minima is complementary in the anterior - posterior direction: during the analysis epoch the maxima exhibit up to about $80 \mathrm{~ms}$ an anterior, around $100 \mathrm{~ms}$ a posterior, and after about $130 \mathrm{~ms}$ again a more anterior scalp location, while the locations of the minima display a posterior - anterior - posterior sequence. This topographical pattern is very similar for brain activity evoked by upper and by lower retinal stimuli.

Most locations of the potential maxima evoked by upper or lower retinal stimuli are significantly different around $100 \mathrm{~ms}$, and also around $200 \mathrm{~ms}$ (Fig. 16A). Significant differences of the minima locations are found at early times (up to about $30 \mathrm{~ms}$ ) and around $100 \mathrm{~ms}$, and high significance levels also cluster between about 190 and $255 \mathrm{~ms}$ (Fig. 16B).

The very consistent results suggest that different neural generating mechanisms are activated by upper and lower retinal stimuli: The brain electrical activation 
patterns corresponding to upper retinal stimulation are located more anteriorly on the scalp. Significant location differences are not only observed at component latencies, but they prevail over long durations. Differences are still found at long latency times after $200 \mathrm{~ms}$ (see Fig. 16). The long-lasting influence of physical stimulus parameters on visual evoked brain activity is in agreement with earlier findings. Late components of the VEP related to the spatial frequency of the stimulus with latencies of $188 \mathrm{~ms}$ and $335 \mathrm{~ms}$ have been reported for lateralized checkerboard stimuli (Skrandies 1981). In addition, the scalp topography of late endogenous components such as the P300 component, which is related to cognitive information processing, as first described by Sutton et al. (1965), was also found to be affected by retinal stimulus location (Skrandies 1983).

\subsubsection{Different Presentation Modes: Onset, Offset, and Reversal}

Patterned visual stimuli have been used successfully for the study of evoked potentials in man. VEPs elicited by contrast stimuli presented without gross luminance changes are more reliable and show less intersubject variation than flash VEPs, and have thus become standard for clinical purposes (e.g., Halliday et al. 1973). Differences in waveshape morphology evoked by pattern appearance (onset), disappearance (offset), or contrast reversal of an identical checkerboard pattern have been described by various authors (Estevez and Spekreijse 1974; Kriss and Halliday 1980). In a pilot study, Skrandies et al. (1980) demonstrated significant differences between potential components evoked by different presentation modes of an identical checkerboard pattern, and between upper and lower hemiretinal stimuli. These differences were found both in component latency and scalp location.

Scalp potential distributions of VEPs evoked by pattern onset, offset, and reversal stimuli presented to the upper or lower hemiretina were further investigated in 13 subjects using a midline row of nine recording electrodes.

In this section, it will be shown that different presentation modes of an identical checkerboard pattern evoke occipitally positive components which occur at different latencies (around $100 \mathrm{~ms}$ ), and show scalp distribution differences suggesting the activation of non-identical neuronal populations. Simple waveshapes similarity between the reversal response and the offset response (Estevez and Spekreijse 1974; Kriss and Halliday 1980) does not appear to be sufficient evidence that these electrophysiological measures reflect identical underlying neuronal generating mechanisms, since different presentation modes give rise to neural mass responses which show distinct differences in time and scalp distribution, as will be shown below. In addition, these data demonstrate that the upper and lower retina behave as independent systems, displaying differences in component latency and in scalp location also with other stimulus presentation modes (Skrandies 1984a).

Thirteen healthy volunteers between 19 and 29 years of age (mean $=$ median $=22$ years) with normal or corrected-to-normal vision participated in the experiments. Nine Grass gold-cup electrodes were placed at regular intervals of $7 \%$ of the nasion - inion distance along the midline, starting at the vertex $(\mathrm{Cz})$ and extending to $7 \%$ below the inion. The most anterior electrode served as recording reference. 
The EEG was amplified (bandpassed from 0.1 to $70 \mathrm{~Hz}$ ), A/D-converted at a rate of 1000 samples per second and channel over a period of $512 \mathrm{~ms}$, averaged $(n=120)$, and stored on disc by a PDP11-40 computer.

Checkerboard reversal stimuli of $62 \%$ contrast were presented at a rate of 3.9 changes per second on a TV monitor $\left(8.7^{\circ}\right.$ by $11.4^{\circ}$ arc). Check size was either $60^{\prime}$ arc or $30^{\prime}$ arc. In the onset and offset conditions, the checkerboard was replaced every $256 \mathrm{~ms}$ by a homogeneous field of the same average luminance of 13.6 candela $/ \mathrm{m}^{2}$. When comparing upper and lower retina stimulation, significant display time differences inherent to a TV system were avoided by rotating the TV screen by $90^{\circ}$ so that the display lines ran vertically. For hemiretinal stimulation, the subject fixated small marks at the upper or lower border of the screen from a distance of $130 \mathrm{~cm}$.

The 12 experimental conditions (onset/offset/reverse * upper/lower hemiretina * large/small checks) were presented in a quasi-randomized order. Onset and offset responses were obtained in the same run. Each condition was presented three times; reliability of the data was confirmed by computing correlation coefficients between the first and the last run of the same experimental condition of each recording session using evoked potential waveshapes recorded at each electrode separately. Corresponding data of one subject participating in the experiment twice at a 1-week interval were also cross-correlated in order to determine the stability of the results.

The occurrence time of the maximal potential range between 80 and $160 \mathrm{~ms}$ coincided in most cases with the maximum of the first spatial derivative (i.e., the potential gradient) and defined component latency. In some subjects the onset responses were dominated by long latency components (as described by Kriss and Halliday 1980) or the lower hemiretinal responses displayed low signal-to-noise ratios, and in these cases (about 15\%) the secondary maximal values of the potential range function were included as an additional criterion. Comparison of this strategy with the unrestricted component definition did not reveal any differences regarding the conclusions derived from the data.

For statistical analyses, mean values of component latency and location were derived from repeated measurements in each subject and were entered in separate three-factorial analyses of variance. Significant influences were further examined by computing t-tests using adjusted alpha levels according to Holm's (1979) procedure. All results presented were significant at an alpha level of $p<0.005$.

\subsubsection{Reliability of the Data}

The mean correlation coefficients over all experimental conditions estimating the within-session reliability ranged between 0.60 and 0.90 , with a mean value over all subjects of 0.75 . The replication of the experiment in one subject at an interval of 1 week yielded a mean correlation of $r=0.83$ (range 0.74-0.93). Since all 256 time points were used for computing the correlations, not only major components but also very early and late parts of the VEP contribute in the same way to the general waveshape similarity indexed by the correlation coefficients. Thus, the values reported above indicate both a high overall similarity of the repeated recordings and reliability of the data. 
ON

A

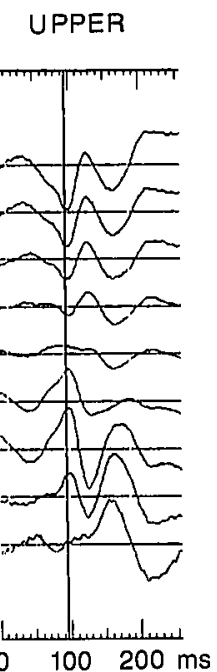

LOWER

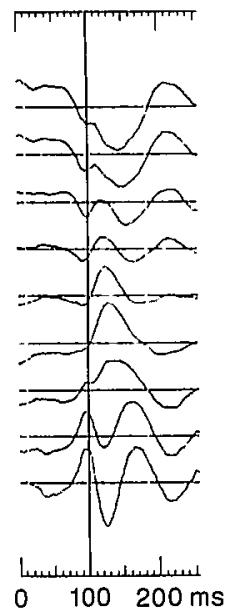

UPPER

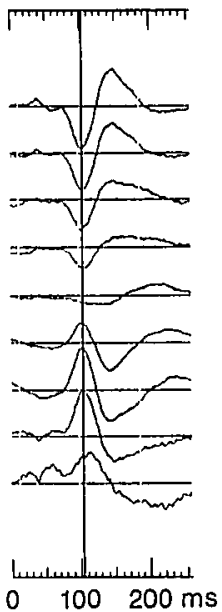

\section{REVERSE}

\section{LOWER}

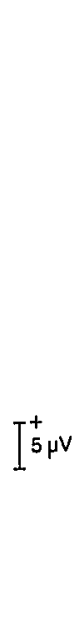

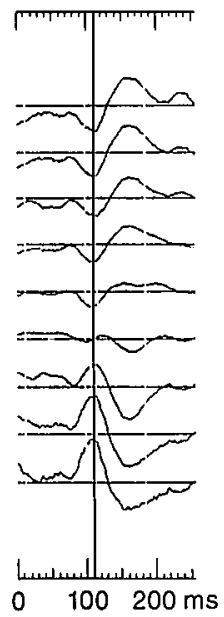

\section{OFF}

C

UPPER

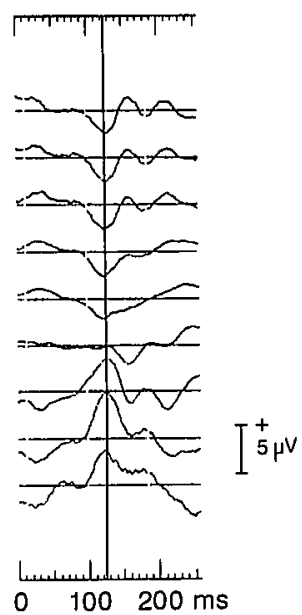

LOWER

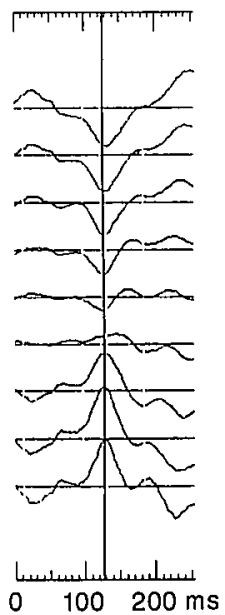

Fig. 17A-C. Original potential waveshapes evoked by small checks presented to the upper or lower retina. Recordings are obtained from a row of nine midline electrodes between the vertex (first trace) and 7\% below the inion. A checkerboard pattern (check size $30^{\prime}$ arc) was presented as onset (A), reversal (B), or offset stimulus (C). Average reference data from one subject

\subsubsection{Differences of Component Latencies}

Figure 17 illustrates sets of original VEP waveshapes of one subject evoked by a checkerboard presented to the upper or lower hemiretina as onset (Fig. 17A), reversal (Fig. 17B) or offset stimulus (Fig. 17C). The major component in all experimental conditions is an occipital positivity occurring around $100 \mathrm{~ms}$ latency, and there appear systematic differences in component latency, as illustrated by the 
Table 1. Mean component latencies (ms) of 13 subjects and latency differences between stimulus conditions

\begin{tabular}{|c|c|c|c|c|c|}
\hline Stimulus & On & Difference & Reverse & Difference & Off \\
\hline Upper hemiretina & 94.2 & $13.9 * * *$ & 108.1 & $15.9 * * * *$ & 124.0 \\
\hline Difference & $16.5 * * *$ & & $8.6^{* * *}$ & & $10.4 * * *$ \\
\hline Lower hemiretina & 110.7 & $6.0 * *$ & 116.7 & $17.7 * * * *$ & 134.4 \\
\hline
\end{tabular}

*** $p<1 \%$;** $p<5 \%$ : t-tests corrected according to Holm's procedure

potential range plots in the corresponding Figs. 18A-C. The vertical lines in Fig. 17 indicate times of maximal field power, and one sees that the component latencies are different for different presentation modes as well as for upper and lower retinal stimuli.

The occipital positive component evoked by pattern onset showed consistently shorter latencies (mean over all subjects: $102.5 \mathrm{~ms}$ ) than that evoked by reversal (mean: $112.4 \mathrm{~ms}$ ), or offset stimuli (mean: $129.2 \mathrm{~ms}$ ). Three-factorial analyses of variance showed significant effects of presentation mode and of retinal location on component latencies. Check size was not a significant factor and also did not show up in interactions. Thus, the mean component latencies evoked by large and small stimuli were used for further analysis. Table 1 summarizes the latency data and indicates significant differences revealed by paired t-tests with alpha levels corrected for multiple testing according to Holm's (1979) procedure. Onset stimuli yielded significantly shorter latencies than reversal or offset stimuli for both the upper and lower hemiretina, while offset stimuli consistently showed the longest latencies.

Significant latency differences were also found when comparing component latencies evoked by upper or lower retinal stimulation: Upper hemiretinal stimuli yielded shorter latencies than lower hemiretinal stimuli. This finding was significant for all three presentation modes (see Table 1).

The finding that onset stimuli yielded significantly shorter latencies than offset stimuli is supported by other data on motor reaction time. Pease and Sticht (1965) reported that the reaction times to stimulus onset were significantly shorter than those to stimulus offset when the light stimuli were presented to the peripheral retina. In the fovea no statistically significant reaction time differences were observed. This observation was confirmed by Bartlett et al. (1968), who found significantly shorter reaction times to onset than to offset stimuli. These differences were independent of the wavelength of the stimulus.

\subsubsection{Differences of Component Locations}

Topographical differences between conditions were revealed by comparison of the data as potential profiles on the scalp plotted over time (see Sect. 2.1.2). Figure 18 illustrates the data of Fig. 17 in the form of series of topographical potential profiles. In each profile series one sees that during the first $60 \mathrm{~ms}$ a flat potential distribution prevails, and then an occipital positivity develops which reaches its 

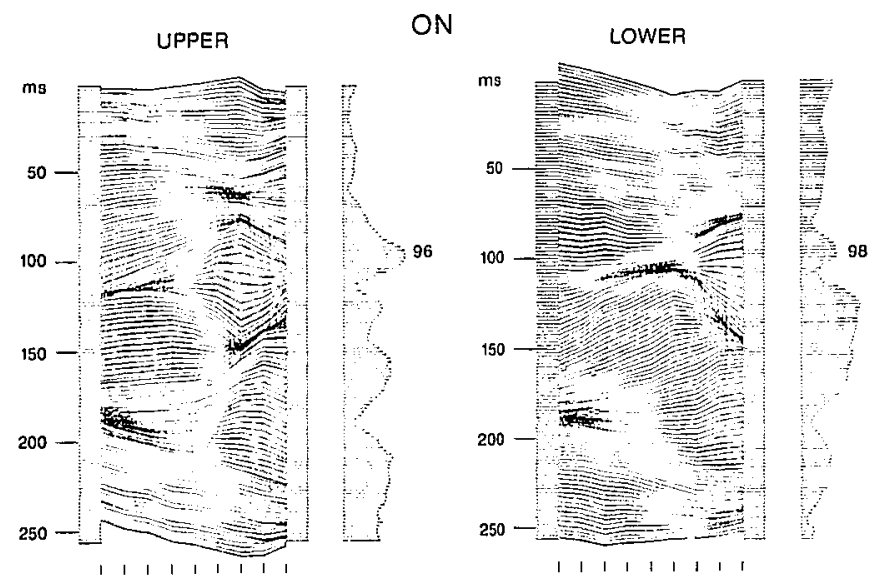

A ANTERIor

POSTEAIOR

$$
\text { ANTERIOR POSTERIOR }
$$

REVERSE
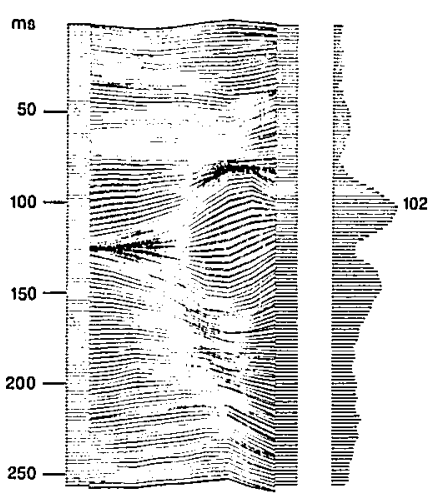

B ANTERIOR POSTERIOR
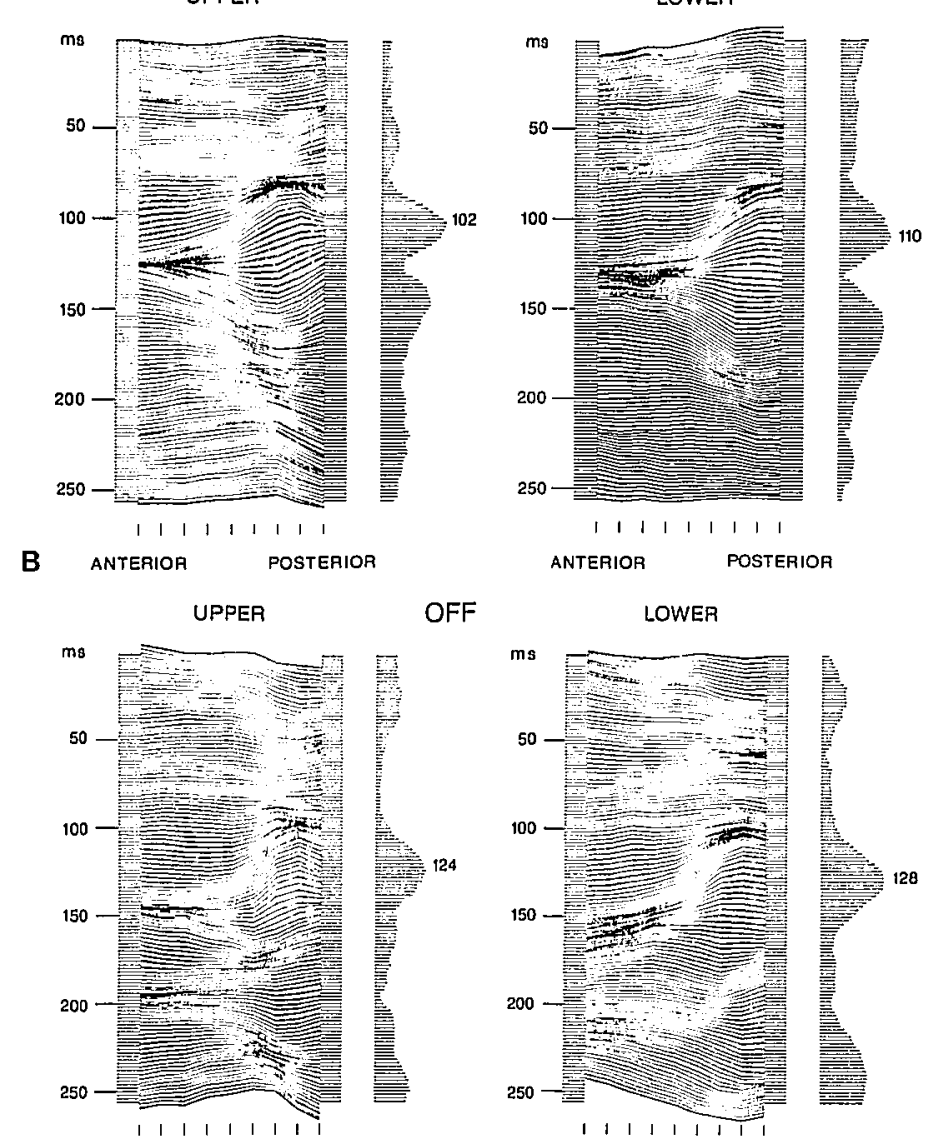

OFF

ANTERIOR POSTERIOR

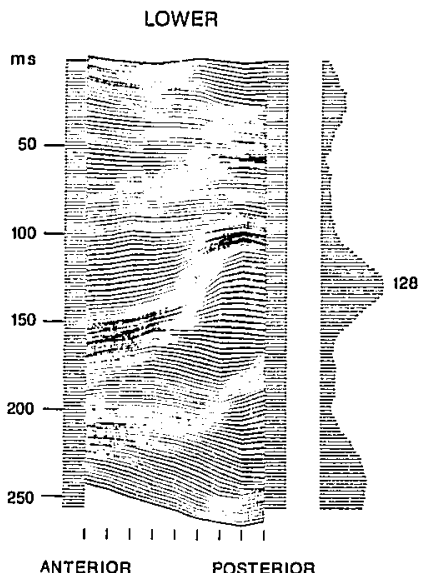

Fig. 18A-C. Data of Fig. 17 displayed topographically as series of potential profiles. Adjacent to each profile series, global field power is plotted. Different presentation modes yield topographical differences. A Onset; $\mathbf{B}$ reversal; $\mathbf{C}$ offset stimuli 
maximum between 96 and $128 \mathrm{~ms}$ (depending on the stimulus condition). Later this maximum is replaced by a potential minimum. This behavior over time is similar to that of the complete scalp field map series illustrated in Fig. 10. The amount of activity quantified by the global field power at each instant is plotted as a function of time adjacent to each profile series in Fig. 18.

Although the potential profiles in Fig. $18 \mathrm{~A}-\mathrm{C}$ are globally similar, there are distinct differences: Not only the latencies but also the respective component locations appear to depend on presentation mode and retinal stimulus position. Three-factorial analyses of variance yielded significant effects of presentation mode and retinal location as well as a significant interaction of these factors. This indicates that different presentation modes do not display the same location differences between components evoked by stimulation of the upper or lower hemiretina. As with the latency data, check size turned out not to be a significant factor, and mean values of the location data evoked by large and small checks were used for comparisons with paired t-tests. Again, alpha levels were corrected for multiple comparisons (Holm 1979).

The results on component location are summarized in Table 2. For the upper hemiretina all component locations were significantly different from each other depending on presentation mode: pattern onset components were located most anteriorly (mean $5.14 \mathrm{~cm}$ above the inion) and offset components (mean location at the inion). On the other hand, components evoked by lower hemiretinal stimulation displayed no significant location differences according to presentation mode.

Highly significant differences were observed for onset and reversal stimuli when comparing retinal location: The components were located more posteriorly following lower hemiretinal stimulation. Components evoked by offset stimuli showed no significant differences between upper and lower hemiretinal stimuli. This may be explained by the fact that the components evoked by upper offset stimuli are already located far posteriorly on the scalp, and with lower retinal stimuli no recordable differences are present.

Figure 19 shows the grand mean potential profiles of the 13 subjects in all stimulus conditions which were investigated. The profiles were constructed at the individual occurrence times of the components and then averaged across subjects. The topographical differences discussed above are also evident in the grand mean data.

Table 2. Mean component locations of 13 subjects and location differences between stimulus conditions

Mean peak position ( $\mathrm{cm}$ above inion)

\begin{tabular}{llllll}
\hline Stimulus & On & Difference & Reverse & Difference & Off \\
\hline Upper hemiretina & 5.14 & $3.09^{* * *}$ & 2.05 & $2.05^{* * * *}$ & 0.00 \\
Difference & $4.63^{* * *}$ & & $2.56^{* * * *}$ & & 0.77 \\
Lower hemiretina & 0.51 & 1.02 & -0.51 & 1.28 & 0.77 \\
\hline
\end{tabular}

*** $p<1 \%$ : t-tests corrected according to Holm's procedure 

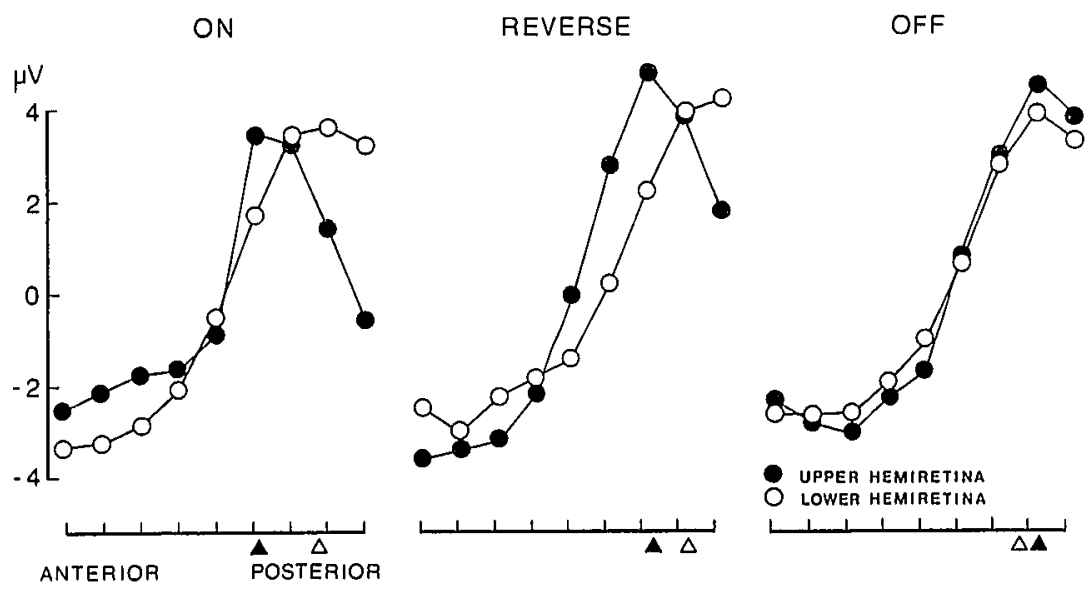

Fig. 19. Grand mean potential profiles of 13 subjects in the various stimulus conditions at individual times of maximal field power. Note location differences between upper and lower retinal stimuli as well as differences between presentation modes. Arrowheads indicate mean component locations

Additional paired t-tests confirmed that there were no significant differences between conditions in terms of potential range, amplitude, or gradient. The global appearance of the scalp distributions is rather similar, showing an occipital positive component around $100 \mathrm{~ms}$ latency. Small but statistically significant changes occur mainly in the location of the maximal scalp field values (arrowheads in Fig. 19, see also Table 2). This suggests that similar neural generating mechanisms taking place at different post-stimulus times are activated within different regions of the occipital cortex.

\subsubsection{Analysis of Waveshape Similarity}

As the detailed topographical analysis shows, visual potentials evoked by different presentation modes of an identical checkerboard pattern yield different neural activation patterns. These differences are both in the time domain (i.e., component latencies) and in the spatial distribution of the evoked electrical fields. None of the presentation modes showed sufficient similarity to support the identity of the potentials evoked by offset and reversal that has been suggested (Estevez and Spekreijse 1974; Kriss and Halliday 1980). Also, a simple summation of onset and offset responses does not yield the same pattern of activity as that evoked by reversal stimuli. Figure 20 illustrates this point: In all recording channels the computed sum of onset and offset response waveforms looks different from the potentials evoked by a checkerboard reversal stimulus. The question of waveshape similarity was approached quantitatively by computing cross-correlation functions between corresponding channels evoked in the different stimulus conditions. Table 3 shows the numerical results, giving the mean and the median correlation coefficients as well as the maximal correlation obtained in any one of the nine recording channels. The data in the upper right triangle of the matrix of Table 3 

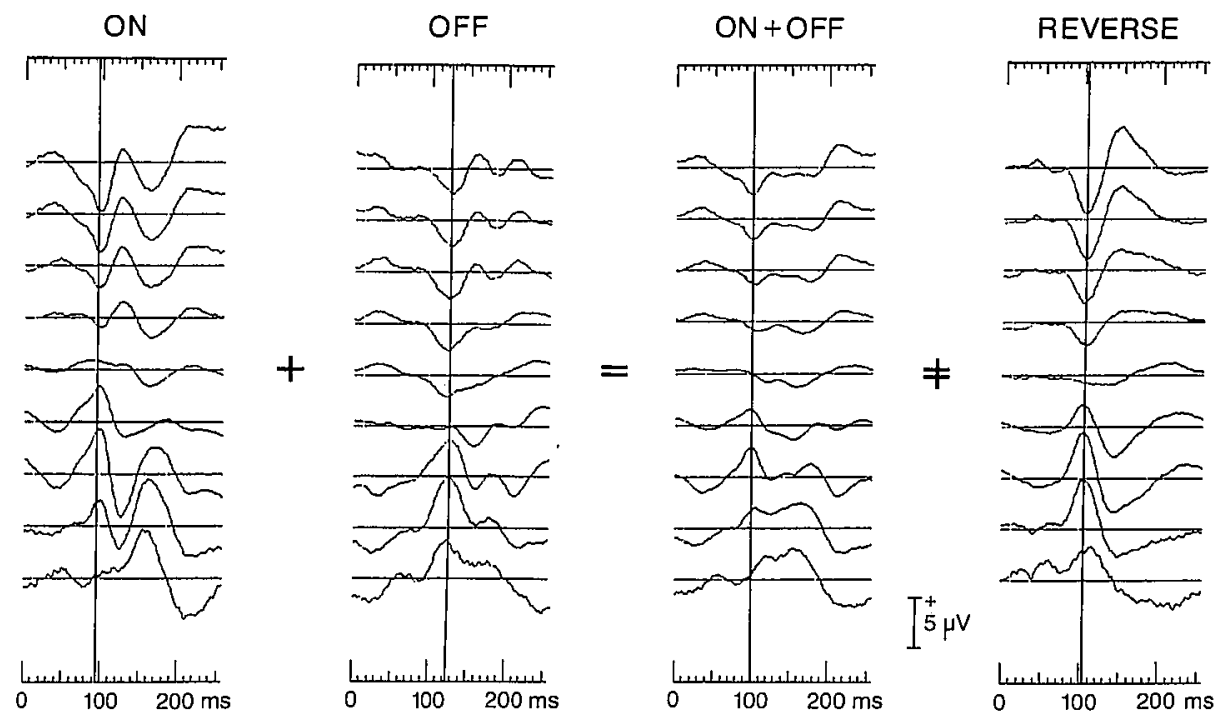

Fig. 20. Potential waveshapes evoked by stimuli presented to the upper retina as onset, offset, or reversal presentations. The computed mean of onset and offset evoked potentials $(O N+O F F)$ is different from the reversal potential waveshapes

Table 3. Correlation coefficients computed between potential waveshapes from different stimulus conditions for corresponding recording channels

\begin{tabular}{l|l|l|l|l|l}
\hline Maximum & & On & Off & Reversal & On +off \\
\hline On & Mean & & $\begin{array}{r}0.05 \\
-0.01 \\
0.58\end{array}$ & $\begin{array}{l}0.06 \\
0.07 \\
0.30\end{array}$ & $\begin{array}{l}0.77 \\
0.79 \\
0.91\end{array}$ \\
\hline Off & Maximum & & & 0.29 & 0.64 \\
& Mean & 0.52 & & 0.22 & 0.60 \\
& Median & 0.55 & & 0.63 & 0.87 \\
\hline \multirow{2}{*}{ Reversal } & Maximum & 0.65 & & & 0.25 \\
& Mean & 0.66 & 0.66 & & 0.17 \\
& Median & 0.68 & 0.66 & & 0.76 \\
\hline \multirow{2}{*}{ On+off } & Maximum & 0.84 & 0.76 & & \\
& Mean & 0.78 & 0.71 & 0.72 & 0.75 \\
& Median & 0.79 & 0.79 & 0.79 & \\
\hline
\end{tabular}

Mean and median values are derived from the correlations in all nine channels, maxima give the largest correlation coefficients obtained independent of the channel. Upper right triangle lists correlation coefficients at time zero (i.e., no time shift allowed). Lower left triangle gives correlation coefficients derived from maxima of whole cross correlation functions 


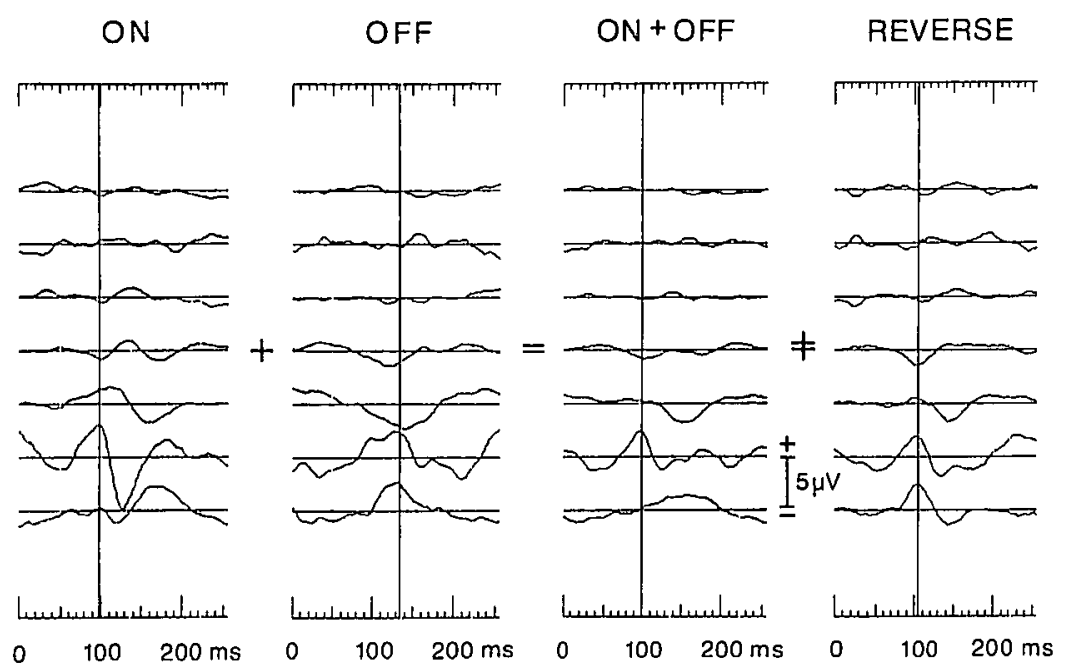

Fig. 21. Current source density waveforms evoked by onset, offset, and contrast reversal stimuli illustrating the change of the curvature of the potential field as a function of time. The computed sum of onset and offset evoked brain activity is also included. Note pronounced differences in latency and in local potential field curvature between stimulus conditions. The corresponding potential waveshapes are shown in Fig. 20

correspond to the correlations obtained at time zero (i.e., when not considering any time shifts present), and one sees that both onset and offset evoked potentials are more similar to the computed sum (the mean of onset and offset responses) than the reversal condition, which yields a mean correlation of only $r=0.25$. When the maxima of the cross-correlation functions are analyzed (i.e., when the time series are shifted against each other, and only the maximal correlation coefficient is considered), basically the same result is obtained. These correlation coefficients, given in the lower left triangle of the matrix of Table 3, are higher than the other values. This is to be expected, since the curves have been shifted with respect to each other in order to maximize similarity. But still the correlations between the computed sum of onset and offset responses and the reversal responses are smaller than the correlation between onset or offset responses and the computed mean waveshapes. As a result of the correlation analysis it is clear that the potential waveshapes also show differences, and that there is no compelling similarity between any of the potentials evoked by the different presentation modes.

In addition, the current source density of these data was computed, resulting in the waveshapes shown in Fig. 21. This figure illustrates the second spatial derivative, which by definition is independent of the reference electrode, and it is obvious that stimulus onset, offset, and reversal evoked spatially different electrical brain activity patterns. The latency differences described above are confirmed (see vertical lines in Fig. 21), and the largest changes in the curvature of the evoked scalp fields occur in the onset condition further anterior on the scalp than with checkerboard offset or reversal. Comparing the activity of channel 5, it becomes obvious that reversal and offset are also different. These data further support the conclusion that electrical brain activity elicited by contrast reversal 
is not identical to a simple summation of the brain activity evoked by stimulus appearance and disappearance.

\subsubsection{Grating Stimuli}

In another population of 11 adult subjects, recordings in 16 channels to a reversing vertical grating stimulus presented to the upper or lower hemiretina were obtained. Scalp field distributions evoked by stimuli of different spatial frequencies show differences in component latency and potential range, and such multichannel data are discussed in detail elsewhere (Skrandies 1984b). In the present experiments it was found that also with grating stimuli, the evoked scalp field distributions of all subjects display differences both in component latencies and in topography, depending on retinal stimulus location.

Evoked potentials were recorded in 11 healthy subjects (between 19 and 42 years of age) from an electrode array consisting of 16 electrodes and extending in four regular steps from $2 \mathrm{~cm}$ below the inion to $7 \mathrm{~cm}$ above the inion, and in two steps to $6 \mathrm{~cm}$ left and right from the midline (see inset in Fig. 22). A frontal midline electrode served as reference for recording. For amplification, Grass P511 amplifiers with a bandpass between 0.1 and $100 \mathrm{~Hz}$ were used, and the data were A/D-converted at a rate of 1000 samples per second and channel over a period of $256 \mathrm{~ms}$, averaged $(n=90)$, and stored on disc for later analysis.

Square-wave-modulated grating stimuli of $80 \%$ contrast were presented at a rate of two reversals per second by a TV stimulation system as a circular test field of $7^{\circ}$ arc diameter. Vertical gratings of 2.3 cycles per degree were presented to the upper or lower retina from a distance of $185 \mathrm{~cm}$.

Potential field maps were constructed from the data, and global field power defined the component latencies. Differences of component latencies and locations were tested using paired t-tests.

Figure 22 shows the grand mean potential waveshapes evoked by upper hemiretinal stimuli. Vertical lines indicate times of maximal field power (see also Fig. 26). In the lower part the construction of the potential field map at component latency is illustrated. A series of potential distribution maps is illustrated in Fig. 23 in which the data of Fig. 22 are shown between 80 and $138 \mathrm{~ms}$ with intervals of $2 \mathrm{~ms}$.

Grand mean potential waveshapes computed from the data of all subjects evoked by lower hemiretinal stimuli are illustrated in Fig. 24. The scalp distribution at component latency shows a potential maximum shifted posteriorly as compared to the activity evoked by upper retinal stimuli. The whole series of scalp field maps is different from that evoked by upper hemiretinal stimuli in terms of component location (see Fig. 25). Latency differences determined by global field power are shown in Fig. 26: The upper hemiretina reaches its maximum at $97 \mathrm{~ms}$, the lower hemiretina at $99 \mathrm{~ms}$. These figures refer to the grand mean data (i.e., the mean evoked activity of the 11 subjects studied), and paired t-tests on the individual data revealed consistent, statistically significant differences. The mean latency for the upper hemiretina was $96.4 \mathrm{~ms}$, while that for the lower hemiretina was $100.7 \mathrm{~ms}$ in the subject population. This difference was significant $(t=4.66$, $p<0.0005$ ). 

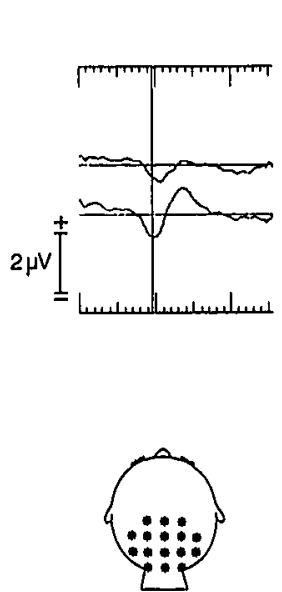

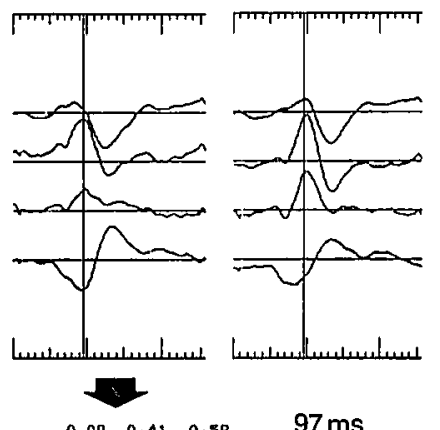

$\begin{array}{lllll}-0.10 \quad 1.47 & 1.55 \quad 1.16 & -0.17\end{array}$

$\begin{array}{lllll}-0.76 & 0.72 & 1.31 & 0.05 & -0.52\end{array}$

$-0.98-0.57-0.93$
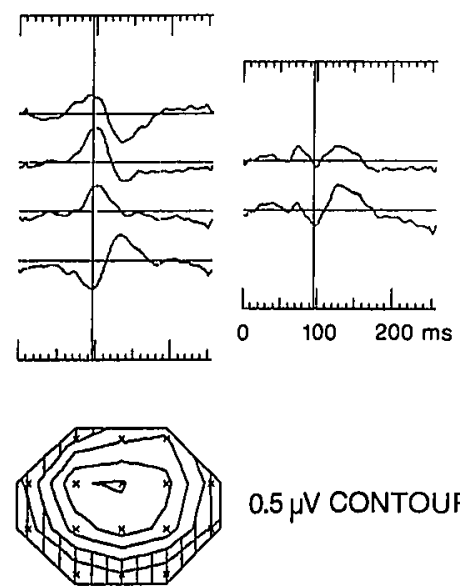

$0.5 \mu \mathrm{V}$ CONTOURS

Fig. 22. Grand mean evoked potential waveshapes of 11 subjects evoked by upper hemiretinal stimulation, recorded from an array of 16 electrodes extending in four regular steps from $2 \mathrm{~cm}$ below the inion to $7 \mathrm{~cm}$ above the inion, and in two steps to $6 \mathrm{~cm}$ to the left and right of the midline (see inset). At component latency (maximal field power at $99 \mathrm{~ms}$ ) the construction of a potential map is illustrated: the microvolt values of each electrode are entered at their respective positions (lower left), and areas of equal potential values are connected by lines. This results in an equipotential line map (lower right). (From Skrandies 1986a: by permission of Butterworth Publishers, Stoneham)

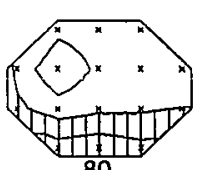

80
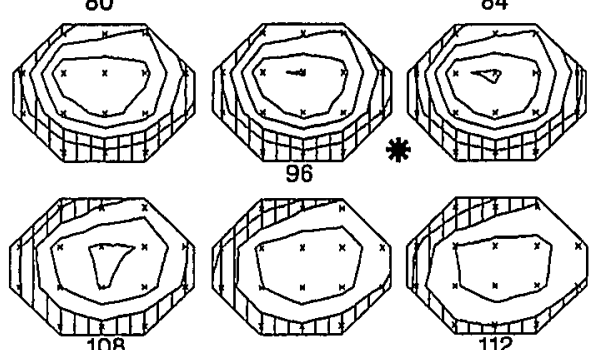

112
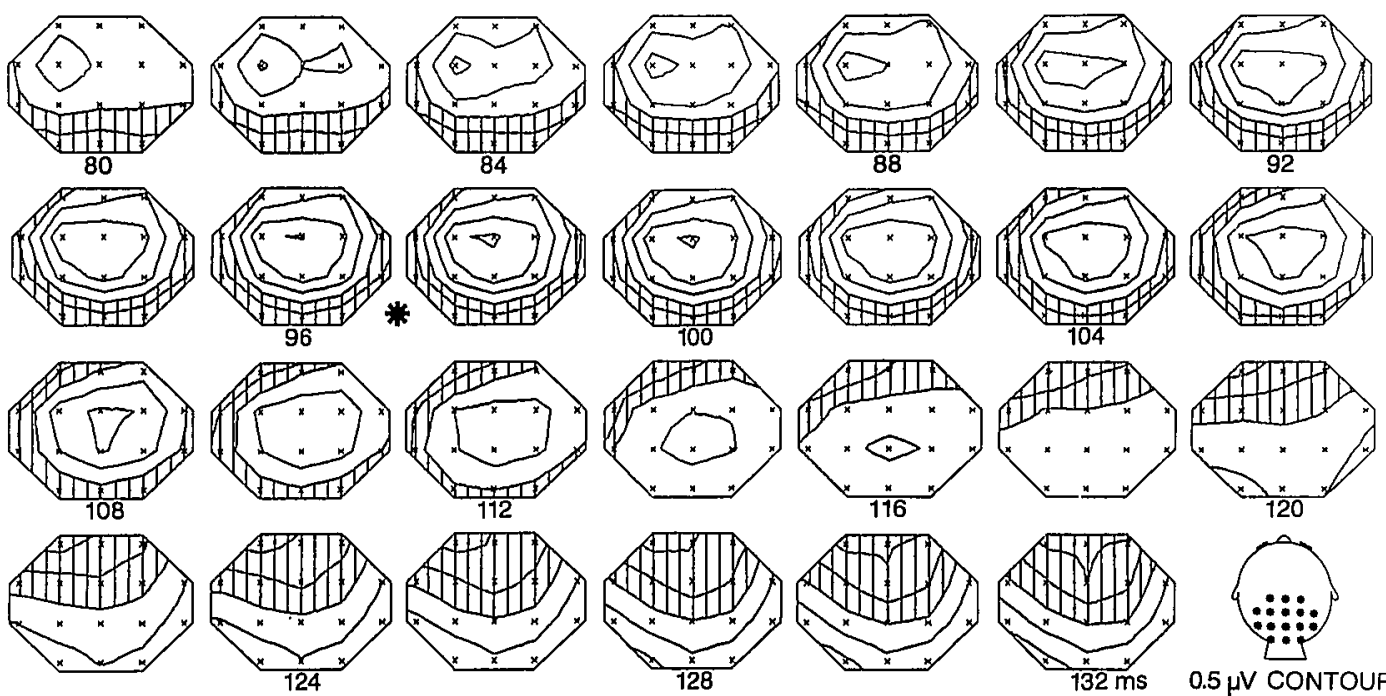

$0.5 \mu \vee$ CONTOURS

Fig. 23. Series of grand mean potential field maps evoked by upper hemiretinal stimuli between 80 and $132 \mathrm{~ms}$ at $2 \mathrm{~ms}$-intervals (hatched areas negative with respect to the average reference). Asterisk indicates time of maximal global field power. Same data as Fig. 22. (From Skrandies 1986a: by permission of Butterworth Publishers, Stoneham) 


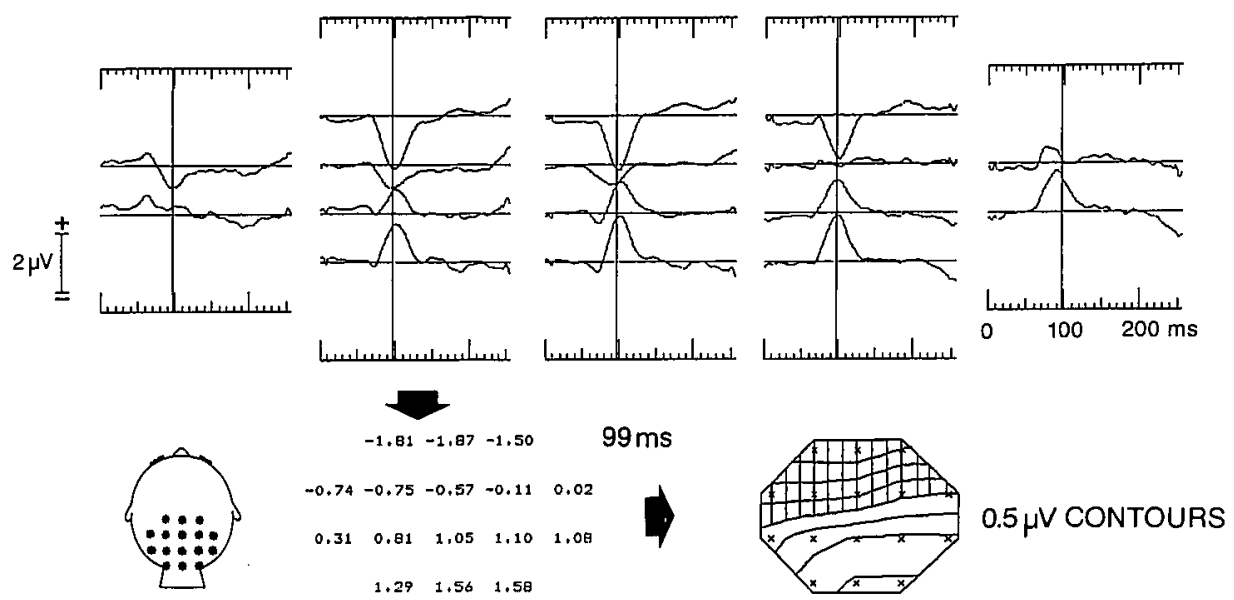

Fig. 24. Grand mean evoked potential waveshapes of 11 subjects evoked by lower hemiretinal stimulation. As in Fig. 22, the construction of a potential map at component latency is illustrated. (From Skrandies 1986a: by permission of Butterworth Publishers, Stoneham)
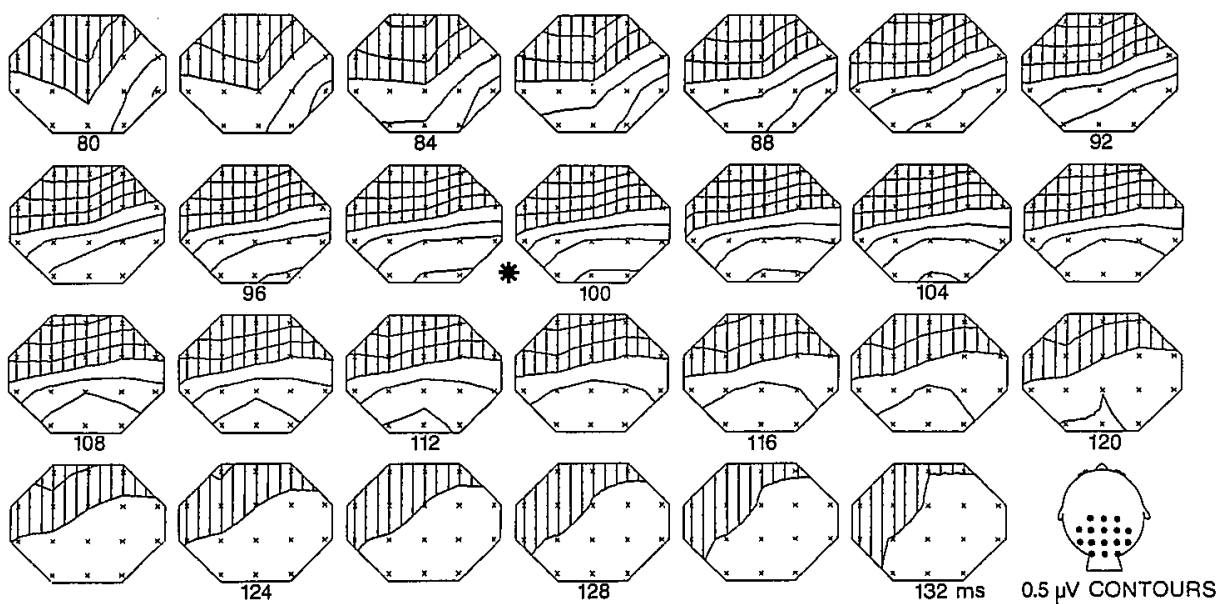

Fig. 25. Series of grand mean potential field maps evoked by lower hemiretinal stimuli between 80 and $132 \mathrm{~ms}$ at $2 \mathrm{~ms}$-intervals (hatched areas negative with respect to the average reference). Asterisk indicates time of maximal global field power. Same data as Fig. 24. (From Skrandies 1986a: by permission of Butterworth Publishers, Stoneham)

Fig. 26. Global field power of grand mean data of Figs. 23 and 24 in 11 subjects. Field power values are scaled to $100 \%$. Note that the maximum occurs earlier for upper than lower hemiretinal stimuli

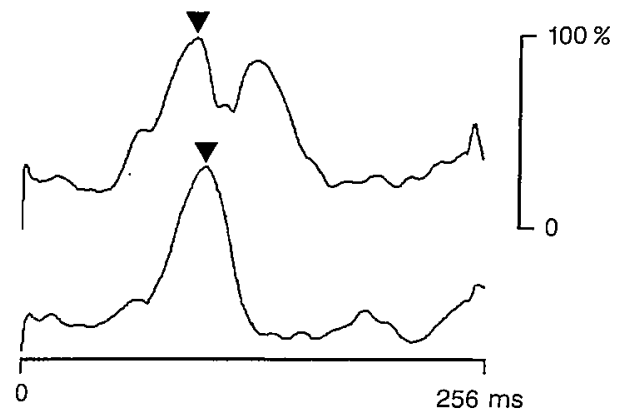
(see arrowheads)

LOWER HR

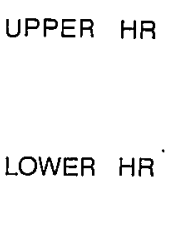

$256 \mathrm{~ms}$ 


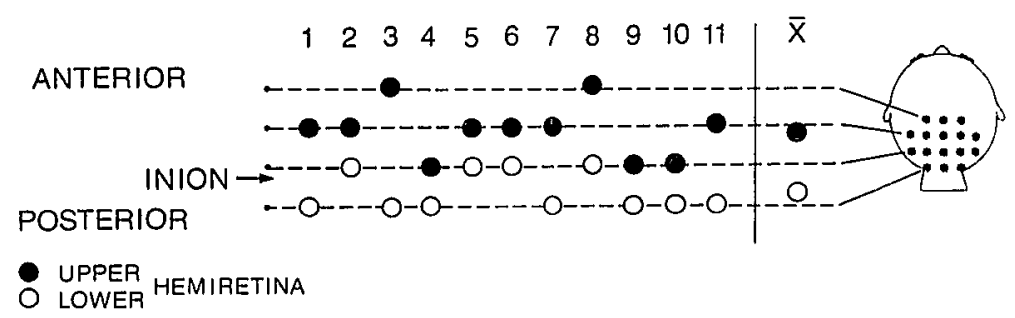

Fig. 27. Distribution of potential maxima in the anterior-posterior direction of 11 subjects at individual component latencies for upper and lower hemiretinal grating stimuli, and mean locations (right). The location differences are statistically significant $(t=7.45$, $p<0.0005$ ). Individual data of Figs. 23 and 25 were used. Electrode positions as indicated on the right

Component location differences were also tested using the individual data as shown in Fig. 27, where component locations evoked by upper of lower hemiretinal stimuli are illustrated in the anterior - posterior direction. The mean location difference was $4.62 \mathrm{~cm}(t=7.45, p<0.0005)$.

\subsection{Implications for the Clinical Use of Visual Evoked Potentials ${ }^{2}$}

The conventional recording of human sensory evoked potentials is a noninvasive. method with a time resolution in the order of milliseconds and a high sensitivity to sensory stimulus parameters. Since the intracranial electrical fields spread instantaneously via volume conduction, the latency of evoked potential components gives unambiguous information in the time domain. Analysis of evoked potential latency is considered a useful measure for diagnostic purposes. In the normal population, the most replicable VEP component is the P100 component, with a latency of approximately $100 \mathrm{~ms}$ and a small standard deviation of only between 3 and $6 \mathrm{~ms}$. (Of course, both mean and standard deviation depend on the exact physical stimulus parameters.) Owing to this low variability, latency measures can be employed for detecting pathological cases. A major application of evoked potential recording in the visual modality is the establishment of clinically silent damage to the optic nerve in patients suspected of multiple sclerosis (Halliday et al. 1973). Latency prolongation of only a few milliseconds may identify a patient's result as pathological. However, such latency differences may also be caused by the existing physiological differences between the upper and lower retinal systems, as I have shown above. Therefore, it appeared of interest to investigate whether the differences are relevant for diagnostic conclusions in a clinical setting.

325 unselected neurological patients suspected to have multiple sclerosis (i.e., "possible" and "probable" cases according to the scale of McAlpine et al. 1972) were investigated. The only criteria for inclusion in the study were visual acuity

2 Patient data were obtained from routine examinations at the Neurology Department of the University Hospital, Zürich. The permission to use the data is gratefully acknowledged 
above 0.8 and reliable evoked potential recordings. This yielded VEP data from 512 eyes of 325 patients.

Evoked potentials were recorded from an array of four electrodes equidistantly spaced in the midline between $2 \mathrm{~cm}$ below and $7 \mathrm{~cm}$ above the inion referred to a frontal electrode at Fz. Checkerboard reversal stimuli of $96 \%$ contrast $\left(60^{\prime}\right.$ arc check size) were presented as a circular target of $15.5^{\circ}$ arc diameter at a distance of $90 \mathrm{~cm}$ to the upper or lower hemiretina. Repeated runs were obtained of each condition, and component latency was determined by the occurrence time of the maximal potential range within the recording array.

Patients were classified as pathological when one or both eyes had component latencies exceeding the normal range, defined as latency values above the mean plus three standard deviations of a group of healthy controls investigated earlier (Lehmann and Mir 1976).

The patients were classified according to various combinations of upper and/or lower retinal component latencies. The numbers and percentages of pathological and normal cases depended on whether only upper, only lower, both upper and lower, or either upper or lower evoked potential latencies were considered:

1. When either upper or lower retinal evoked potential latencies were taken as criterion of normality, 257 of the patients $(79.07 \%)$ had to be classified as pathological.

2. Only 68 patients $(20.92 \%)$ showed normal latencies for both upper and lower retinal stimulation.

3. In all cases where the upper hemiretinal stimuli showed prolonged latencies, the lower hemiretinal components were also abnormal.

4. Following upper retinal stimulation, in only 69 patients $(21.23 \%)$ did the components evoked exceed the normal latency range, and 256 patients $(78.76 \%)$ had normal latencies for both eyes. These data will be further analyzed in detail below (see Fig. 28).

5. In $188(73.44 \%)$ of the 256 patients with normal upper hemiretinal latencies, the lower hemiretinal evoked component latencies were pathological, while the classification of 68 patients $(26.56 \%)$ remained normal.

Figure 28 shows the frequency distribution of component latencies in the two stimulus conditions of the 256 patients for whom normal latencies were obtained in both eyes with upper retinal stimulation. The latency values of the lower retina are shifted toward longer latencies, and they have a much broader distribution than upper hemiretinal latencies. The upper retina yielded component latencies with a mean of $100.5 \mathrm{~ms}$ and a standard deviation of $5.2 \mathrm{~ms}$, whereas components evoked by the lower retina had a mean of $118.1 \mathrm{~ms}$ and a standard deviation of $14.5 \mathrm{~ms}$. Paired $\mathrm{t}$-tests confirmed that the latency differences were highly significant $(p<0.0001)$.

In addition, upper retinal stimuli resulted in normally distributed latencies, while the latency values of the lower retinal stimuli appeared to deviate from a Gaussian distribution (Fig. 28). The distribution parameters skewness and kurtosis were computed and found to differ for the two stimulus conditions. The upper retinal data were not significantly different from a normal distribution (skewness $-0.163, z=1.51$, n.s.; kurtosis $-0.257, z=1.19$, n.s.) whereas the lower retinal data deviated from normality (skewness 1.259, $z=11.66, p<0.0001$; kurtosis 


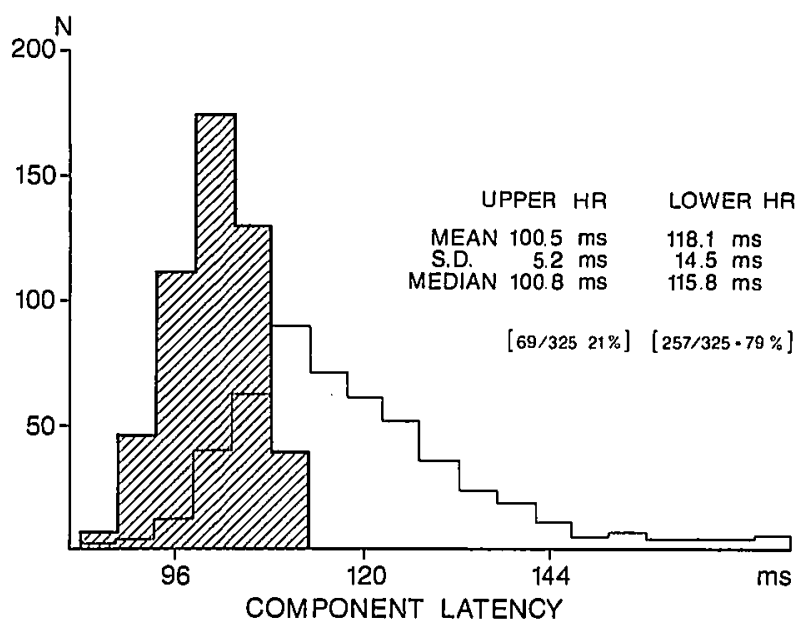

Fig. 28. Frequency distribution of component latencies of 256 patients in which normal latencies were found for upper retinal stimulation of both eyes. The latency values of the lower hemiretina (thin line) show a broader distribution than upper hemiretinal latencies (thick line). Mean, standard deviation, and median values of the population as indicated. The data obtained for the upper retina are not significantly different from a normal distribution, while the lower retina data deviate from normality (see test)

2.621, $z=12.16, p<0.0001)$. These differences were tested by the procedure described by van der Waerden (1971, pp. 229-230).

In summary, the data of a population of unselected neurological patients show impressively how inherent physiological differences between the two hemiretinal systems increases the variability of evoked potential measures. The high intersubject variation of component latencies evoked by the lower hemiretina suggests that this stimulus condition is not at all appropriate for diagnostic purposes: Because of the large standard deviation, which is nearly three times that of the upper hemiretina, the normal range becomes very large. When lower instead of upper retinal stimuli were used, the discriminative power between normal and pathological results decreased and the number of false-negative increased (i.e., pathological cases become classified as normal).

More importantly, the physiological differences as reflected by different component latencies may cause a false pathological diagnosis if retinal stimulus location is not taken into account. A simple change of fixation toward the lower parts of the stimulus display is followed by prolonged component latencies. Typically, the stimulus displays used are not much larger than $20^{\circ}$ and "wrong" fixations may easily occur. In our data the number of pathological latencies increases from 69 to 257 patients (i.e., by $57.84 \%$ ) when the lower retinal data are used. For clinical purposes it appears of major importance to carefully control the patients' fixations. The upper hemiretinal responses yield more stable results with less intersubject variability and thus prove more useful for meaningful diagnostic interpretations. 


\section{Electrophysiological Differences at Other Stages of the Visual System}

In the preceding section evidence was obtained that stimuli presented to the upper hemiretinal system are processed more efficiently than those presented to the lower hemiretinal system. The difference is reflected in shorter latencies of the cortical evoked components. In this section I will investigate whether such differences can already be detected at more peripheral parts of the visual system. The electro-oculogram (EOG) can be used as an electrophysiological method to study changes of the intraocular standing potential of the eye which shows a long latency response to the onset of steady illumination. This potential is believed to reflect the rate of metabolism elicited by light stimuli (Arden and Kelsey 1962b; Marmor and Lurie 1979), and the use of small localized test lights may reveal local differences at the first step of visual information processing (Sect. 4.1).

In electroretinography (ERG) contrast alternating patterns can be used as localized retinal stimuli which elicit the so-called pattern ERG. The pattern ERG is believed to originate from retinal structures associated with the ganglion cell layer, and the experiments presented below will demonstrate differences between upper and lower retinal areas reflected in the human pattern ERG (Sect. 4.2).

\subsection{Retinal Pigment Epithelium Activity ${ }^{3}$}

Eye movements can be electrically recorded from skin electrodes placed at the canthi of the eye because the vertebrate eye forms an electrical dipole with the cornea positive with respect to the fundus. This is the electrophysiological basis of eye movement recordings (EOG). The EOG can be used as an indirect method to study changes of the intraocular potential when eye movements of a constant amplitude are made (Arden and Kelsey 1962a, b). The short-latency a-, b-, and cwaves of the ERG [in the order of milliseconds, or seconds in case of the c-wave (Granit 1947; Armington 1974)] are elicited by the onset of steady illumination of the eye, and a large positive deflection, called the "light peak," occurs only after several minutes. Investigations of human patients (Arden and Kelsey 1962b; Täumer 1976), show that the retinal pigment epithelium is necessary to produce the light peak, which is interpreted as an indicator of the rate of metabolism (Marmor and Lurie 1979). Valeton and van Norren (1982), Linsenmeyer and Steinberg (1982), and Griff and Steinberg (1982) performed intraocular microelectrode recordings in various species, and their data suggest that the source of the light peak is located in the basal membranes of the retinal pigment epithelium. The standing potential of the eye is recorded in man indirectly by the EOG, and its covariation with stimulus parameters allows conclusions to be drawn about the functional interaction between the pigment epithelium and the retinal photoreceptor layer under different experimental conditions.

In the experiments presented in this section, the EOG was used in order to investigate whether electrophysiological differences between the upper and lower retinal areas are restricted to evoked potential measures (see Sect. 3), or whether differences can be detected already at a very basic retinal level. The activity of the

\footnotetext{
${ }^{3}$ Results of this section have been published elsewhere (Skrandies and Baier 1986)
} 
pigment epithelium as quantified by EOG measurements of perifoveal upper and lower retinal regions will be described. Absolute differences in response to a small localized light stimulus, as well as the different reactivity of upper and lower retinal areas to changes of the test light intensity, will be reported.

Twelve healthy subjects between 19 and 32 years of age participated in this study. The measurements extended over five different sessions lasting about $2 \mathrm{~h}$ each, and in order to minimize diurnal variations all experiments were performed at the same time of day (late in the afternoon). The pupils were dilated maximally by the application of Mydriaticum Roche ${ }^{\circledR}$ and Neosynephrine ${ }^{\circledR}$, and Hellige Ag-AgCl recording electrodes were fastened at the lateral and medial canthi of each eye.

During an adaptation period of at least $60 \mathrm{~min}$, the subject was exposed to a white adaptation light of $-1.77 \mathrm{log}$ candela $/ \mathrm{m}^{2}$ which was projected as a Ganzfeld onto a half-cylindric acrylic screen. After about $1 \mathrm{~h}$ the stability of the baseline was tested. The subject performed at 1-min intervals horizontal eye movements with an excursion of $40^{\circ}$. About $8 \mathrm{~min}$ later the standing potential (i.e., the EOG amplitude) had reached a steady state, and a circular white test light of $10^{\circ}$ diameter with a luminance of $2.73 \mathrm{log}$ candela $/ \mathrm{m}^{2}$ was back-projected at the center of the Ganzfeld. The adaptation light remained on throughout the session. Foveal, and peripheral upper and lower retinal areas at $10^{\circ}$ and $40^{\circ}$ eccentricity along the vertical meridian were stimulated in different experimental sessions in a quasi-randomized order by changing the location of fixation marks.

The EOG was recorded with a "Tönnies-EOG-System" consisting of a DC amplifier (low pass at $20 \mathrm{~Hz}$ ), and an analog computer which integrated the amplitudes of ten consecutive eye movements. The raw EOGs and the integrated values were plotted online on an $x-y$ plotter. A baseline was defined as the mean amplitude of the last eight recordings of the adaptation period.

In order to discriminate the light peak from spontaneous fluctuations of the dark value, the $95 \%$ confidence limit of the values obtained in the adaptation period was determined for each eye separately using the $t$-distribution. Peak values exceeding this confidence limit were considered reliable, and only two of the 120 measures obtained ( 12 subjects $\times 2$ eyes $\times 5$ retinal locations) had to be excluded. The light peak amplitudes were expressed as percentages of the adaptation values, and the means of the right and left eye of each subject were used for further analysis. Because percentage values are not normally distributed, Wilcoxon tests were used for comparing experimental conditions.

An additional series of experiments was performed with three subjects, each of whom participated in 15 sessions. In these experiments we investigated the reactivity of upper and lower retinal areas to different test light intensities. The same procedure as described above was used, but the measurements were restricted to upper and lower retinal areas at $10^{\circ}$ eccentricity. The test light intensities varied between 2.42 and $3.63 \mathrm{log}$ candela $/ \mathrm{m}^{2}$ in steps of $0.5 \mathrm{log}$ candela $/ \mathrm{m}^{2}$. This yielded five light peak values for each location, and the relationship between EOG amplitudes and light intensity was established by computing linear regressions.

The original EOGs of one subject measured at five different retinal locations are illustrated in Fig. 29A. About $10 \mathrm{~min}$ after the light onset the light peak occurs in all recordings with a mean peak latency of $9.5 \mathrm{~min}$ in the subject population. The latencies were similar for all retinal locations, and there were also no significant differences between central and peripheral light peak latencies. 
A
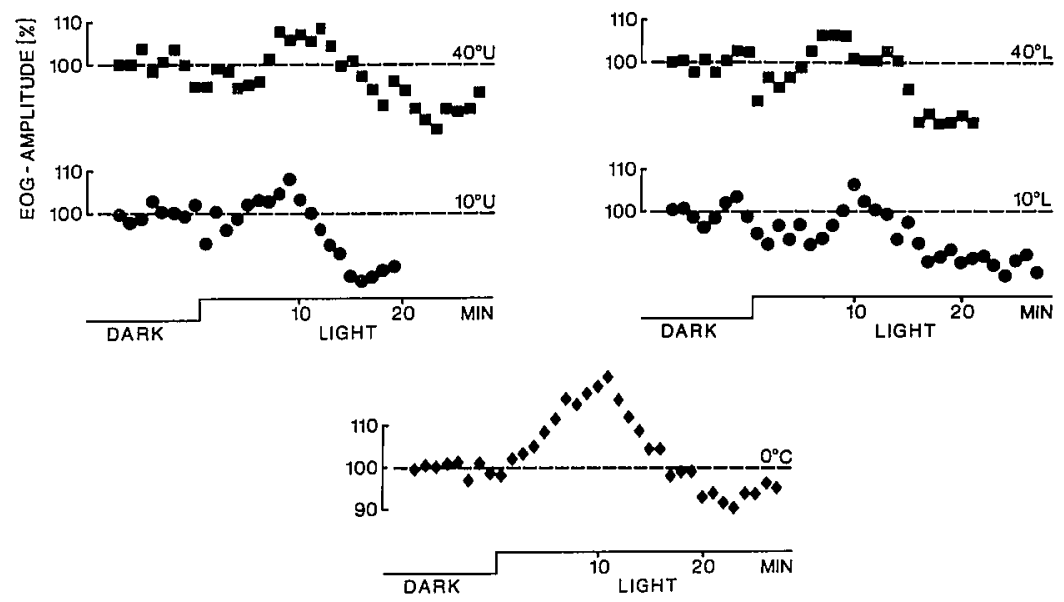

B

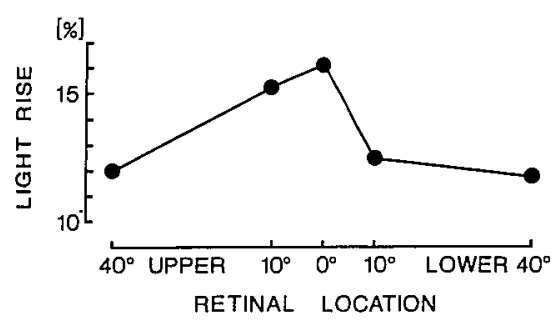

Fig. 29. A Original local EOG amplitudes of one subject obtained at various retinal locations along the vertical meridian. Retinal locations refer to the center of the $10^{\circ}$ arc test light and range from $40^{\circ}$ on the upper retina $\left(40^{\circ} \mathrm{U}\right)$ via $10^{\circ}$ on the upper retina $\left(10^{\circ} \mathrm{U}\right)$, the center $\left(0^{\circ} \mathrm{C}\right)$, and $10^{\circ}$ on the lower retina $\left(10^{\circ} \mathrm{L}\right)$ to $40^{\circ}$ on the lower retina $\left(40^{\circ} \mathrm{L}\right)$. A rise in potential peaking after approximately $10 \mathrm{~min}$ was measured with reference to the base value, which was set to $100 \%$. Note that there are no differences in latency, but differences in amplitudes of the EOGs (see text). B Distribution of local EOG amplitudes in a population of 12 subjects. Median values are presented: the differences between the center and the periphery, as well as the differences between upper and lower areas at $10^{\circ}$ eccentricity, were statistically significant. (After Skrandies and Baier 1986)

The light peak amplitudes were in the order of only $10 \%-20 \%$ above the baseline condition. This is accounted for by the small test light used. The highest amplitudes of the light peak occurred after central stimulation (median over all subjects: $16.12 \%$ ), and central light peak amplitude increases were significantly larger than peripheral light peak amplitude increases (median difference $2.47 \%$, $p<0.05$ ). Perifoveal upper retinal areas had higher amplitudes of the light peak than the corresponding lower retinal areas. However, these differences were significant only at $10^{\circ}$ eccentricity, where upper retinal areas showed a median light peak amplitude rise of $15.26 \%$ while lower retinal areas had a median light peak of $12.44 \%$ (median difference $2.82 \%, p<0.025$ ). At $40^{\circ}$ eccentricity the same 
tendency was found $(12.06 \%$ light peak amplitude for the upper, $11.76 \%$ for lower retinal areas), but due to the large intersubject variation the difference was not statistically significant (median difference $0.30 \%$, n.s.). The difference between the results obtained at $10^{\circ}$ and at $40^{\circ}$ eccentricity may be explained by the fact that rod densities are much higher in perifoveal areas than farther out in the retinal periphery (Østerberg 1935). This may well account for the higher variability observed at $40^{\circ}$ eccentricity.

The population data are summarized in Fig. 29B showing the distribution of the median light peak amplitudes at the five retinal locations examined. The upper retinal areas have higher amplitudes than the corresponding lower retinal areas, and central stimuli yield the highest amplitudes.

With increasing intensity of the test light the light peak amplitudes increased, and a linear relationship was observed in all subjects. The mean correlation coefficient between EOG amplitudes and the logarithm of the test light intensities was 0.75 . Figure 30 illustrates the slopes of the regression lines computed individually for each subject for upper and for lower retinal stimuli. In order to enable a direct comparison between retinal locations independent of the absolute EOG amplitude differences, the regression lines were normalized, and Fig. 30 shows the light peak amplitudes relative to the amplitudes found with a test light intensity of 2.5 $\log$ candela $/ \mathrm{m}^{2}$. Upper retinal stimuli consistently elicit steeper slopes than lower retinal stimuli. This suggests a greater sensitivity of the upper retinal areas to changes in light intensity.

Light peak amplitude increases in the order of less than $20 \%$ were also reported in other EOG studies in which small localized test stimuli were used (Aschoff

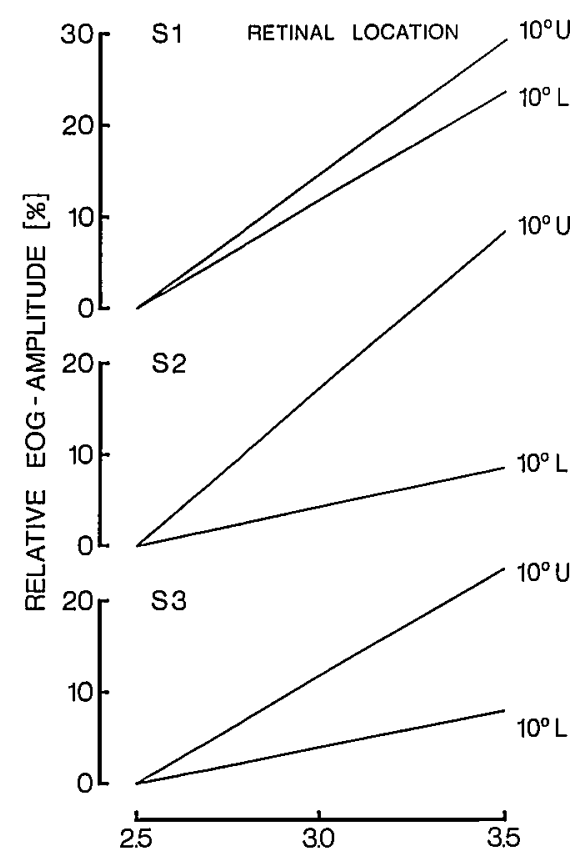

TEST LIGHT INTENSITY [LOG CO/M²]
Fig. 30. Slopes of regression lines of three subjects indicating the relationship between relative EOG amplitude and the logarithm of the test light intensity. Stimuli presented $10^{\circ}$ above the fovea $\left(10^{\circ} \mathrm{U}\right)$ yield steeper slopes than stimuli $10^{\circ}$ below the fovea $\left(10^{\circ} \mathrm{L}\right)$. Data were obtained at five light intensities between 2.42 and $3.63 \mathrm{log}$ candela $/ \mathrm{m}^{2}$ with intervals of $0.5 \mathrm{log}$ candela $/ \mathrm{m}^{2}$. In order to enable a direct comparison between upper and lower retinal responses independent of the absolute EOG amplitudes, the values recorded at $2.5 \mathrm{log}$ candela $/ \mathrm{m}^{2}$ were set to 0\%. (After Skrandies and Baier 1986) 
1981). The finding of slightly, but statistically significantly higher EOG light peak amplitudes for foveal than perifoveal stimuli, in the order of $2.5 \%$, confirms reports on the contribution of cone activity to the light peak amplitude (Afanador and Andrews 1978). This interpretation is in line with data obtained from congenital achromats (Elenius and Aantaa 1973), and similar differences between EOG light peak amplitudes elicited by central or peripheral stimuli were observed by Aschoff (1981) and Dodt and Baier (1984). The differences between the upper and lower perifoveal retinal areas at $10^{\circ}$ eccentricity were small but consistent and statistically significant in the subject population. At $40^{\circ}$ eccentricity similar differences were observed but the variability of the EOG amplitudes was high, and the differences between upper and lower retinal stimuli did not achieve statistical significance. The smaller variability of the data at $10^{\circ}$ eccentricity is explained by the fact that a larger number of photoreceptors are activated by a test light located between $5^{\circ}$ and $15^{\circ}$ eccentricity than by stimuli located between $35^{\circ}$ and $45^{\circ}$ eccentricity (see $\emptyset$ sterberg 1935 ).

The systematic differences of light peak amplitudes cannot be caused by intrasubject variation in the various test sessions. In addition, variation of electrode placement or electrode impedance, which may cause different absolute EOG amplitudes, cannot account for the effects of retinal location in our data, because the light peak amplitudes were always referred to the EOG values obtained in the baseline condition at the end of the respective dark adaptation periods.

In addition to the absolute light peak amplitude differences there were also relative differences in amplitude change when the test light intensity was varied. As expected from the results of Arden and Kelsey (1962b), the light peak amplitude was proportional to the logarithm of the test light intensity. Upper retinal areas consistently showed a larger increase of the EOG response, which suggests that the upper retina is more sensitive to changes of light intensity.

From clinical studies it is known that the response of the standing potential to light requires contact between the photoreceptors and the pigment epithelium, and thus the light peak is believed to reflect the rate of metabolism elicited by light stimuli (Arden and Kelsey 1962b; Marmor and Lurie 1979). The light peak presumably originates in the basal membranes of the pigment epithelium (Valeton and van Norren 1982; Linsenmeyer and Steinberg 1982; Griff and Steinberg 1982). The finding that stimulation of upper retinal areas at $10^{\circ}$ eccentricity is followed by significantly larger light peak amplitudes than stimulation of lower retinal areas suggests that stimuli presented to upper retinal areas elicit a higher level of light-induced activity, reflecting the interaction between the photoreceptors and the retinal pigment epithelium, than the stimulation of the corresponding lower retinal areas. Anatomical differences in the regional distribution of rods and cones in the human retina, where a higher density of photoreceptors is reported for upper retinal areas (Østerberg 1935), support this interpretation. The amplitude of the light peak can be interpreted as reflecting the rate of metabolism elicited by light stimuli, and thus should correlate with the anatomically determined photoreceptor density (see also Sect. 6.1.2).

In summary, the EOG data show that absolute and relative differences between upper and lower retinal areas in response to light stimuli can be found at the first step in visual information processing at a very basic retinal level. 


\subsection{Human Pattern Electroretinograms}

Contrast alternating patterns produce no stray-light artifacts and have thus been used as localized retinal stimuli in ERG studies (Riggs et al. 1964; Armington 1968). Such stimuli elicit the so-called pattern ERG that can be recorded from the intact human eye. The origin of the components of the pattern ERG is different from that of those of the luminance ERG, which reflects the activity of the retinal layer of photoreceptors (a-wave) and the activity of bipolar and Müller cells (bwave). While the exact generating mechanisms of the components of the pattern ERG remain a matter of discussion (cf Riemslag et al. 1985), this experimental technique can certainly be employed to investigate potentials generated at a higher retinal level than the pigment epithelium activity described in Sect. 4.1.

There is some evidence that the pattern ERG originates from retinal structures associated with the ganglion cell layer. Maffei and Fiorentini (1981) and Maffei (1982) have shown that the pattern ERG of the cat diminishes gradually after transection of the optic nerve and eventually disappears after about 4 months, while the luminance ERG remains unchanged. Holläncer et al. (1984) confirmed these findings and correlated the attenuation of the pattern ERG responses with the retrograde changes in retinal morphology following the section of the optic nerve. These authors demonstrated that their electrophysiological findings were paralleled by morphological changes restricted to the nerve fiber and ganglion cell layers of the retina. Similar pattern ERG findings in the monkey after sectioning the optic nerve were reported by Maffei et al. (1985).

Clinical observations support this interpretation: Groneberg and Teping (1980) described pattern ERG recordings from a patient with a traumatic lesion of the optic nerve. Shortly after the lesion both pattern and luminance ERGs could be recorded, whereas about 3 months later the pattern ERG had disappeared while the luminance ERG remained unchanged.

The experiments presented in this section were aimed at demonstrating differences between upper and lower retinal areas, reflected in the human pattern ERG, which may be associated with the activity of retinal ganglion cells.

In order to arrive at meaningful conclusions from pattern ERG recordings in man, it appeared of major importance first to establish that the electrical activity recorded (i.e., the components of the pattern ERG) is of retinal origin. Surprising results were reported from experiments where pattern ERGs were recorded from an eye which was occluded while the contralateral eye was stimulated (Seiple and Siegel 1983). Following the considerations on the scalp topography of electrical fields outlined in Sect. 2, a reference-independent measurement and analysis strategy was applied to simultaneous recordings of evoked potentials and pattern ERGs. In Sect. 4.2.2 experiments will be reported in which significant differences between upper and lower retinal areas were found using pattern ERG recordings.

\subsection{Spatial Distribution of Pattern Electroretinograms and Evoked Potentials}

In order to prove that the pattern ERG has its origin in the eye and is not a farfield potential originating in the visual cortex, simultaneous multichannel recordings were made at the eye and at several scalp locations. Gold foil electrodes were 
Fig. 31. Potential waveshapes recorded from a row of eight electrodes covering laterally the distance between the left eye and the inion (see head schema). Reference electrode $(R)$ at the vertex $(\mathrm{Cz})$. Checkerboard reversal stimuli evoked a pattern ERG around $60 \mathrm{~ms}$ in the eye channel and VEP activity around $100 \mathrm{~ms}$ in the occipital channels
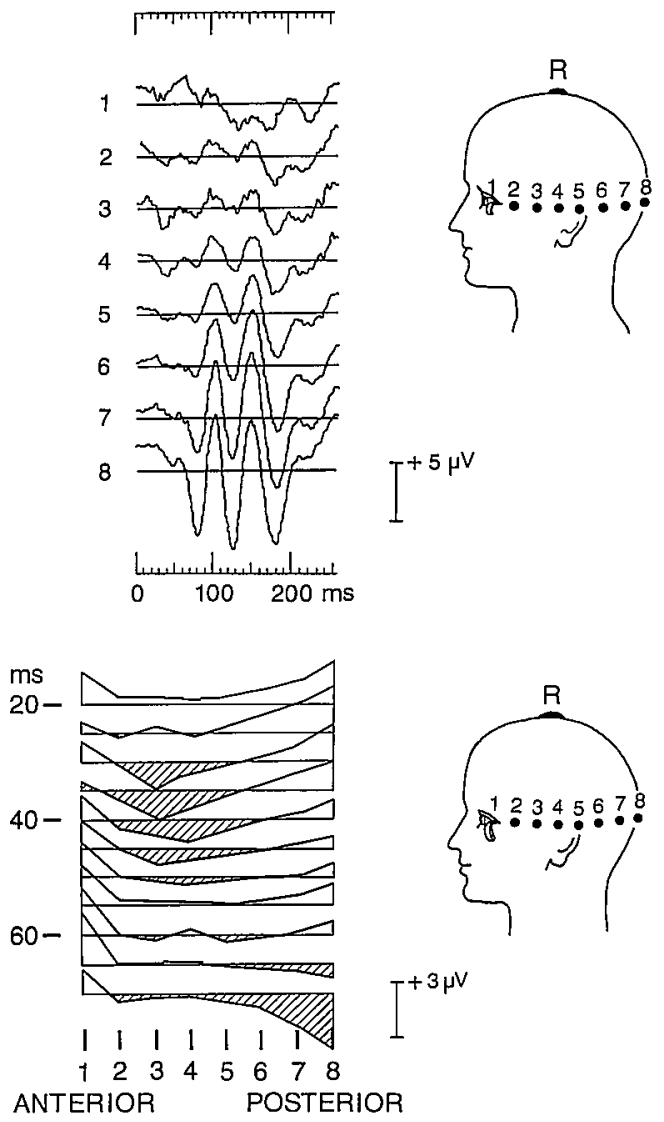

Fig. 32. Data of Fig. 31 shown as potential profiles along the head between 20 and $70 \mathrm{~ms}$ latency. Note the consistent shape of the anterior potential gradients

used to record the pattern ERG from the eye (Arden et al. 1979). Two different scalp electrode montages were used. In one experiment, eight recording electrodes were placed in a line along the side of the head from the left eye to the inion with a vertex reference (Fig. 31). In a second topographical experiment, eight recording electrodes were spaced equidistantly from the eye via the forehead along the midline of the head to the inion using the ear as reference point (Fig. 33). The reference sites were selected merely for convenience in order to enable waveform comparisons of the pattern ERG with the published literature; they had no consequence for the analysis of the electrical field distribution (Sect. 2).

A topographical distribution with large potential gradients around the eye is expected for retinal potentials, whereas visual cortical sources produce steep potential gradients over the occipital areas. Figure 31 shows the potential waveshapes recorded to a checkerboard reversal stimulus (check size $30^{\prime}$ arc, $80 \%$ contrast) fixated centrally. At early latency times, a positive deflection appears after about $60 \mathrm{~ms}$ in the first channel while the other recording channels show little activity. Around $100 \mathrm{~ms}$ on the other hand, waveform peaks occur in all recording channels, and the positivities are most pronounced over the occipital scalp areas. The topographical changes over time along this lateral row of electrodes are illustrated in Fig. 32 as a series of potential profiles. Between 40 and $70 \mathrm{~ms}$ a potential peak 


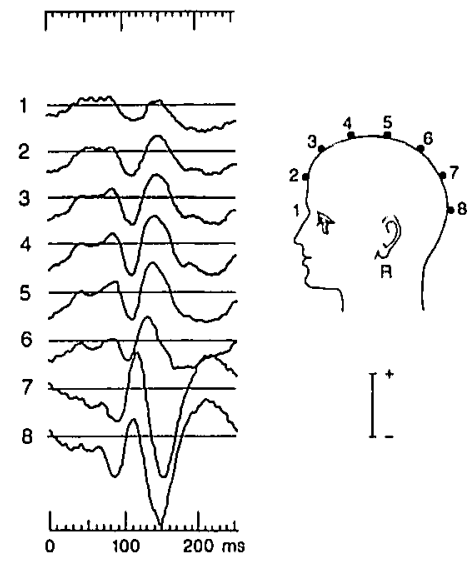

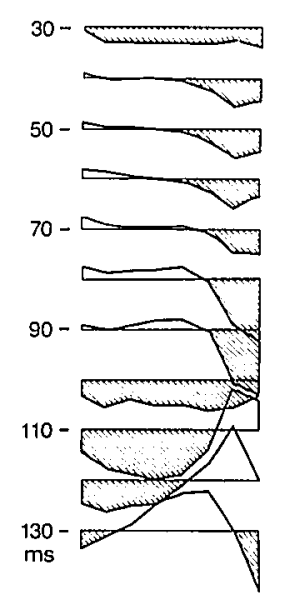

Fig. 33. Potential waveshapes and scalp potential distributions evoked by a checkerboard reversal stimulus presented to the right eye. The left eye was occluded, and activity was recorded in eight channels along the midline between the left eye and the inion using a reference electrode $(R)$ at the ear. Channels $1-6$ are on nearly equipotential levels (potential profiles on the right) while occipital recording channels show VEP activity after $100 \mathrm{~ms}$.

Calibration: $5 \mu \mathrm{V}$

builds up at the eye electrode, reaching its maximum at $65 \mathrm{~ms}$. This early latency range is dominated by a steep potential gradient between the eye electrode and the second electrode, whereas the potential gradients between channels 2 and 6 are shallow. The gradients over the occiput (between channels 7 and 8 ) show less consistent behavior during these early latency times: between 20 and $60 \mathrm{~ms}$ the gradient is first positive, and then becomes negative. In contrast, the anterior potential gradient remains negative throughout the analysis period considered in Fig. 32. These differences between the anterior and the occipital recording channels suggest that the source of the evoked activity recorded in the first channel as a pattern ERG is located in the eye.

Similar conclusions arise from data obtained from a different subject with a midline electrode montage along the head from the left eye via the forehead to the inion. in these experiments the effect of occluding the recorded eye was also investigated. Seiple and Siegel (1983) had reported that a pattern ERG waveform can be recorded even when only the fellow eye is stimulated. We repeated this stimulus condition: Fig. 33 shows the data obtained when the right eye was presented a checkerboard reversal stimulus while the ERG activity was recorded from a row of eight midline electrodes equidistantly spaced between the left eye and the inion. The anterior electrodes of the array appear to be on nearly equipotential levels, whereas in the posterior recording channels the occipitally originating evoked potential activity is seen. The corresponding potential profiles are shown in Fig. 33 on the right. In the anterior channels, small potential gradients are seen throughout the analysis period. Occipitally, a relative negativity dominates the times around $80 \mathrm{~ms}$, and at $110 \mathrm{~ms}$ the scalp distribution shows an occipital positivity surrounded by steep gradients. This scalp topography is similar to that illustrated before for components evoked by checkerboard reversal stimuli (see potential waveshapes and profile in Fig. 5, map series in Fig. 10, profile series in Fig. 18). From these topographical multichannel data it is obvious that the waveshape recorded at the eye cannot be interpreted as a pattern ERG. The scalp distribution evoked by the same checkerboard reversal stimulus presented to the left eye is illustrated in Fig. 34. The potential waveshapes are similar to those evoked by stimulation of the right eye except for the electrical ac- 
Fig. 34. Potential waveshapes and scalp potential distributions evoked by a checkerboard reversal stimulus presented to the left eye. Electrical activity was recorded in eight channels along the midline between the left eye and the inion. Channels 2 through 8 show a similar pattern of activity throughout the recording period, as in Fig. 33. The eye channel displays a pattern ERG around $50 \mathrm{~ms}$ resulting in steep anterior potential gradients. Same subject as in Fig. 33. Calibration: $5 \mu \mathrm{V}$

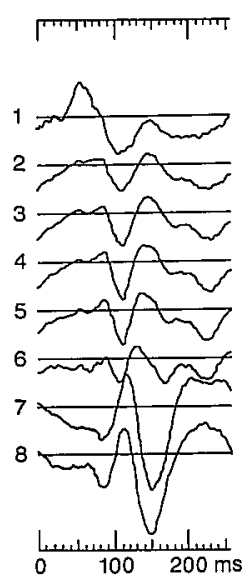

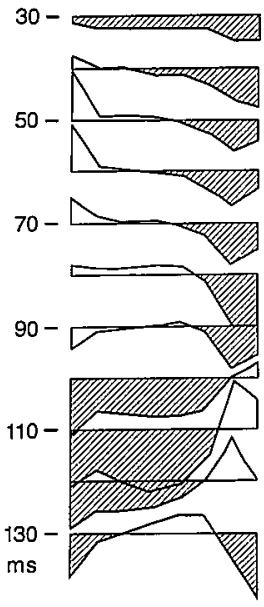

tivity recorded in the first channel, which is reminiscent of the pattern ERG. The potential profiles on the right of Fig. 34 around $50 \mathrm{~ms}$ have steep gradients anteriorly (i.e., between the eye electrode and electrode 2) while around $110 \mathrm{~ms}$ there is the same occipital positivity as in Fig. 33.

The topographical analyses of the pattern ERG and VEP show that retinal activity can be meaningfully interpreted and distinguished from contamination of cortical sources by considering electrical scalp field distributions. For examining upper and lower retinal areas, similar multichannel recordings were obtained, and the latency of the pattern ERG components was determined by the occurrence of a maximal potential gradient at electrodes at and near the eye. This method is independent of the reference electrode, and it avoids confusion generated by waveshape analysis (Seiple and Siegel 1983).

\subsubsection{Differences Between Upper and Lower Retinal Areas}

Simultaneous recordings of pattern ERGs and potentials evoked by central, upper, or lower retinal stimuli were obtained in a population of 11 healthy adult subjects.

VEPs were recorded from six electrodes spaced equidistantly along the midline between $40 \%$ of the nasion-inion distance above the inion and an electrode $10 \%$ below the inion. Pattern ERGs were recorded from the left eye using a gold foil electrode (Arden et al. 1979) inserted under the lower lid. Two skin electrodes were placed at the outer canthus of the eye and $3 \mathrm{~cm}$ further posterior. The distances between electrodes 1 and 2 and electrodes 2 and 3 were equal.

The EEG and ERG signals were amplified, bandpassed between 0.1 and $70 \mathrm{~Hz}$, and $A / D$-converted at a rate of 1000 samples per second over a period of $256 \mathrm{~ms}$. In order to avoid artifact contamination of the ERG by eye movements and blinks, 12 averages consisting of only 30-60 sweeps were obtained for each experimental condition in a quasi-randomized order. The data were edited offline, and for final analysis artifact-free grand means of each retinal location were computed for each subject individually. 
Checkerboard patterns consisting of checks of $30^{\prime}$ arc and $62 \%$ contrast were reversed at a rate of 3.9 reversals per second on a TV monitor (rectangular screen of $8.7^{\circ}$ by $11.4^{\circ}$ ) which was rotated by $90^{\circ}$. The subject's head was supported by a chin-forehead rest, and he looked binocularly at fixation marks in the center or at the upper or lower border of the display from a distance of $130 \mathrm{~cm}$.

The vertex electrode served only as recording reference; for analysis of the VEP data the six scalp sites were treated as longitudinal potential profiles, while the pattern ERG data were analyzed as potential profiles along the three electrodes at the eye. Components of the pattern ERG were defined by the maximal potential gradient occurring in a time window between 35 and $75 \mathrm{~ms}$. VEP components were determined by the occurrence times of the maximal potential range which corresponds to the multichannel field power measure between 80 and $140 \mathrm{~ms}$. Three sets of data of a typical subject are illustrated in Fig. 35. The first waveshape shows the pattern ERG, computed as the difference between the eye electrode and the electrode at the outer canthus; the other waveshapes are the scalp channel data which are computed vs the average reference. In all stimulus conditions the pattern ERG has a similar waveshape with a positive component occurring after about 50-60 ms. For central retinal stimuli the pattern ERG amplitude tends to be larger than for upper or lower retinal stimuli. The potential gradients within the three-channel recording array were larger for central stimuli than for peripheral stimuli in all but four of 22 cases.

As shown in Fig. 35 there are also differences in component latency of the maximal gradient of the pattern ERG between experimental conditions, with lower

UPPER

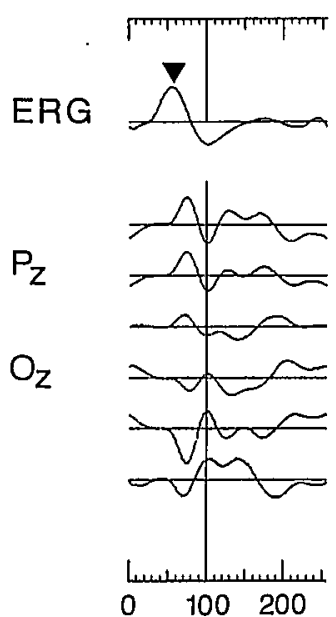

CENTER

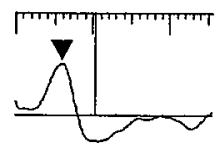

$+4.5 \mu \mathrm{V}$

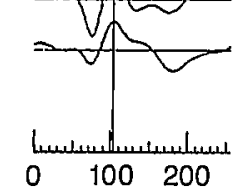

LOWER
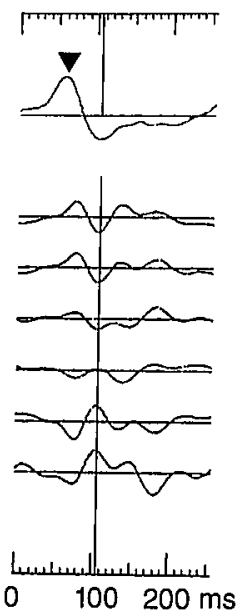

Fig. 35. Pattern ERGs and potential waveshapes evoked by a checkerboard reversal stimulus presented to the upper, the central, or the lower retina. Waveshapes in the first row show the pattern ERGs, computed as the difference between the eye electrode and the electrode at the outer canthus; the other waveshapes are the scalp data which were computed vs the average reference. Vertical lines at times of maximal field power. Note latency differences between upper and lower retinal stimuli in the potential gradients at the eye (component latencies between 50 and $60 \mathrm{~ms}$ ) as well as in the scalp distribution data (component latencies around $100 \mathrm{~ms}$ ) 
retinal stimuli yielding longer latencies than upper retinal stimuli. In the subject population component latency of the pattern ERG was determined independent of the reference electrode by the occurrence of the maximal potential gradient. This resulted in a mean latency of $55.3 \mathrm{~ms}$ for upper retinal stimuli while the responses of the lower retinal areas averaged $59.3 \mathrm{~ms}$ in the subject population. The differences were statistically significant $(t=4.69, p<0.0005)$. The mean component latency evoked by central stimuli was $57.5 \mathrm{~ms}$, and it was different from either upper or lower component latencies only at the $10 \%$ significance level.

The VEP data which had been obtained simultaneously displayed also differences in component latencies as illustrated in Fig. 35. The mean latency difference between components evoked by upper and lower retinal stimulation in the 11 subjects studied averaged $2.0 \mathrm{~ms}$. This comparatively small mean difference might have been caused by the rather large interelectrode distances used and the accordingly crude sampling of the evoked scalp field which contributed to the interindividual variability. However, analysis of the individual data showed that the latency difference was consistent in the subject population, and it achieved statistical significance $(\mathrm{t}=2.19, p<0.005)$.

In summary, the present data show that localized visual stimuli can be used to investigate different parts of the retina by means of ERG recordings. Differences already observed in the interaction between the retinal pigment epithelium and the photoreceptors. (Sect. 4.1) also show up in the electrical responses associated with retinal ganglion cell activity: Stimulation of upper retinal areas yields significantly shorter component latencies of the pattern ERG than stimulation of the corresponding lower retinal areas. While the significant differences observed in the pattern ERG were restricted to the time domain, as reflected by significantly different component latencies, in the EOG recordings the differences were most pronounced in the amplitudes of the light-induced standing potential of the eye. This fact might well be accounted for by the rather crude time resolution of the determination of the light peak of the EOG, which is in the order of minutes, whereas the pattern ERG component latencies are less than $100 \mathrm{~ms}$.

\section{Functional Differences}

The aim in this section is to investigate whether the electrophysiologically determined differences between the upper and the lower retina are paralleled by perceptual differences. Most of the electrophysiological data were discussed in the previous sections mainly in formal terms of amplitudes, latencies, or scalp topography, which do not relate directly to functional aspects of visual perception. Prompted by the evoked potential results, a series of studies was made investigating visual functions such as temporal sensitivity (Sect. 5.1), contrast sensitivity and visual acuity (Sect. 5.2), and discrimination of meaningful alphanumeric stimuli (Sect. 5.3). The results will illustrate consequences on behavioral performance which arise from the physiological differences between the upper and the lower retina, and they will show that a consistent superiority of the upper retinal system can also be found on a perceptual level. This illustrates that the interpretation of the electrophysiological results described in the previous sections is not restricted to a small and highly specialized field of research, but is paralleled by behavioral concomitants. 


\subsection{Temporal Sensitivity ${ }^{4}$}

The temporal resolution capacity of the visual system depends both on physical stimulus variables such as flash duration, intensity, size, and spectral composition of the test light, and on physiological and subject conditions such as state of adaptation or retinal location (for reviews see Landis 1954; Brown 1965; Shickman 1981). Differences between rod and cone functions are reflected in different critical flicker fusion frequency (CFF) values obtained in the center and the periphery of the retina. For more practical clinical applications, the visual field was mapped by Phillips (1933), Hylkema (1942), and Kleberger (1955) using the CFF. These authors described different sensitivities of various parts of the visual field.

The sensitivity to flickering light is not only determined by retinal mechanisms (Dodt 1951; Dodt and Enroth 1954; Grüsser and Kapp 1958; Grüsser and Rabelo 1958); central parts of the visual system also contribute to the CFF, as has been demonstrated by binocular stimulation experiments in humans (Simonson 1958; Lipkin 1962; Zubek and Bross 1972, 1973). This interpretation was supported by Granit (1955), who found that central electrical stimulation modulates the ganglion cell activity of the retina. In addition, Dodt (1964) showed in the rabbit that tetanic stimulation of the optic tract has a direct effect on the retinal CFF. Van de Grind et al. (1973) give a more detailed review of binocular CFF and of cortical neural responses indicating binocular interactions.

Determination of CFF thus yields a global measure of the temporal sensitivity of the visual system, and it is used in this section to investigate functional differences between upper and lower, and between nasal and temporal perifoveal retinal areas. In contrast to Hylkema (1942), who investigated the retinal periphery in order to determine the visual field, in the present experiments the perifoveal regions were examined. In a second experiment, the time thresholds for the more demanding task of double flash discrimination (DFD) were determined.

Fifteen adults between 19 and 32 years of age with normal vision participated in both experiments. Stimuli were presented by the lamp of a GRASS PS2 photostimulator (white light between 450 and $650 \mathrm{~nm}$, exposure $0.16 \mathrm{~lx} / \mathrm{s}$, fused flashes 2.6 candela $/ \mathrm{m}^{2}$ ) which was mounted in the center of a vertical white wooden board with a hole of $2.5^{\circ}$ diameter. Small red LEDs $\left(9.8^{\prime}\right.$ diameter $)$ located at $5^{\circ}$ and $10^{\circ}$ along both directions of the horizontal and vertical meridians served as fixation points. Time thresholds were measured by the method of adjustment at these eccentricities and also with central fixation. This yielded nine measured values for each subject for both CFF and DFD.

After 15 min dark adaptation, the subject fixated monocularly, with his dominant eye, either the center of the display or one of the peripheral fixation points from a distance of $174 \mathrm{~cm}$. In the CFF experiment, the subject controlled the stimulus frequency, and was asked to set the frequency so that he could not quite discriminate single flashes. In the DFD experiment double flashes occurred once per second, and the subject's task was to set the interflash interval so that double

\footnotetext{
${ }^{4}$ The results of this section have been published elsewhere (Skrandies 1985a)
} 
flashes could no longer be perceived. The interflash interval was monitored on an oscilloscope screen with a time resolution between 1 and $4 \mathrm{~ms}$. The CFF values were expressed as flashes per second, while DFD thresholds were measured in milliseconds. After several practice trials, four to six measures were obtained at each of the nine retinal stimulus locations in a quasi-randomized order. The repeatability of the settings was good, and mean CFF or DFD values of each location were used for statistical analysis. Corresponding locations in opposite hemiretinal areas were compared by paired t-tests.

\subsubsection{Results on Critical Flicker Fusion Frequency}

The data of each subject were expressed as percentages of the respective central value. Figure 36 shows the man data of 15 subjects measured in the central $10^{\circ}$ of the retina as isopercentage lines which connect areas of equal temporal sensitivity. The zero isopercentage line runs from the lower nasal part through the center to the upper temporal part, and temporal and lower retinal areas are less sensitive than the nasal and upper areas. This plot rests on interpolated values, and statistical comparisons were performed on the differences between the nasal and temporal and between the upper and the lower hemiretinal CFF values at the retinal locations actually measured. The grand mean CFF value (over all conditions and subjects) was 19.35 flashes per second. In paired t-tests on the individual data, the differences between nasal and temporal CFF were significant only at $5^{\circ}$ eccentricity $(t=1.87, p<0.05)$, while the differences between upper and lower areas were significant at both eccentricities. The mean results (CFF values in $\mathrm{Hz}$ ) and significance levels are summarized in Table 4. In the temporal retina the mean CFF was $92.2 \%$ of the nasal value at $5^{\circ}$ while at $10^{\circ}$ it reached

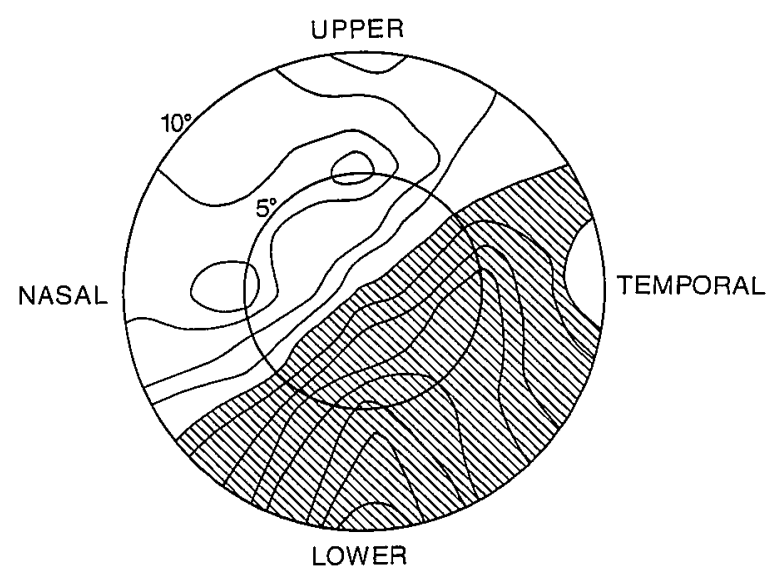

Fig. 36. Retinal distribution of perifoveal mean CFF values (\%) of 15 subjects. The central value is set to $100 \%$, isopercentage lines connect points of equal percentage values. Lines are in steps of $1 \%$. CFF in the shaded areas is smaller, in the white areas larger than the central CFF. The inner circle represents $5^{\circ}$, the outer circle $10^{\circ}$ retinal eccentricity. (From Skrandies 1985a: by permission of Gordon and Breach Science Publishers, London) 
Table 4. Mean CFF values $(\mathrm{Hz})$ of 15 subjects

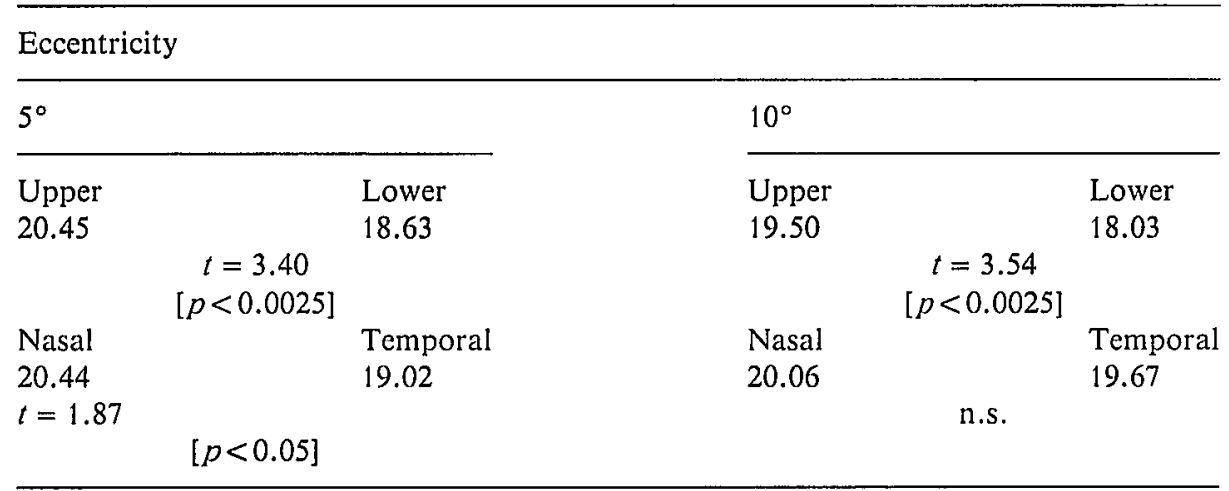

Paired t-tests were used for statistical analysis

$97.8 \%$ of the nasal values. The mean differences between the upper and lower areas were close to $10 \%$ : at $5^{\circ}$ the values for the lower retinal averaged $90.2 \%$ of the upper retinal CFF, and at $10^{\circ}$ the lower retinal CFF values were $91.8 \%$ of the upper retinal CFF values.

\subsubsection{Results on Double Flash Discrimination}

The results obtained for DFD thresholds were similar to the CFF data. Figure 37 shows the sensitivity distribution of the 15 subjects across the perifoveal retinal

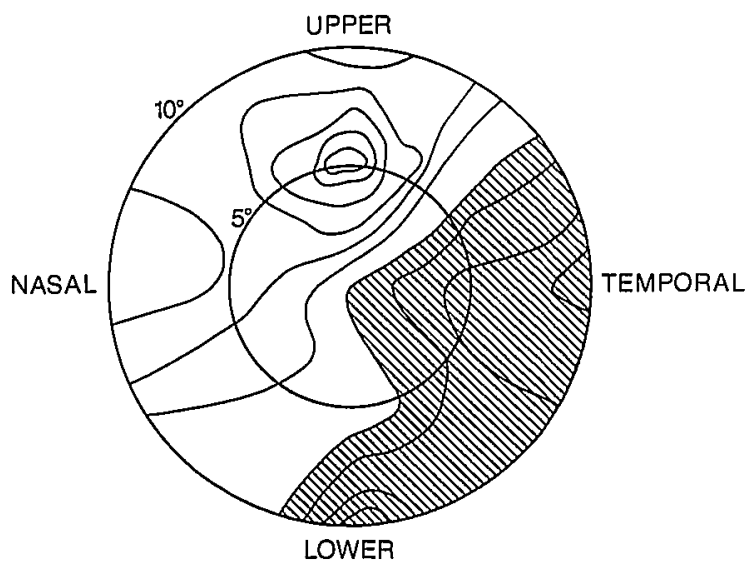

Fig. 37. Retinal distribution of perifoveal mean DFD threshold values (\%) of 15 subjects. The central value is set to $100 \%$, isopercentage lines connect points of equal percentage values. Lines are in steps of $2 \%$. Threshold values in the shaded areas are smaller, in the white areas larger than those of the central area. The inner circle represents $5^{\circ}$, the outer circle $10^{\circ}$ retinal eccentricity. (After from Skrandies 1985a: by permission of Gordon and Breach Science Publishers, London) 
Table 5. Mean DFD values (ms) of 15 subjects

\begin{tabular}{|c|c|c|c|c|}
\hline \multicolumn{5}{|c|}{ Eccentricity } \\
\hline \multicolumn{2}{|l|}{$5^{\circ}$} & \multicolumn{3}{|l|}{$10^{\circ}$} \\
\hline $\begin{array}{l}\text { Upper } \\
82.75\end{array}$ & $\begin{array}{l}\text { Lower } \\
92.80\end{array}$ & $\begin{array}{l}\text { Upper } \\
91.69\end{array}$ & & $\begin{array}{l}\text { Lower } \\
100.91\end{array}$ \\
\hline \multicolumn{2}{|c|}{$\begin{array}{c}t=2.83 \\
{[p<0.01]}\end{array}$} & \multicolumn{3}{|c|}{$\begin{array}{c}t=2.88 \\
{[p<0.01]}\end{array}$} \\
\hline $\begin{array}{l}\text { Nasal } \\
88.96\end{array}$ & $\begin{array}{l}\text { Temporal } \\
97.32\end{array}$ & $\begin{array}{l}\text { Nasal } \\
87.77\end{array}$ & & $\begin{array}{l}\text { Temporal } \\
100.43\end{array}$ \\
\hline \multicolumn{2}{|c|}{$\begin{array}{c}t=2.66 \\
{[p<0.01]}\end{array}$} & \multicolumn{3}{|c|}{$\begin{array}{c}t=3.04 \\
{[p<0.005]}\end{array}$} \\
\hline
\end{tabular}

Paired t-tests were used for statistical analysis

areas. As in the CFF data, the nasal and upper retinal areas have better time resolutions than the temporal and lower retinal areas (Fig. 37). In these data the zero isopercentage line is somewhat different from that in the CFF plot (see Fig. 36), but all differences between corresponding upper and lower locations and between corresponding nasal and temporal locations were statistically significant. The grand mean threshold (over all subjects and over all retinal locations) was $92.88 \mathrm{~ms}$, and the mean DFD values (indicating the interflash intervals in milliseconds) and significance levels obtained with paired t-tests are given in Table 5. The mean differences between the retinal areas examined were in the same order as those of the CFF thresholds. Lower retinal stimulation yielded thresholds of $112 \%$ of the upper retinal values at $5^{\circ}$ and $110 \%$ at $10^{\circ}$, and the temporal areas showed higher values than the nasal retina: At $5^{\circ}$ the mean values were $109 \%$, and at $10^{\circ} 114 \%$ of the nasal values.

\subsubsection{Relation Between Critical Flicker Fusion Frequency and Relative Luminance}

An additional experiment was performed in order to relate the regional differences in temporal sensitivity to relative luminance thresholds. In two subjects CFF measurements were obtained using test lights at eight different luminance levels. The relation between $\mathrm{CFF}$ and $\log$ filter density is shown in Fig. 38. For both subjects the CFF decreased with increasing filter density, and this relationship showed a high correlation (identical value for both subjects: $r=-0.97$ ). Linear regression lines were determined for each subject individually, and although the subjects have different levels of temporal sensitivity, the slopes of the regression lines in Fig. 38 are identical. For both subjects a difference in CFF of 10 flashes per second corresponds to $1.36 \mathrm{log}$ filter units density.

The basic results of this section are in general agreement with the data of Phillips (1933), Hylkema (1942), and Kleberger (1955); upper and nasal retinal areas show 


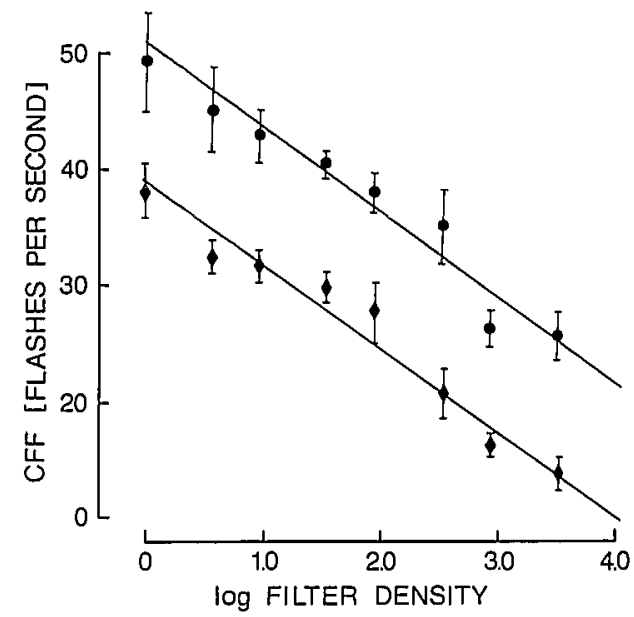

Fig. 38. CFF as a function of $\log$ filter density of two different subjects. (Filled circles or diamonds). Means and standard deviations of ten measurements are illustrated. Light intensity decreases from left to right. The lines are linear regressions computed from individual data. The correlation coefficients and slopes are identical for both subjects $(r=-0.97$, $a=0.136$ ); only the absolute CFF levels are different. (Data from Skrandies 1985a)

higher resolutions than lower and temporal retinal areas (see Sect. 6.2.2.2 for a more complete discussion). However, in the earlier studies the vertical meridian was investigated much further out in the periphery, while in the present experiments significant differences in the temporal resolution capacity were found already in perifoveal retinal areas.

The data presented show a consistent superiority of the upper and nasal over the lower and temporal perifoveal retinal areas. The differences in temporal resolution were found both in the CFF and in the DFD measurements. The discrimination of double flashes yields higher time thresholds than the CFF (Basler 1911; Lindsley and Lansing 1956) because the state of adaptation is different in these two experimental conditions. After prolonged stimulation the subject becomes lightadapted and CFF increases (Granit and Hammond 1931). This was found in the present experiments: for CFF, a mean interflash interval of $51.67 \mathrm{~ms}$ could be resolved, while double flashes could only be discriminated at an interflash interval of $92.88 \mathrm{~ms}$. In addition, all our subjects agreed that the DFD experiment was more difficult to perform than the CFF experiment. Despite this difference in the level of achievement, for the DFD task the relative size of the significant functional differences was of an order similar to the CFF. In both experiments the time resolution values of the lower retinal areas were about $10 \%$ less than those of the upper retina, and the nasal-temporal differences were of a similar magnitude.

The measured values were also expressed as relative luminance thresholds. As expected from the literature (Brown 1965; Shickman 1981), the CFF dropped with decreasing luminance of the test field, and this finding was consistent over subjects. The different CFF levels of the subjects in Fig. 38 reflect individual differences in threshold criterion. Most important, the relationship between CFF and $\log$ filter density showed a high negative correlation, and the slope of the regression lines was identical for both subjects. For the population data, the mean CFF differences between the upper and lower retinal areas correspond to 0.244 log filter density units, which means that the lower retinal areas need about 1.75 times more light in order to achieve the same temporal resolution as the upper 
retinal areas. The nasal-temporal difference averaged 0.123 log filter density units, corresponding to a light intensity factor of 1.33 .

These small differences are very consistent in the subject population, and the magnitude of the effect of the order of $10 \%$ still permits an uniform functioning within the perifoveal visual field. In summary, statistically significant differences exist for both the CFF and the DFD task, showing a higher temporal sensitivity of the nasal and upper than of the temporal and lower retinal areas.

\subsection{Visual Acuity and Contrast Sensitivity ${ }^{5}$}

There are reports suggesting regional differences in visual acuity between upper and lower retinal areas (Landolt and Hummelsheim 1904; Low 1943). Using Landolt rings of high contrast, Millodot and Lamont (1974) determined the visual acuity along the vertical meridian in three subjects. This section will report acuity differences between upper and lower perifoveal areas using a more complete assessment of visual resolution.

The measurement of human contrast sensitivity introduced by Schade (1956) and applied to the visibility of complex visual grating patterns by Campbell and Robson (1968) allows a more detailed examination of spatial visual resolution than conventional measures of visual acuity, which emphasize primarily high spatial frequency resolution at high contrast levels. Clinical studies on amblyopic patients (Gstalder and Green 1971) as well as studies on patients with multiple sclerosis (Regan et al. 1977) or with cortical lesions (Bodis-Wollner and Diamond 1976) demonstrated that normal visual acuity does not rule out perceptual disturbances. Brettel et al. (1982) and Lawden (1982) found that in amblyopic patients, not only contrast sensitivity but also sensitivity to spatial phase may be disrupted. In addition, Skrandies and Gottlob (1986) showed that patients suffering from Parkinson's disease also had normal conventional visual acuities but significantly reduced contrast sensitivity. The measurement of contrast sensitivity can thus be used to study functional characteristics of various retinal areas related to visual resolution over the whole range of spatial frequencies from very coarse to very fine visual targets which are believed to be analyzed by the visual system in independent spatial frequency channels (for review see Braddick et al. 1978).

In this section the contrast sensitivity thresholds of upper and lower perifoveal hemiretinal regions will be compared over a range of different spatial frequencies. In addition, we will show that these data also suggest differences in visual acuity. Twenty subjects between 19 and 32 years of age with normal or corrected-to-normal vision were presented static sine-wave-modulated vertical grating patterns of a mean luminance of 1.21 candela $/ \mathrm{m}^{2}$ on the screen of a R5030 Tektronix oscilloscope (P31 phosphor; field size $5.99^{\circ} \times 7.40^{\circ}$ arc). Gratings of seven different spatial frequencies were used: $0.45,0.62,1.24,1.96,4.58,9.13$, and 13.74 cycles per degree $(\mathrm{c} / \mathrm{deg})$. From a distance of $100 \mathrm{~cm}$, the subject fixated monocularly, with his dominant eye, either the center of the display or one of the peripheral fixation points $5^{\circ}$ above or below the edge of the display. An ascending method of limits was used. After several practice trials, five measurements were

${ }^{5}$ Results of this section have been published elsewhere (Skrandies 1985b) 
obtained at each of the seven spatial frequencies. The repeatability of the settings was good as quantified by the very low standard deviations.

For each subject and each retinal location the contrast sensitivity function was determined, and the lowest value of each curve defined maximal sensitivity. An analysis of variance established a significant effect of retinal stimulus location, and the shapes of the contrast sensitivity functions were also compared statistically using paired t-tests. The significance levels of the t-values were corrected for multiple comparisons according to Holm's (1979) procedure, and all results presented were significant at an alpha level of $p<0.05$ unless stated otherwise. The values obtained at the three highest spatial frequencies were used to compute linear regressions between contrast threshold and spatial frequency for each retinal location separately. By extrapolating the regression lines to the high-frequency cutoff point (i.e., to $100 \%$ contrast) conventional visual acuity was estimated.

The grand mean contrast sensitivity functions computed from the data of $20 \mathrm{sub}$ jects obtained at the three different retinal locations are shown in Fig. 39A. The overall contrast levels are different, with central stimuli showing the lowest threshold values (mean over all spatial frequencies and subjects: $1.46 \%$ contrast), followed by upper retinal stimuli (mean over all spatial frequencies and subjects: $1.93 \%$ contrast). The lower hemiretina shows the highest contrast threshold values at all spatial frequencies (mean over all spatial frequencies and subjects: $2.42 \%$ contrast). The shapes of the contrast sensitivity curves were significantly different, as revealed by an analysis of variance (paired t-tests comparing retinal locations using all the data irrespective of spatial frequency also showed significant differences between the three retinal locations).
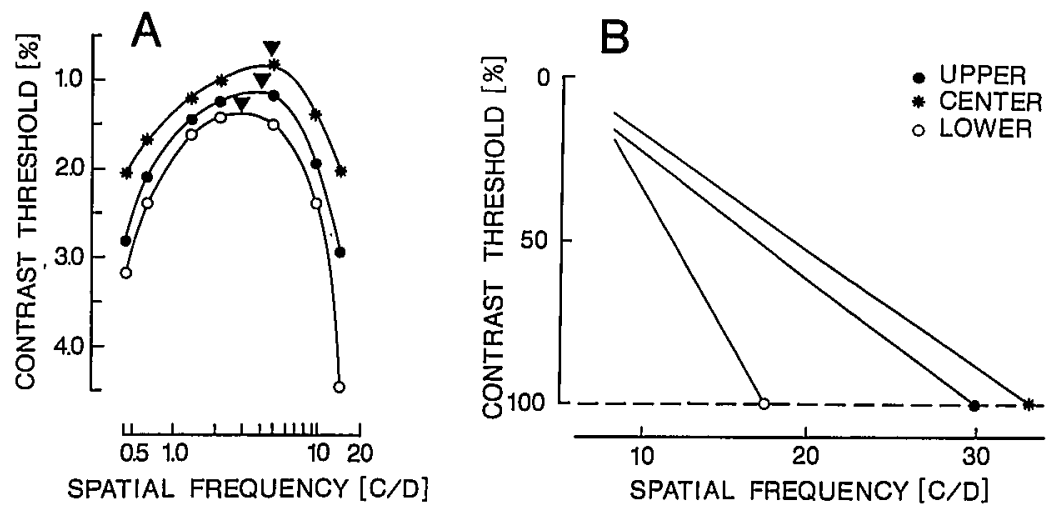

Fig. 39. A Mean contrast sensitivity curves of 20 subjects for central and for upper and lower hemiretinal perifoveal areas. Arrowheads indicate the mean spatial frequencies which yielded the highest individual contrast sensitivities. The differences in overall contrast level, as well as in the spatial frequencies to which subjects were most sensitive, were significantly different. (After Skrandies 1985b) B Linear regressions between contrast threshold and spatial frequency for upper, central, and lower retinal stimuli. Extrapolation of the regression lines to the high-frequency cutoff point (i.e., to $100 \%$ contrast) gives an estimate of conventional visual acuity. Cutoff frequencies at $100 \%$ contrast were $33.16 \mathrm{c} / \mathrm{deg}$ for central, $29.98 \mathrm{c} / \mathrm{deg}$ for upper, and $17.50 \mathrm{c} / \mathrm{deg}$ for lower retinal stimuli (see text) 
The lowest contrast thresholds occur at different spatial frequencies for the different retinal locations, indicating sensitivity differences (Fig. 39A). The spatial frequencies at which each subject was most sensitive were also compared between retinal locations, and all paired t-tests on spatial frequency differences between central (mean $4.31 \mathrm{c} / \mathrm{deg}$ ), upper (mean $3.85 \mathrm{c} / \mathrm{deg}$ ), or lower (mean $2.63 \mathrm{c} / \mathrm{deg}$ ) retinal stimulus locations were significant, as summarized in Fig. 40.

In addition, comparisons of the contrast values at each of the seven measured spatial frequencies were made: All statistical comparisons were highly significant, indicating that there are regional retinal differences not only in visual acuity, as suggested by the different contrast levels at the highest spatial frequency, but that the global differences are not restricted to individual spatial frequency channels. For the three highest spatial frequencies the correlations between contrast threshold and spatial frequency were highly significant $(p<0.005)$ at all retinal locations investigated, ranging from 0.6 to 0.7 . The linear regressions between contrast threshold and spatial frequency are illustrated in Fig. 39B. Central and upper stimuli yielded similar slopes of the lines, and comparable cutoff frequencies at $100 \%$ contrast ( $33.16 \mathrm{c} / \mathrm{deg}$ for central, $29.98 \mathrm{c} / \mathrm{deg}$ for upper retinal stimuli). The cutoff point determined for lower retinal stimuli was $17.50 \mathrm{c} / \mathrm{deg}$. When expressing these cutoff frequencies as visual acuity, the grand mean data of Fig. 39B yield acuity values of 1.1 for central, 0.99 for upper, and 0.58 for lower retinal stimuli. Foveal stimuli consistently yielded the highest contrast sensitivities and the highest cutoff spatial frequencies (i. e., visual acuites), as well as a shift of the maximal sensitivity toward higher spatial frequencies than perifoveal stimuli. This reflects the known anatomical and functional superiority of the fovea with its dense packing of cones and its low amount of convergence onto retinal ganglion cells, enabling high spatial resolution.

The findings on upper/lower hemiretinal differences are in agreement with those of Landolt and Hummelsheim (1904), Low (1943), and Millodot and Lamont (1974), who determined the conventional visual acuity at high contrast levels. Millodot and Lamont (1974) described differences in visual acuity only beyond $5^{\circ}$ eccentricity, and Low's (1943) data were obtained at $30^{\circ}$ eccentricity. Wertheim (1894) had also found higher visual acuities for the upper hemiretina only beyond $20^{\circ}$ eccentricity. In contrast to these authors, the present data show that differences between the upper and the lower hemiretinal areas exist in the perifovea at as little as $5^{\circ}$ eccentricity (see Sect. 6.2.2.3 for a more complete discussion of the earlier literature).

The contrast sensitivity functions of the perifoveal retinal regions presented in this chapter show a significant superiority of the upper over the lower retinal areas.

Fig. 40. Mean maximal contrast sensitivities obtained from 20 human subjects. Statistically significant differences between central, upper, and lower retinal perifoveal area were established by paired t-tests

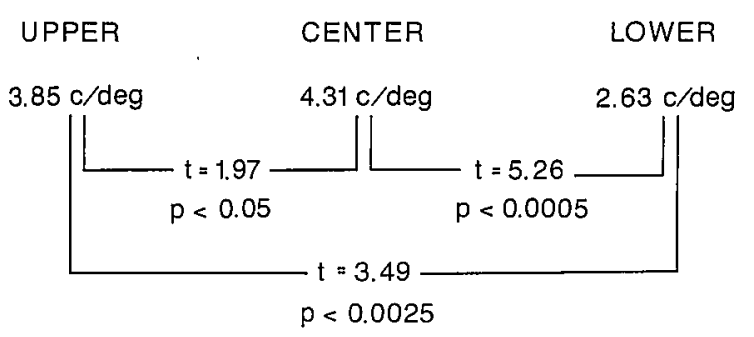


These differences in contrast sensitivity were not restricted to high spatial frequencies or estimated conventional visual acuities, but were observed at all spatial frequencies. Differences in maximal sensitivity, as well as the different shapes of the contrast sensitivity functions, suggest a global functional superiority of the upper over the lower perifoveal hemiretinal areas. This functional difference is not restricted to certain individual spatial frequency channels.

\subsection{Discrimination of Meaning}

Pattern discrimination of meaningful stimuli can be considered a realistic visual task in everyday life. In this section consequences in behavioral performance which arise from the physiological differences between the upper and the lower retina are illustrated. In a simple pattern discrimination task, digits were presented at an eccentricity of $5^{\circ}$ either above or below the fovea, and the subject had to report which digit he had seen. In contrast to experiments using checkerboard or grating stimuli, the discrimination of numbers presented on a computer terminal is not an artificial, laboratory-bound experimental task for the subject. Fifteen young adults (19-33 years of age) with normal vision participated in the experiments. One of ten different digits $(0-9)$ was flashed in a randomized order on the terminal of an Apple Ile computer, $5^{\circ}$ above or below a central fixation point consisting of a light cursor. The size of the test target subtended a $30^{\prime}$ arc. Both sequences of stimulus location and digits were randomized. The subjects responded by entering the appropriate number on the keyboard. After a programmed delay of $1 \mathrm{~s}$, the next stimulus appeared. Thus each subject was free to work at his own pace. Each subject performed 600 trials. Accuracy in performance was stressed, and a forced-choice task was employed. For analysis, percentages of correct responses were computed and the discrimination performance of upper and lower retinal areas was compared using Wilcoxon tests. Luminance and contrast of the display were adjusted for each subject individually to yield a mean above $50 \%$ correct responses.

The performance of all subjects was significantly above the chance level of $10 \%$. The mean of correct responses computed over all subjects and all retinal locations was $74.39 \%$ correct responses. There were no obvious differences in discrimination performance between individual digits. Because a forced-choice task was employed, confusions mainly arose between similarly shaped digits (e. g., 1,4,7 or $6,8,9,0$ were incorrectly reported).

The individual data of the 15 subjects and the group means are shown in Fig. 41. In 14 of the 15 subjects stimuli presented to the upper retina yielded better discrimination performances than those presented to the correponding lower retinal area. With upper retinal stimuli $78.63 \%$ correct discriminations were obtained, whereas only $70.14 \%$ of the lower retinal stimuli were correctly discriminated. This difference was statistically significant in the subject population (Wilcoxon $T=14, n=15, p<0.005$ ).

In this experiment, fixation was deliberately not very strictly controlled. Nevertheless, systematic significant differences in discrimination performance were found. In order to achieve a high level of accuracy, the subjects' best strategy was to fixate at the center of the screen, because the location of the expected stimulus 


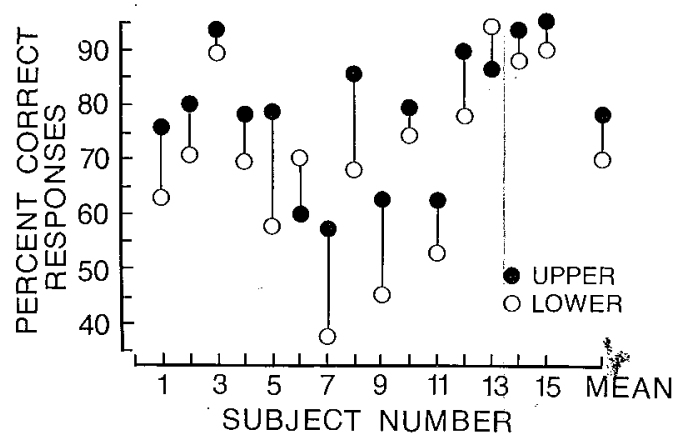

Fig. 41. Percentages of correct responses in a pattern discrimination task. Individual data of 15 subjects as well as the means are shown. Stimuli presented to the upper retina yielded significantly better discrimination performances than those presented to the corresponding lower retinal areas (78.63\% vs $70.14 \%$ correct discriminations)

could not be predicted. The subjects were naive as to the exact purpose of the experiment and had been encouraged to work as accurately as possible at their own pace. As expected from the data on temporal resolution and visual acuity presented in Sects. 5.1 and 5.2, stimuli occurring below the fixation point could be discriminated significantly better than those above the fixation point. In this task, too, the stimuli were presented only at $5^{\circ}$ eccentricity, and the upper retinal superiority was significant in the subject population. In addition, we note that the differences between upper and lower retinal performance were again in the order of $10 \%$.

Related differences in detection performance between visual targets presented above or below the fixation point were found in a study on attention and visual search using a much more complex "contingent-display technique". Those results were interpreted as an interaction between attention and sensitivity across the visual field (Prinz 1984; see also Sect. 6.2.2.4). Our data illustrate how, in a simple experimental setup, functional differences between upper and lower retinal areas may be demonstrated. The results were obtained in a common visual task and in a realistic instrumental suround, and they might be of interest in the investigation of man-machine interactions in everyday working conditions.

\section{Evidence from the Literature}

The aim of this section is to provide further evidence of the upper/lower distinction within the visual system by giving background information on what is known from the scientific literature on this topic. It cannot be an exhaustive review, mainly because the differences between the upper and lower visual systems have rarely been investigated directly. Most of the data presented is found in papers concerned with other research aims. It is important to note that this review will not only illustrate that the upper retina is superior over the lower retina (whatever this means in functional, perceptual terms), but also give interesting examples and experimental data which suggest that to a certain degree the upper and lower parts of the retina and the visual system as a whole may be considered independent systems. On the other hand, I do not mean to challenge the integrative action of the visual system.

In order to organize the review, a somewhat arbitrary classification of the literature had to be made, and structural (i. e., anatomical) and functional local 
differences within the visual system will be discussed separately. The first part (Sect. 6.1) is organized according to the anatomy of the visual system, describing regional differences at the retina, at midbrain structures, and at the cortex of animals and humans. Functional differences will be described in the second part (Sect. 6.2), which reviews findings in animals and humans, while in the last part (Sect. 6.3) clinical observations will further illustrate that the upper and lower visual systems may behave as independent units.

\subsection{Anatomical Findings}

\subsubsection{Animal Data}

\subsubsection{Retina, Optic Nerve, and Optic Tract}

There is some anatomical evidence of differences between upper and lower areas in the eye. Dogs and cats have a tapetum beneath a portion of their retina, and the pigment epithelium cells overlying the tapetum are not pigmented. The tapetum presumably improves scotopic vision by reflecting the incoming light, and it has been reported that the tapetum lucidum is restricted to the upper retinal half of the cat eye (Bishop et al. 1962; see also Fig. 1 of Niemeyer 1975).

In some fish and amphibians one finds very elaborate adaptations of the visual system to the environment of the animal. The four-eyed fish (Anableps anableps) is capable of both aquatic and aerial vision. Its eyes each have two pupils, one oriented toward the upper and one toward the lower visual field, and contain two major visual axes (see Fig. 4.5 of Ali and Klyne 1985). In addition, the retina is divided into a dorsal (upper) and a ventral (lower) part which contain different photopigments. In the ventral part of the retina, which subserves aerial vision, rhodopsin is predominant, while porphyropsin is found mainly in the dorsal, aquatic retina. Rhodopsin and porphyropsin have different spectral absorption spectra (502 nm and $522 \mathrm{~nm}$, respectively), and the upper retina has an increased relative sensitivity toward longer wavelengths. This may be important for aquatic vision, since turbid water scatters shorter wavelengths more strongly than longer wavelengths (Luria and Kinney 1970).

A similar difference in the distribution of retinal photopigments is found in the bullfrog. In the larval stage it inhabits an aquatic environment, and its retina contains mainly porphyropsin. In the adult bullfrog the dorsal retina contains mainly porphyropsin, while rhodopsin predominates in the ventral retina (see Figs. 4 and 5 of Reuter et al. 1971). This might well relate to the fact that the dorsal retina more often looks down into the water whereas the ventral retina more often subserves aerial vision.

The regional distribution of the photoreceptors of the eye has been analyzed by Landau and Dawson (1970). They published some material on the retina of the sea lion, where more photoreceptors were encountered in the upper than in the lower half (see Fig. 3 of Landau and Dawson 1970).

The visual field of most birds is largely monocular, only a very small area being binocular. The binocular field is projected onto a region in the upper temporal quadrant of the retina, the so-called red area. In this region of the retina the inner limbs of the cones contain red oil globules, as found in ducks, chickens, and pigeons (King-Smith 1969). On the basis of this different distribution of red oil 
droplets, the wavelength discrimination capability in the lower visual field is expected to be shifted toward longer wavelengths compared to the discrimination capability in the upper visual field (see Sect. 6.2.1). The red area has a large magnification factor in the tectum which constitutes a major central visual area in the bird. In addition, in this area there is a high density of retinal ganglion cells, suggesting a greater importance of the upper temporal quadrant of the bird retina.

The cell density of the ganglion cell layer of the chick retina was studied by Ehrlich (1981). The highest value, 3000 cells $/ \mathrm{mm}^{2}$, was observed in the center, and cell density dropped sharply in all directions from the central area except for a strip in the superior temporal retina (see Fig. 9 of Ehrlich 1981). Thus, two areas with enhanced visual resolution corresponding to regions of high ganglion cell density exist in the lateral and inferior frontal visual field.

In a quantitative analysis of cat retinal ganglion cell topography, Hughes (1975) showed that ganglion cell density is higher in upper than in corresponding lower retinal areas (see Figs. 7 and 9 of Hughes 1975). In addition, differences in retinal surface area exist along both the horizontal and vertical meridians, with the upper and nasal retina occupying a larger percentage of the total retinal area than the lower and temporal areas. The upper retina represents $60 \%$ and the lower retina $40 \%$ of the total retinal area, while the nasal retina represents $72 \%$ and the temporal retina only $28 \%$ of the total retinal area (see Table 5 of Hughes 1975). According to these data the lower retina of the cat contains 98700 ganglion cells and the upper 118500 , corresponding to $45.4 \%$ and $54.6 \%$ of the total ganglion cell population respectively.

Vaney and Hughes (1976) published data of a cell count in the rabbit retina and reported differences in ganglion cell density in the peripheral retina. For areas above the visual streak 550 cells $/ \mathrm{mm}^{2}$ were reported, while areas below the visual streak contained only 350 cells $/ \mathrm{mm}^{2}$.

Hughes and Wässle (1976) counted the nerve fibers and determined their diameter spectrum in the cat optic nerve. In a section about $2-3 \mathrm{~mm}$ behind the globe they found temporally a region with a high mean density of about 150000 fibers $/ \mathrm{mm}^{2}$. This region was assumed to correspond to the area centralis of the retina, and it extended further into the upper part of the optic nerve. A more homogeneous distribution was observed some $7 \mathrm{~mm}$ behind the globe; where the densities in the upper half were higher than those in the lower half of the optic nerve. Although the retinotopic order in the optic nerve is rather crude, these data agree well with the findings on different ganglion cell densities in the upper and lower retina presented above.

The topography of amacrine cells in the cat retina also shows differences in the distribution along the vertical meridian. Upper retinal areas display a higher cell density than corresponding lower retinal areas, and the density gradients appear to decrease continually from the center toward the periphery in the lower retina while in the upper retina a plateau of equal cell density is observed (see Fig. 2 of Schmidt et al. 1985; Vaney 1985). On the other hand, Oyster et al. (1985) reported that the distribution of dopaminergic displaced amacrine cells as well as that of dopaminergic interplexiform cells showed highest cell densities in the inferior part of the cat retina (see Fig. 2 of Oyster et al. 1985).

The topography of horizontal cells of the cat retina was examined by Wässle et al. (1978). Cell density was reported to be highest in the center and to decrease 
toward the periphery. Inspection of the isodensity lines reveals an asymmetrical distribution of horizontal cells in the upper and lower retina, with the upper retina showing higher cell densities (see Fig. 9 of Wässle et al. 1978). The dendritic fields of the horizontal cells were found to increase from the center toward the periphery of the retina, and the dendritic field diameters appear to be slightly smaller in the upper than the lower retina, suggesting a smaller amount of convergence of photoreceptors onto retinal ganglion cells (see Fig. 10 of Wässle et al. 1978).

There is a rough retinotopic arrangement of the nerve fibers forming the cat optic tract with a representation of the lower visual field in its dorsolateral part (Torrealba et al. 1982). In an electrophysiological study Aebersold et al. (1981) investigated the representation of the visual field in the optic chiasm and the optic tract of the cat. They recorded the electrical activity of 749 fibers from 19 adult cats. Fibers originating in the upper hemiretina were much more frequently encountered than fibers from the lower retina. This ratio averaged $63 \%: 37 \%$ and was similar in the optic chiasm and the optic tract. From these data the authors concluded that the upper hemiretina is more strongly represented in the visual system and that it presumably possesses a higher retinal ganglion cell density than the lower hemiretina.

\subsubsection{Midbrain Structures}

In various species, differences between upper and lower areas of the visual field have been demonstrated even at the level of the midbrain.

The pigeon retina receives centrifugal nerve fibers terminating on amacrine and displaced ganglion cells in the retina. These fibers originate in the isthmo-optic nucleus, where Holden and Powell (1972) described a greater representation of the lower visual field and particularly of the red area of the retina. The visual field is mapped retinotopically on the optic tectum, with larger areas occupied by the fovea and the red area (Clarke and Whitteridge 1976). The wulst of the pigeon, reminiscent of the mammalian visual cortex, is retinotopically organized and has a columnar arrangement. Most visually active units in various layers of the wulst were found to have their receptive field centers in areas corresponding to the fovea and the red area of the retina (Gusel'nikow et al. 1976, cited by Emmerton 1983a). The overlapping binocular visual field of the pigeon is located in the inferior frontal field and corresponds to the red area (for review see Emmerton 1983b).

According to Whitteridge (1973) the horizontal meridian divides the surface of lamina $A^{\prime}$ of the cat lateral geniculate nucleus (LGN) unequally. The lower visual field is projected to an area which is about twice as big as that devoted to the upper visual field. In particular, the visual field just below the horizontal meridian and up to an eccentricity of about $10^{\circ}-20^{\circ}$ is extensively represented. Such differences between the upper and lower visual fields in the LGN of the cat, with the lower visual field occupying a larger area than the upper visual field, had been reported earlier by Whitteridge and Seneviratne (1962) and by Seneviratne (1963). In small animals like the rat, mouse and frog, some evidence has been obtained that an asymmetry exists in midbrain structures of the visual system, favoring the upper part of the visual field. 
Nagata and Hayashi (1984) investigated the projection of the visual field onto the ventral lateral geniculate nucleus (LGNv) of the rat. This structure shows a retinotopic organization where the nasal visual field is represented dorsally and the temporal visual field ventrally. The upper visual field projects to rostrolateral and the lower visual field to caudomedial parts of the LGNv. Nagata and Hayashi (1984) recorded electrical activity from 194 cells of the rat LGNv, and analyzed the distribution of the corresponding receptive field centers in the visual field. They found a more extensive representation of the upper visual field (up to $40^{\circ}$ eccentricity) than of the lower visual field (up to $20^{\circ}$ eccentricity), but there was a higher cell density representing the lower visual field. This difference in cell density was most evident when comparing receptive field centers in an area up to $20^{\circ}$ above the horizontal meridian with those in the corresponding area extending to $20^{\circ}$ below the horizontal meridian (see Figs. 3 and 4 of Nagata and Hayashi 1984).

A similar asymmetry between upper and lower visual fields exists in the mouse visual system. The visual projections to the mouse superior colliculus were mapped with microelectrode recordings by Dräger and Hubel (1976) in seven animals. It was found that the upper visual field is disproportionally represented (see Fig. 2 of Dräger and Hubel 1976). An area of high magnification was reported some $30^{\circ}$ above the horizontal meridian, indicating that a larger amount of tectal surface is devoted to a point in the upper than in the lower visual field.

The nucleus isthmi of the frog visual system shows an orderly topographical organization, as has been demonstrated using neuroanatomical techniques (Scalia 1976), and an interesting functional difference between the upper and lower visual field projections to the frog nucleus isthmi was reported by Shurong et al. (1981). They recorded visual responses of 125 neurons and confirmed the retinotopic representation of the visual field. Only a small number of binocular neurons were found, mainly located in the medullary part of the rostral half of the nucleus (Fig. 4 of Shurong et al. 1981). This part of the nucleus isthmi receives projections from lower retinal areas, and it appears conceivable that this finding is a physiological correlate of the greater importance of the frog binocular upper visual field.

\subsubsection{Cortical Areas}

An orderly retinotopic mapping was reported for most cortical visual areas in all species studied. There are several cortical areas involved in visual information processing, and among the best studied are the primary visual cortex (area 17 of Brodmann) and the circumstriate belt (areas 18 and 19). Other visual cortical areas exist and have been investigated, and the functional topography of the visual cortex has been summarized by Creutzfeldt (1983) and van Essen (1985). In this section, mainly differences in the cortical representation of the upper and lower visual fields will be reviewed. The reported systematic differences in the amount of cortex devoted to the upper or lower hemiretinal projections, as well as the differences in neuronal response properties in extrastriate cortical areas representing the upper or lower visual field, yield a rather consistent picture.

Bilge et al. (1967) mapped the visual cortex of the cat by recording evoked potentials and single unit activity. One of their main findings was that the cortical sur- 
face area devoted to the upper visual field (i. e., the lower hemiretina) was considerably smaller than that devoted to the lower visual field. This was true for both area 17 (visual I) and area 18 (visual II).

In area 17 of the cat, extensive microelectrode mapping experiments were performed by Tusa et al. (1978). They found that a larger cortical area is devoted to the lower than to the upper quadrant of the visual field (see Fig. 6 of Tusa et al. 1978).

Van Essen et al. (1984) studied the visual field representation in the striate cortex of six macaque monkeys, presenting microelectrode data from a total of 156 recording sites. From their summary of all data obtained it is clear that the upper hemiretina is distributed over a larger cortical area than the lower hemiretina (see Fig. 4 of von Essen et al. 1984). This difference was most pronounced for the visual fields within $45^{\circ}$ above or below the horizontal meridian.

Standage and Benevento (1983) demonstrated a topographical projection onto the visual midtemporal area (MT), which is located in the superior temporal sulcus of the macaque monkey, and van Essen et al. (1981) found that upper and lower visual fields are represented asymmetrically in this area. Only a total of $26 \%$ of the receptive fields analyzed had their centers above the horizontal meridian, and there was also underrepresentation of receptive field centers close to the upper vertical meridian.

Some of the intrahemispheric projections in the macaque monkey visual cortex are different for the upper and lower visual fields. The dorsal part of the striate cortex (V1), which is connected to the upper hemiretina, projects to visual area V3, while the ventral part of V1 representing the lower retina shows no corresponding projection (van Essen et al. 1986).

In addition, there are differences in physiological response properties of neurons in extrastriate cortical areas representing the upper or lower quadrants of the visual field. In the macaque visual cortex the area V3 located in the dorsal extrastriate cortex contains a representation of the lower visual field, while the ventral posterior area (VP) is located in the ventral extrastriate cortex and represents the upper visual field. Burkhalter et al. (1986) reported that in V3 a larger percentage of neurons were direction-selective, while in VP more neurons were colorselective. This indicates that there are functional asymmetries relating to the processing of information about motion and color in extrastriate cortical areas which represent the upper or lower visual field.

A double representation of the lower visual quadrant was reported to exist in the prelunate gyrus of the rhesus monkey (V4 area). Baizer and Maguire (1983) analyzed single unit activity obtained at 358 recording sites in five hemispheres of three monkeys. Their main finding was that most of the V4 complex consisted of two visual areas in the posteromedial and the anterolateral parts. These areas were retinotopically organized, and both represented the contralateral, lower visual quandrant. The receptive fields found were very large, and Baizer and Maguire (1983) concluded that the neurons in the V4 area do not contribute to the spatial visual resolution; rather, these neurons are concerned with more "global" aspects of vision.

There are also efferent connections from the visual cortex to subcortical areas, and the corticoponto-cerebellar pathways are believed to be involved in the guidance of movement (Glickstein et al. 1972). In cats, the visual areas 17, 18 and 
19 have been shown to project to rostral pontine nuclei which in turn project to the cerebellum. The corticopontine neurons in area 18 are binocularly driven, have large receptive fields, and they are directionally sensitive and respond to downward movement at high velocities. Cells in the rostral pons show similar behavior and their large receptive fields respond to downward movements (Gibson et al. 1978). This organization enables the coding of movement of the terrain and gives the animal information about its locomotion. For such functional coordination of the visual input with motor performance, the lower parts of the visual field are most important. This is reflected in the results of anatomical studies on the corticopontine connections: In the cat visual cortex it has been demonstrated that in all visual cortical areas studied, the cortex representing the lower visual hemifield has a significantly higher number of corticopontine cells than the cortex representing the upper hemifield (Bjaalie and Brodal 1983; Bjaalie 1985). The corticopontine cells of the visual cortex are presumably involved in the transmission of spatially relevant information, and Bjaalie and Brodal (1983) showed in four cats that a mean of $80.5 \%$ of all cells labeled with horseradish peroxidase were found in parts of area 17 which represented the lower visual field. In a recent study on the distribution of corticopontine cells in areas 18 and 19 of the cat, Bjaalie (1985) found a similar overrepresentation of the lower visual field in these cortical areas.

\subsubsection{Human Data}

\subsubsection{Retina}

Most of the data on the anatomy of the mammalian retina have been obtained from the cat, and there have been few studies on the human retina. Østerberg (1935) gave a quantitative account of the distribution of rods and cones in the retina of a 16-year-old boy. From this quantitative study Østerberg (1935) concluded that there are higher densities of both classes of photoreceptors in the upper and nasal than in the lower and temporal parts of the retina (Figs. 6 and 7 of Østerberg 1935).

A recent report on retinal punches obtained at $20^{\circ}$ and $40^{\circ}$ eccentricity along the vertical and horizontal meridians of the human retina confirms a higher density of photoreceptors in the nasal and upper than in the temporal and lower retinal areas (Curcio et al. 1985).

Van Buren (1963) published data on the distribution of retinal ganglion cells along the major meridians. His data obtained from six human retinae are summarized in Fig. 42, which shows the distribution of ganglion cells along the vertical meridian. Two striking differences between the upper and lower retinal areas may be seen: First, the decay of ganglion cell densities is steeper in the lower retina. From about $10^{\circ}$ eccentricity on, the lower retina displays a smaller ganglion cell density. The overall number of ganglion cells averaged 2325058 for the upper meridian while along the lower retinal meridian 2137840 ganglion cells were counted (van Buren 1963, p. 64).

Second, there is a difference in the amount of overlapping ganglion cell layers (Fig. 42). More centrally, up to five layers are found, but toward the periphery the 


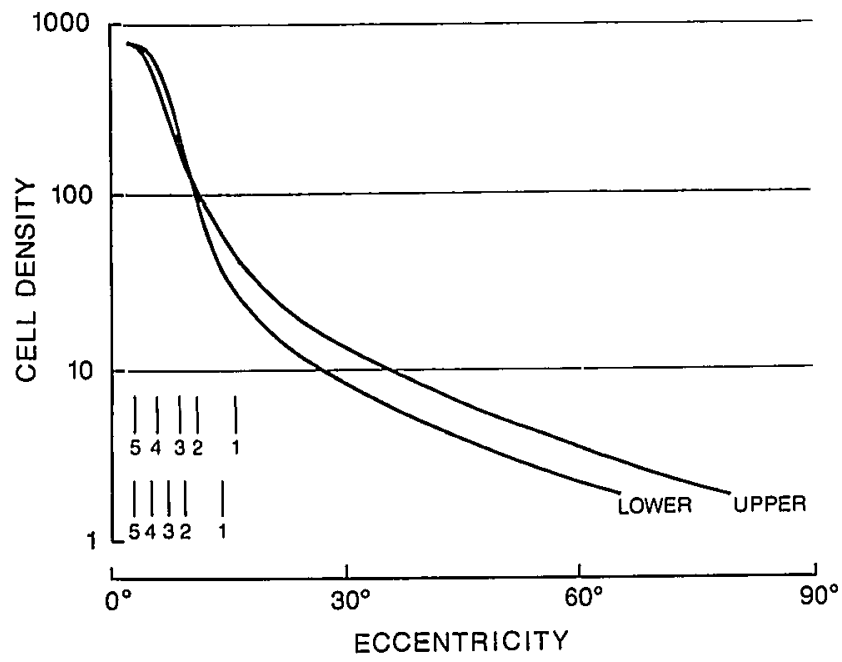

Fig. 42. Ganglion cell distribution in the human retina along the vertical meridian. Cell densities in cells per square micrometer. Numbers indicate the margins of cell layers of various thickness. (Measured from Fig. 36 of van Buren 1963)

Table 6. Margins of retinal ganglion cell layers of various thickness in the human retina giving retinal eccentricities in degrees of visual angle. (Data measured from Fig. 36 of van Buren 1963)

\begin{tabular}{lcc}
\hline Number of cell layers & Upper retina & Lower retina \\
\hline 1 & $15.66^{\circ}$ & $14.50^{\circ}$ \\
2 & $11.00^{\circ}$ & $9.66^{\circ}$ \\
3 & $9.00^{\circ}$ & $7.50^{\circ}$ \\
4 & $6.33^{\circ}$ & $5.00^{\circ}$ \\
5 & $3.33^{\circ}$ & $2.66^{\circ}$ \\
\hline
\end{tabular}

ber of layers is reduced to one (see also Hebel and Holländer 1983). Analysis of Fig. 42 indicates that in the upper retinal areas the overlapping of ganglion cell layers extends much further out toward the periphery than in lower retinal areas. The eccentricities of the margins between the five ganglion cell layers were measured from van Buren's (1963) original data, and the results are presented in Table 6. All layers show a larger extension toward upper retinal areas, indicating a higher ganglion cell density in the upper than in the corresponding lower hemiretinal areas.

A related set of data on the distribution of ganglion cells across the human retina has been presented by Stone and Johnston (1981). They measured the ganglion cell density in one human retina, and displayed their results in the form of equidensity maps. The ganglion cell density along the vertical meridian was measured from their distribution map (Fig. 8 of Stone and Johnston 1981), and is illustrated in Fig. 43. Similar to van Buren's (1963) data, higher cell densities are 
Fig. 43. Ganglion cell density along the vertical meridian of one human retina. Note consistently higher ganglion cell densities in the upper retina. (Data measured from Fig. 8 of Stone and Johnston 1981)

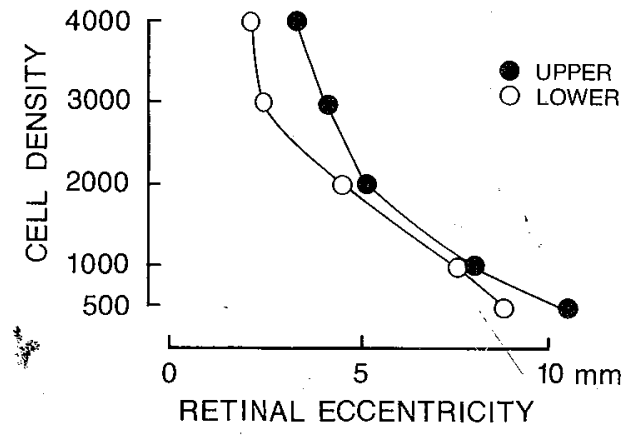

found in the upper retinal areas. This finding was not restricted to the areas along the vertical meridian; higher cell densities for the upper retina were also found in more nasal and temporal retinal areas (see Fig. 8 of Stone and Johnston 1981).

\subsubsection{Cortical Areas}

As in other higher mammals, the major part of the primary visual cortex of man is located on the medial aspects of the occipital cerebral hemispheres. Most of this primary visual area is folded into the calcarine fissure, and the fibers of the optic radiation representing the upper part of the visual field (i. e., the fibers originating in the lower retinal area) terminate below the calcarine fissure, while the lower visual field (i. e., the upper retina) is represented in the upper lip of the calcarine fissure.

Much of what is known about the cortical projections in man has been established in patients with localized lesions of the visual cortex (e. g., Holmes 1945; Spalding 1952; Teuber et al. 1960). The topographical mapping of the visual field onto the visual cortex is weighted by the functional importance of the retinal areas, which results in a more extensive representation of the foveal region. This has become known as cortical magnification (Whitteridge 1973).

Brindley and Lewin (1968) showed that electrical stimulation of an array of electrodes implanted over the calcarine cortex in a blind patient resulted in visual sensations whose topographies were consistent with the established maps of the visual cortex.

Newman et al. (1984) described two patients with altitudinal hemianopia caused by occipital infarctions. Computer tomograms confirmed a direct correlation of the visual field defect with the site of infarction: Defects in the superior visual field were associated with lesions in the lower bank of the calcarine fissure, while a patient with a lower visual field defect showed infarctions in the upper banks of the calcarine fissure.

Recent technological advances have made it possible to map cortical areas of the human brain also in terms of their functional activity. Phelps et al. (1981), Greenberg et al. (1981), and Schwartz et al. (1984) described the metabolic mapping of functional activity of the human brain following visual stimulation, and their data are in line with the cortical projections of the visual field described in the earlier morphological and clinical studies. 


\subsection{Functional Differences}

\subsubsection{Animal Data}

Visually guided behavior was examined in cats in which one eye was rotated surgically between 9 and 15 days of age (Presson et al. 1983). Perimetry testing was performed when the cats were 6 months of age. The visual fields of the rotated eyes were abnormal, and regardless of the direction of eye rotation the cats appeared to be blind in the contralateral lower visual quadrants. This surprising finding, which was independent of the direction of rotation, could not be explained by the authors with any theory of rewiring of the visual inputs to the superior colliculus or the visual cortex.

In a study on visual neglect caused by section of the midbrain commissures in the cat, Matelli et al. (1983) demonstrated that after sections of the commissures between the superior and inferior colliculi the lesioned cats showed a pronounced inattention for stimuli presented in the upper visual field. They also lacked exploratory head movements toward the upper visual field. In contrast, a lesion of the posterior commissure resulted in a neglect of visual objects below the horizontal meridian (Matelli et al. 1983).

In a study on visually guided pecking in the pigeon using high-speed cinematography, Goodale (1983) determined where on the retina the visual target fell during the complex sequence of pecking. During periods of fixation, the target was located in the binocular visual field which corresponds to the red area of the upper temporal retina.

In behavioral experiments on pigeons, different spectral sensitivity functions were obtained by Martin and Muntz (1979) in the upper and lower visual fields: For short wavelengths below $550 \mathrm{~nm}$ the upper parts of the retina corresponding to the red area was found to be less sensitive than the lower parts of the retina. A similar shift of the pigeon spectral sensitivity toward longer wavelengths in the lower visual field was reported by Nuboer and Wortel (1985). This shift results in an improved discrimination capability of the animal for red and brown stimuli occurring on the ground. The physiological basis for the different spectral sensitivity of the upper and lower retina is the different regional distribution of the red oil droplets in the inner limbs of the pigeon retinal photoreceptors (see Sect. 6.1.1.1).

The pigeon visual system is also capable of detecting the plane of polarized light (Delius et al. 1976), and this enables the bird to discriminate skylight polarization. The pattern of polarization of skylight is correlated with the position of the sun, and thus gives important cues for orientation. Both behavioral and ERG data suggest that the ability to detect the plane of polarized light is associated more with the lower retina (Delius et al. 1976). These findings appear ecologically meaningful, since skylight comes from above the animal, and mainly the lower retina needs this functional advantage. 


\subsubsection{Human Data}

\subsubsection{Motor Reaction Time to Visual Stimuli}

It has long been known that there are functional differences between retinal areas in performance of a reaction time task. A widely distributed textbook on experimental psychology mentions in its first edition in 1938 that the reaction time to visual stimuli is shorter when they are presented to upper retinal areas than when they are presented to lower retinal ${ }^{*}$ reas (Woodworth 1938 , p. $327-328$ ). In later editions of the same book these findings on differences between upper and lower retinal areas are omitted (Woodworth and Schlossberg 1955). Obviously, by that time the functional differences between the upper and lower retinal areas had become too well known to need further discussion.

In most studies, regional retinal differences were reported for stimuli at locations along the horizontal meridian. In has been established that reaction times are shorter to stimuli falling onto nasal than onto temporal retinal areas (Hall and von Kries 1879; Poffenberger 1912; Rains 1963).

Hall and von Kries (1879) investigated the reaction time to stimuli along the horizontal and the vertical meridian. Their data obtained at $30^{\circ}$ and $60^{\circ}$ eccentricity are summarized in Fig. 44. Central retinal areas yield the shortest reaction times, and differences are seen between nasal and temporal and between upper and lower retinal areas. The average reaction times were significantly shorter in upper than in lower retinal areas: $34 \mathrm{~ms}$ at $30^{\circ}$ eccentricity and $46 \mathrm{~ms}$ at $60^{\circ}$ eccentricity.

Differences in the same direction were reported by Payne (1965), who investigated the reaction times at $15^{\circ}$ eccentricity in two subjects. Cocito et al. (1977) showed that the upper/lower differences depended on the spatial frequency of the stimuli used: With $1 \mathrm{c} / \mathrm{deg}$ stimuli, upper retinal areas yielded significantly shorter reaction times than lower retinal areas, whereas with $3 \mathrm{c} / \mathrm{deg}$ stimuli the authors re-

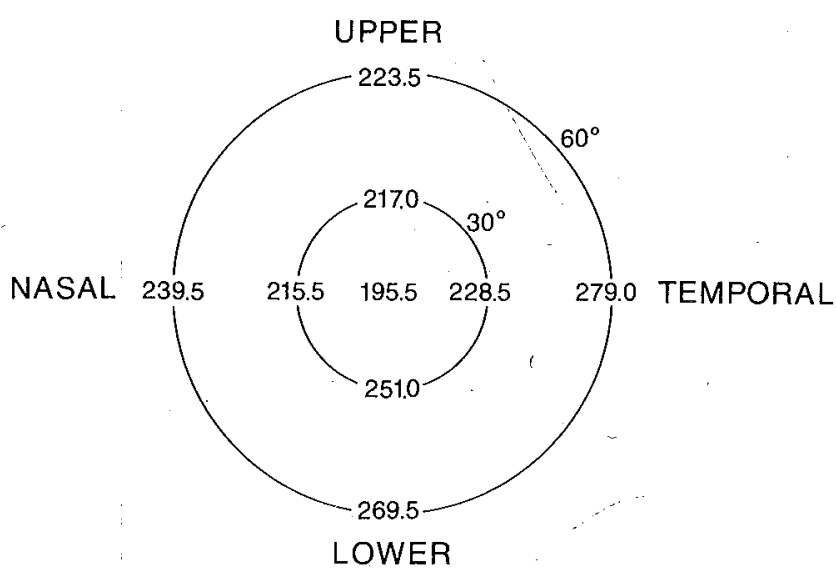

Fig. 44. Distribution of motor reaction times across the retina. Numbers indicate the mean reaction time in milliseconds averaged over two observers. (Drawn after data of Hall and von Kries 1879) 
ported the same tendency but without statistical significance. Unstructured visual stimuli were used by Magrotti et al. (1980), who found significantly shorter reaction times for upper than for lower retinal stimuli at $20^{\circ}$ eccentricity but not at $5^{\circ}$ eccentricity.

Lichtenstein and White (1961) derived equations which permitted establishment of a relation between motor reaction time to stimuli presented in various retinal locations and the local densities of the photoreceptors measured by $\emptyset_{\text {sterberg }}$ (1935). Basically, these equations show that reaction time can be considered an inverse function of photoreceptor densities in the retina.

\subsubsection{Temporal Sensitivity}

As described in Sect. 5.1 our results on critical flicker fusion frequency (CFF) and double flash discrimination (DFD) are in agreement with earlier studies. The visual field plot by Hylkema (1942) illustrated that upper and nasal retina areas had higher temporal resolutions than lower and temporal retinal areas. However, Hylkema investigated the vertical meridian much further out in the periphery (at $25^{\circ}$ eccentricity), where he obtained statistically significant differences between the upper and lower areas $[t=2.14, p<0.05$ computed from Hylkema's data (1942, p. 187)]. In Hylkema's data the lower retinal CFF reached a mean value of $96.9 \%$ of that of the upper retinal CFF. Our results show that there are significant differences in temporal resolution capacity even in perifoveal retinal areas. A comparison of the data in the published literature reveals conflicting results concerning regional differences of CFF values. Phillips (1933) measured the CFF along the diagonal meridians from $10^{\circ}$ to $40^{\circ}$ in steps of $10^{\circ}$. His data show a tendency of the upper retina areas to have higher CFF values than the lower retinal areas, while the nasal-temporal differences appear not to be significant (Phillips 1933, Fig. 2). Kleberger's (1955) data from 30 normal subjects measured at $10^{\circ}, 20^{\circ}, 30^{\circ}$ and $40^{\circ}$ along the vertical, horizontal and diagonal meridians show no consistent differences between upper and lower retinal areas, and nasal retinal areas have an inconsistent superiority over the corresponding temporal areas. Contrary to this finding, Hylkema (1942) described at $25^{\circ}$ eccentricity a higher $\mathrm{CFF}$ in the temporal retina which is thought to compensate for the nasal area of the blind spot in the opposite eye. Beyond $30^{\circ}$ eccentricity, a nasal superiority was found (Hylkema 1942). The findings of a superiority of nasal over temporal perifoveal retinal areas, presented in Sect. 5.1, are in line with an ecological interpretation suggesting a greater functional importance of the temporal visual field, where the detection of peripherally moving and approaching targets is achieved. The nasal superiority is also reflected in the regional distribution of photoreceptors in the human retina (Østerberg 1935).

Our measurements were also expressed as relative luminance threshold values (see Fig. 38). CFF drops systematically with decreasing luminance of the test field (Brown 1965; Shickman 1981), and the mean CFF differences between the upper and lower retinal areas corresponded to $0.244 \log$ filter density units. This means that the lower retinal areas needed a light intensity about 1.75 times higher in order to achieve the same temporal resolution as the corresponding upper retinal areas. 


\subsubsection{Visual Acuity and Contrast Sensitivity}

Visual acuity appears to be directly correlated with the regional distribution of photoreceptors across the retina. Aubert and Förster (1857) investigated the performance of two subjects in a two-point discrimination task. Reanalyzing their data (Aubert and Förster 1857, Tạble 4) along the vertical meridian yields a significantly better performance for the upper than the lower retinal areas. In the upper retina the two-point discrimination was successful much further out in the periphery than in the lower retina. Lantolt and Hummelsheim (1904) reported a replication of these experiments. These authors too found a superiority of upper over lower retinal areas (see Figs. 198 and 199 of Landolt and Hummelsheim 1904). They also mentioned the influence of the size of the target used on the determination of the visual field. For upper and nasal areas the target size had relatively little influence, whereas in the temporal and lower visual field the use of different target sizes for perimetry yielded different extensions of the visual field (Landolt and Hummelsheim 1904, p. 543).

Wertheim (1894) used grating stimuli to determine visual acuity of different retinal areas and found a higher visual acuity of the upper retina only beyond $20^{\circ}$ eccentricity (Fig. 5 and Table on p. 182 of Wertheim 1894). In addition, he reported that the decay in visual acuity from the fovea toward the periphery was steepest over the lower retinal areas.

Landolt rings of high contrast were used by Low (1943) and by Millodot and Lamont (1974). Low (1943) investigated the visual acuity of 100 subjects and observed a better performance for upper retinal areas than for the corresponding lower retinal areas at $30^{\circ}$ eccentricity. Millodot and Lamont (1974) mapped in three subjects the visual acuity along the vertical meridian in steps of $5^{\circ}$ from the fovea to $40^{\circ}$ in the periphery. They found a consistent superiority of the upper hemiretina over the lower hemiretina at locations beyond $5^{\circ}$ eccentricity. The disagreement in these early studies concerning where exactly the differences in visual acuity between upper and lower retinal areas become detectable can be accounted for by the different stimulus parameters (e. g., visual targets, luminances, contrast) used in those studies.

Contrast sensitivity was measured in ten subjects between 61 and 71 years of age in the center and at $10^{\circ}$ eccentricity above or below the fovea, using static and dynamic sinusoidal gratings, by Lundh et al. (1983). Similar to our results (Sect. 5.2) the fovea showed the highest contrast sensitivity, followed by the upper and then the lower hemiretina. These data, obtained further out toward the periphery than ours, are in good agreement with the findings of Wertheim (1894) and Millodot and Lamont (1974).

The contrast sensitivity functions of the perifoveal retinal regions at $5^{\circ}$ eccentricity presented in Sect. 5.2 showed a consistent superiority of the upper over the lower retinal areas, and statistically significant differences in contrast sensitivity were observed at all spatial frequencies. Both the differences in maximal contrast sensitivity, which occurred at higher spatial frequencies for upper than for lower retinal areas, and the overall differences between the contrast sensitivity functions suggest a general functional difference between upper and lowér perifoveal retinal areas which is independent of individual spatial frequency channels. Conventional visual acuity determined from these data showed large differences between the upper and lower retinal areas investigated (see Fig. 39B). 
The basis for visual acuity is believed to be reflected in the mosaic of the photoreceptors in the retina, and several authors have compared the data on visual acuity with the local morphological characteristics of the retina. From these studies it was concluded that the densities of the photoreceptors or the ganglion cells in the retina show a satisfactory high correlation with the human visual acuity in various parts of the visual field (ten Doesschate 1946; Drasdo 1977; Dawson and Maida 1984). This is supported by the fact that the acuity findings presented are in agreement with the anatomical data of the human retina (see Sect. 6.1.2.1).

\subsubsection{Perception}

In a study on figural aftereffects, Freeman (1964) investigated in 12 subjects the influence of retinal stimulus location on the size of the effect. Aftereffects were obtained only when the stimulus was presented below the horizontal meridian, and as eccentricity increased, the magnitude of the aftereffect decreased. There was no statistically significant interaction between amount of eccentricity and direction on the retina (above or below the horizontal meridian), and Freeman (1964) concluded that the effect of direction of eccentricity was a general one, independent of the amount of eccentricity. This finding suggests a larger sensitivity of the human upper visual system. In a similar line, Jampolsky (1978) reported results on visual afterimages: When equal stimulus lights fall on the upper and lower retina, the perception of an afterimage on the upper retina is dominant over that of the lower retina.

The direction of the light source is -important for visual perception: Changes in depth perception of convex and concave objects occur depending on the direction of light. When a picture showing indentations and bumps is inverted, indentations and bumps reverse. Our visual system is accustomed to light coming from above, and the shading gives information on convexity or concavity. This is the basis for the convex-concave illusion (cf., Teuber, 1974).

An interesting anisotropy between the upper and lower retinal systems was reported for stereoscopic depth perception. Breitmeyer et al. (1975) investigated the detection duration thresholds for dynamic random dot stereograms. They found that stimuli with uncrossed disparities (i. e., when the target was located behind the fixation plane) were detected at consistently shorter durations when presented to the lower hemiretina than when presented to the upper hemiretina. For crossed disparity stimuli (i. e., for targets closer to the subject than the fixation plane) the preference was reversed, with a superiority of the upper hemiretina. Similarly, the spatial resolution for uncrossed disparities was found to be better in the lower retina, and that for crossed disparities was better in the upper retina. Thus, the upper and the lower hemiretina have different spatiotemporal response properties; the upper retina is more efficient with stereoscopic stimuli nearer than the fixation point, the lower retina more sensitive for depth stimuli which are farther away. These findings can be interpreted in an ecological framework. Some elaborate motor functions like tool handling rely on the processing of visual information in the closer lower visual field, while more distant visual stimuli occur in the upper visual field. In addition, Murray et al. (1983) reported that the detection of movement measured at $20^{\circ}$ eccentricity was better in the lower than in the upper visual field (Fig. 4 of Murray et al. 1983). 
In a study on attention and visual search using a "contingent-display technique," Prinz (1984) described differences in detection performance between visual targets presented above or below the fixation point. A vertical asymmetry was found for the detection of an easy target (the letter D) but not for a difficult target (the letter $Z$ ). Visual targets below the fixation point could be detected further out in the periphery than targets above the fixation point, and the results were interpreted as an interaction between attention and sensitivity across the visual field. The retinal areas studied were very close to the fovea (about $3^{\circ}$ above or below the horizontal meridian), and these data fit well with our results obtained in a simple pattern discrimination experiment where digits presented either $5^{\circ}$ above or $5^{\circ}$ below the fixation point were significantly better discriminated by the upper retina (see Sect. 5.3).

\subsubsection{Evoked Potentials}

The amplitudes of the evoked potential for stimulation with a spot of $2.5^{\circ}$ diameter presented at various points along the horizontal and vertical meridian up to an eccentricity of $30^{\circ}$ were described by Copenhaver and Perry (1964), who published data obtained from one trained observer. Evoked potential amplitudes were in general larger for stimuli presented to upper retinal areas at all locations examined except at $5^{\circ}$ eccentricity (Fig. 2 of Copenhaver and Perry 1964).

Eason et al. (1967) reported that potentials evoked by flash stimuli have larger amplitudes and shorter latencies when presented to the upper than to the lower hemiretina. These findings were consistent in all three subjects examined.

For checkerboard reversal stimuli, Lehmann and Mir (1976) found a mean latency difference of $23 \mathrm{~ms}$, and an extreme case with a latency difference of $34 \mathrm{~ms}$ was reported by Lehmann et al. (1977).

Lehmann and Skrandies (1979) obtained a mean latency difference between upper and lower retinal areas of $14.3 \mathrm{~ms}$ for a checkerboard reversal frequency of 1.6 reversals per second, and a mean difference of $11.2 \mathrm{~ms}$ for a reversal frequency of 3.2 reversals per second. In addition, for upper hemiretinal stimuli the latencies were significantly shorter for the smaller reversal frequency, but no differences were observed for the lower hemiretina. This indicates that the upper and lower retinal systems may respond independently to a change of stimulus parameters. Lesèvre (1972) and Lesèvre and Joseph (1979) studied the topography of VEPs using chronotopograms recorded from longitudinal chains of electrodes. For lower hemiretinal stimuli the major component was located occipitally below the inion, and thus the component was missed in some recordings (e. g., Fig. 10 of Lesèvre 1972). When the lower hemiretinal response could be identified, the component latency was delayed by about $10 \mathrm{~ms}$ compared to that of the upper retina (Lesèvre and Joseph 1979).

The interpretation of potential waveshapes led Jeffreys and Smith (1979) to the conclusion that the main difference between potentials evoked by upper and lower retinal stimuli was a difference in scalp distribution. Using brief onset/offset stimulus presentations, these authors reported a polarity inversion between upper and lower evoked components. Since the latencies investigated were not identical, and owing to the complexity in interpretation of potential waveshape data (outlined in Sect. 2), one has some reservations as to the interpretation of the data. Lehmann et al. (1977) have shown that polarity inversions may be erroneously 
concluded when information on the zero voltage baseline is disregarded. The selection of appropriate electrode positions may introduce additional problems for the interpretation of evoked brain activity. More interestingly, Jeffreys and Smith (1979) presented in their Table 1 latency data which show consistently shorter component latencies for upper than lower retinal stimuli. This difference averages $8.3 \mathrm{~ms}$ and is in the same order as our latency differences obtained from topographical scalp field data (see Sect. 3 ).

Statistically significant latency differences between components evoked by stimulation of upper and lower retinal areas were also demonstrated with different presentation modes: Skrandies et al. (1980) found a mean latency difference between upper and lower retinal stimuli of $11 \mathrm{~ms}$ for pattern onset, of $8 \mathrm{~ms}$ for reversal, and a nonsignificant difference of $3 \mathrm{~ms}$ for offset. Further supporting evidence from similar experiments is reported in Sect. 3.2.2.

In a related study, Kriss and Halliday (1980) showed that for lower retinal stimuli a rather broad positive peak was observed around $120 \mathrm{~ms}$ while upper retinal stimulation yielded a generalized positivity at $100 \mathrm{~ms}$.

Kimura and Tsutsui (1981) found in ten subjects a mean latency difference of $44 \mathrm{~ms}$ for grating stimuli presented either at $15^{\circ}$ above or $15^{\circ}$ below the fixation point. This difference was statistically significant.

A large subject population was examined by Kuba et al. (1982) using a checkerboard reversal stimulus. Based on the data of 60 subjects, they reported a statistically significant mean latency difference of $17 \mathrm{~ms}$ between upper and lower retinal stimulation.

Using steady-state evoked potentials, Yanashima (1982) described larger amplitudes evoked by upper retinal than by lower retinal checkerboard reversal stimuli over the occipital areas. Yanashima (1982) also reported a phase difference of about $180^{\circ}$ for the $12 \mathrm{~Hz}$ component which corresponds to a latency difference of about $42 \mathrm{~ms}$. The distribution of the electrical activity is hard to interpret, since the electrode array did not extend to below the inion and only the absolute power of the Fourier components was analyzed. When considering the neuronal generating mechanisms (see Fig. 45), it is not surprising that Yanashima (1982) found larger amplitudes with lower retinal stimuli over anterior scalp areas: The power values are polarity-independent, and it appears conceivable that a relative negativity is more pronounced over distal scalp areas [see also Sect. 2 and Lehmann (1984) for a discussion of the scalp mapping of spectral power].

Adachi-Usami and Lehmann (1983) investigated the differences between monocular and binocular evoked potentials and found significant latency differences between upper and lower retinal stimuli in all experimental conditions. For small check sizes $\left(14^{\prime}\right.$ arc), the latency differences averaged $15.2 \mathrm{~ms}$ for monocular and $12.3 \mathrm{~ms}$ for binocular stimuli; for a check size of $56^{\prime}$ arc, the monocular differences between upper and lower retinal stimuli were $13.2 \mathrm{~ms}$ and the binocular differences were $15.5 \mathrm{~ms}$.

Skrandies (1984) reported for vertical grating stimuli of $2.3 \mathrm{c} / \mathrm{deg}$ a mean latency difference between components evoked by upper or lower retinal stimulation of $5.4 \mathrm{~ms}$. These data were obtained in a population of 12 healthy adults and the latency differences were highly significant.

All these human evoked potential results are in good agreement with the findings presented in Sect. 3, where shorter component latencies were obtained for scalp 

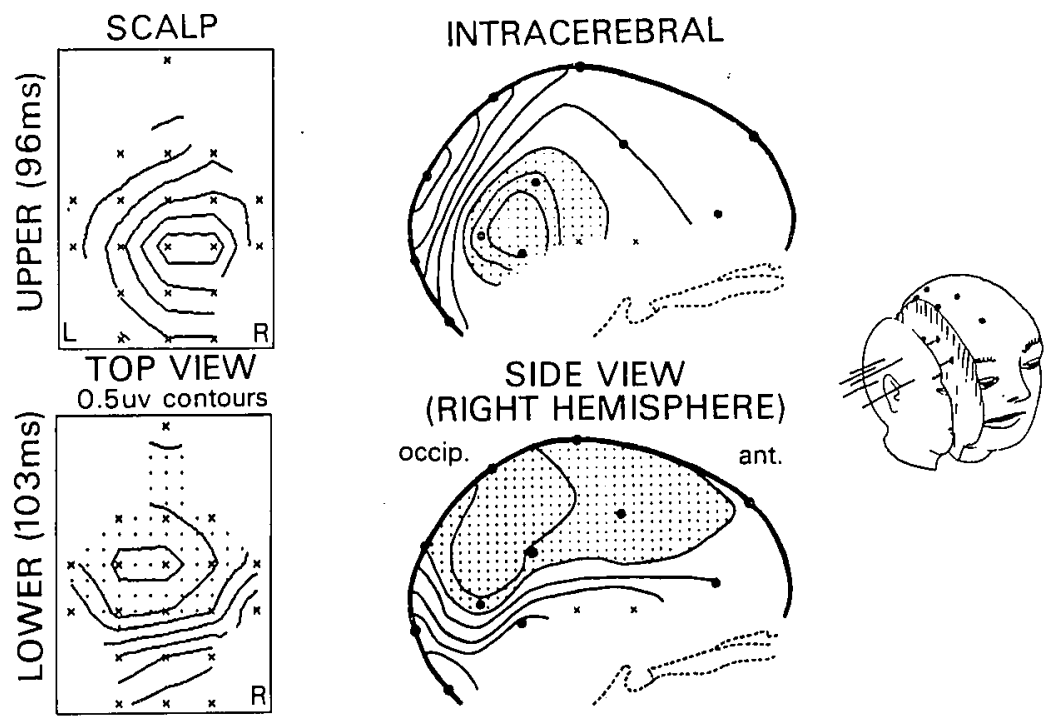

Fig. 45. Intracerebral and scalp potential fields evoked by upper or lower hemiretinal checkerboard reversal stimuli recorded simultaneously in 48 channels ( 20 scalp and 28 intracranial electrodes). Intracerebral recording sites are marked by dots, and these potential fields are located in the parasagittal plane $3 \mathrm{~cm}$ from the midline (right). (From Lehmann et al. 1982; by permission of Raven Press, New York)

field data evoked by upper retinal stimuli. In addition to the latency differences there are also differences in scalp location of the major evoked components. The general finding that components evoked by upper retinal stimulation are located more anteriorly on the scalp than components evoked by lower hemiretinal stimuli agrees well with the underlying physiologic projection of the visual field onto the visual cortex (see Sect. 6.1). Similar location differences have also been described by Lehmann et al. (1977), Lehmann and Skrandies (1979), Lesèvre and Joseph (1979), Skrandies et al. (1980), Skrandies (1984a,b), or Adachi-Usami and Lehmann (1983).

In a study on intracerebrally recorded potential fields in human patients (Lehmann et al., 1982), we have had the opportunity to study the relationship between intracranial and scalp-recorded brain activity. Figure 45 shows the simultaneously recorded intracerebral and scalp field distributions evoked by upper and lower hemiretinal checkerboard reversal stimuli at the respective component latencies. The intracranial fields in the two stimulus conditions appear to be rotated with respect to each other. According to a stereotactic atlas of the human brain (Talairach and Szikla 1967), the position of the rotation axis corresponds to the middle of the calcarine fissure level. Not only are the positions of the maxima and minima of the evoked fields different, the regions where high potential gradients are located also differ: for upper retinal stimuli the highest potential gradients occur above and for lower retinal stimuli below the level of the calcarine fissure (Fig. 45). These data, in combination with theoretical model dipole computations (Lehmann et al. 1982), illustrate that scalp topography is related to intracranial brain activity in a physiologically meaningful way. 


\subsection{Clinical Observations}

The results presented in this chapter are not intended primarily as further support for functional superiority of the upper over the lower hemiretina. It will rather be shown that the upper and lower visual systems of man are anatomically and physiologically independent to a certain degree, as reflected by the functional results discussed in Sect. 6.2.2. Independence of upper and lower visual fields becomes obvious in various pathological conditions affecting the human visual system.

Some evidence on differences between the upper and the lower retina stems from clinical experience rather than from systematic investigations: Jampolsky (1978) reported that a pathological vertical fixation disparity is observed as an adaptive process of the upper retina only, and that with dissociated vertical divergence in infantile alternating esotropias the eye deviates upward (Jampolsky 1978).

A similar observation is that in most people the eyes are quickly turned upward when the lids are forcibly closed. This reflex, known as Bell's phenomenon, may be regarded as an avoidance reaction that protects the anterior segment of the eye (i. e., the cornea and lens) as well as the upper retina from mechanical damage.

\subsubsection{Glaucoma}

Both vascular insufficiency and mechanical damage to fibers of the optic nerve have been reported to be caused by elevation of intraocular pressure (Hayreh et al. 1970; Quigley et al. 1980). Electrophysiological studies in the cat have shown that during short-term elevation of intraocular pressure the function of the retinal ganglion cells depends on perfusion pressure and not on the absolute level of intraocular pressure (Grehn and Prost 1983).

Visual field defects in glaucoma show a characteristic pattern, as described in a study on 215 patients by Gramer et al. (1982). Most field defects were found in the upper visual field; this was true for both absolute and relative defects. The authors interpret their findings as a possible indication of a small morphological difference in the routing of nerve fiber bundles of the lower retinal half which may be more susceptible to damage caused by elevated intraocular pressure. Drance (1977) described the visual fields of 12 eyes of patients with shock-induced optic neuropathy, 28 eyes of patients with low-tension glaucoma, and 35 eyes of patients with chronic open-angle glaucoma. In all three groups the field defects were located more often in the upper than in the lower visual field. In shock-induced neuropathy and low-tension glaucoma upper field defects occurred three times as often as lower field defects, and in chronic glaucoma $64.1 \%$ of the field defects were restricted to the lower hemiretina.

When the extent of the visual field is determined by perimetry, some scatter of its boundaries is always observed. Flammer et al. (1984) investigated the factors which influence such short-term fluctuations in automated static perimetry. In patients suffering from glaucoma, larger short-term fluctuations were observed in the upper half of the visual field where field defects also occurred more frequently. A similar tendency appeared in the data of the healthy subjects (see Fig. 3 of Flammer et at. 1984). The main factor for these short-term fluctuations was the 
differential light sensitivity at the respective test location. This relationship was statistically significant.

\subsubsection{Retinal Circulation}

Jones and Wilcott (1977) reported impaired dark adaptation and night vision related to physical exercise in four subjects. During exercise the speed of dark adaptation was slower in all subjects, and the threshold for night vision was about $1 \mathrm{log}$ unit higher than during a resting condition. On testing the dark adaptation of peripheral retinal areas (at $10^{\circ}$ nasal and up or down) the authors found that in the lower visual fields the decrease in the speed of dark adaptation was even greater than in the upper visual fields, and a markedly elevated threshold for night vision was present in all subjects during the entire exercise interval. The threshold was elevated by $2 \log$ units or more during the first 15-20 min, and the elevation remained at a level of about $2 \log$ units during the 60 min-period of exercise. Impaired night vision had not been reported during moderate exercise, and Jones and Wilcott (1977) concluded from their data that the lack of increased ophthalmic arterial pressure might result in a minute paucity of blood in upper retinal areas. This subtle circulatory change as a direct physiological effect may be reflected in the altered dark adaptation.

\subsubsection{Epilepsy}

Some pattern-sensitive epileptics are specifically responsive to grating patterns of certain orientations. Soso et al. (1980) investigated two pattern-sensitive epileptics stimulated by grating patterns of different orientations presented in the upper or lower visual hemifield. Most EEG spike discharges were found during stimulation of the upper visual field, while the lower visual field yielded only very few spike discharges (see Table 1 of Soso et al. 1980). These findings show that input received via the lower hemiretina has a much higher seizure-facilitating capacity than stimuli presented to the upper hemiretina, supporting the notion that the two visual hemifields may behave as functionally independent systems.

\subsubsection{Cerebral Hypoxia}

Zihl and von Cramon (1986) described amblyopic visual field defects in patients suffering from chronic cerebral hypoxia. Most of their patients (eight of ten) showed alterations in the lower half of their visual fields. These defects comprised either scotoma or areas with greatly reduced form and color vision. There were, however, no pathophysiological peculiarities in these patients which could account for this asymmetrical distribution of visual field defects (Zihl, personal communication). These observations demonstrate a relative independence of the upper and lower visual hemifields which presumably originates at a cortical level. 


\section{Summary and General Conclusions}

In a series of experiments on human vision I investigated electrophysiological and functional differences between the upper and lower halves of the visual world. The methodological aspect of the work was concerned with adequate biophysical analysis of the electrical brain fields, starting with animal experiments in which volume conduction of brain activity was examined by correlating single unit data with mass activity and going on to the study of the surface-recorded electrical fields of the human brain where multichannel topographical mapping was performed. The aim of these studies was the extraction and statistical analysis of reference-independent descriptors of electrical brain field configurations as recorded from the scalp. Putative neuronal generator sites were described and correlated with earlier results of intracranial evoked potential recordings in human patients.

Since there are fundamental ecological differences between the upper and lower parts of the visual field of man, most of whose objects of interest appear below the horizon, I conducted several series of experiments addressing the question of possible electrophysiological and functional differences in the processing of information in the upper and lower visual fields. The following electrophysiologically ascertained systematic differences support the notion of a functional superiority of the upper hemiretinal system:

1. The component latencies of electrical brain activity evoked by stimuli presented to lower hemiretinal areas were longer than those evoked by upper retinal stimuli, and the scalp topography was consistent with the general neuroanatomical knowledge. These results were found in various subject populations and were independent of the visual targets and the presentation modes: checkerboard and grating stimuli, and pattern onset, reversal, and offset presentations yielded similar differences in brain activity evoked by upper vs lower visual stimuli.

2. The pattern electroretinogram showed similar differences as did the cortical evoked potential with stimulation of upper vs lower retinal areas. These data were obtained in multichannel recordings in which electrical activities originating in the retina and in the visual cortex were separated by topographical methods.

3. The standing potential of the human eye is affected by light stimulation: in electro-oculographic experiments the light response reflecting the interaction between photoreceptors and the retinal pigment epithelium was found to be significantly larger when upper retinal areas were stimulated. In addition, upper retinal areas were more sensitive to changes of the test light intensity.

In psychophysical experiments I was able to demonstrate functional correlates of the electrophysiological findings:

1. Temporal resolution as defined by critical flicker fusion was higher when visual stimuli occurred in the lower visual field. Experiments on double flash discrimination confirmed this finding.

2. Contrast sensitivity was higher for sinusoidal grating patterns presented in the lower than in the upper visual field. These differences were independent of single spatial frequency channels. The conventional visual acuities derived from these data were higher for the upper than the lower retina. 
3. In a pattern discrimination task the subjects were better at discriminating stimuli presented on the lower half than on the upper half of a video screen.

Thus, the global superiority of the upper visual system appears to extend from the first step in visual information processing in the retina to higher perceptual and cortical mechanisms, and these functional differences may also have implications for higher visual information processing in conditions involving a man-machine interface.

All differences found were in the same order of magnitude (around 10\%), and it is not possible to determine whether the electrophysiological differences observed at later stages (e.g., evoked potential latencies) are directly caused by the differences at earlier stages of the visual system. The data reviewed in Sect. 6 show that anatomical differences between the upper and lower hemiretinal systems exist both at the retinal level (e.g., regional differences in the distribution of photoreceptors) and in the visual cortex, where the lower visual field has a larger representation, and the consistent electrophysiological and functional differences discussed in this chapter may be interpreted as a general system difference.

The differences between the upper and lower hemiretinal systems of man are probably related to the fact that the upper and lower halves of the outer visual world are of different importance for the organism. The function of perception is the acquisition of useful information about the environment. Hence, brain mechanisms of visual information processing must be adapted and optimized to the specific environment of the organism. Information about the outer world in turn enables the animal to respond adequately to sensory stimuli as well as to complex stimulus configurations. Contrary to simplifying laboratory conditions, in which light spots, isolated contours, or near-threshold visual stimuli are presented, in real life the processing of complex patterns of physical stimuli also takes into account the meaningfulness of the stimuli in the outer world. The environmental parameters determine all perceptual mechanisms and capabilities [see also Gibson (1966) for a discussion of such an ecological approach to perception]. Survival requires the ability of the organism to select perceivables on the basis of their importance, and thus the aim of the sensory systems must be to tell the organism what is out there. Sensory information is compared with memory contents in order to achieve adequate interpretations of the environmental information and to adjust the systems' functional state via the orienting response to a condition which optimally permits the selection and execution of adequate reactions to the stimulus configuration.

In addition, the ability to concentrate on certain features of the constant information flow is important. The organism must be able to select useful, relevant information while ignoring other stimuli, and it must also be alert to any changes in the stimulus configurations.

There are pronounced differences between species, even in the gross anatomy of sensory systems' which reflect differences in phylogenesis and habitat. For instance, prey and predator differ: The rabbit's eyes are located laterally on the head, yielding a panoramic field of view. Thus, the animal is able to detect enemies approaching from any direction. For hunting animals the binocular field of view is more important, and fox and wolf have frontal eyes with a smaller total visual field but a relatively large binocular field. Ecological factors and evolution 
press for optimal adaptation of the sensory systems to the environment (Gibson 1966).

The differences in significance between the upper and lower visual fields fit nicely into such an ecological framework. Stimuli occurring above or below the visual horizon typically carry different meaning and have a different degree of importance for the organism. For most mammals, the upper visual field appears to be less important than the lower. The upper visual field includes mainly vegetation and sky and contains less texture than the lower visual field, most things of interest are located in the lower hemisphere of the visual world. The horizon is the natural optical boundary between sky and ground. Most organisms are capable of detecting verticality, which is also defined by gravity, and they orient according to the difference between up and down.

The basic general difference between the upper and lower visual hemipheres is that direct light always comes from above. Sunlight is very intense, and therefore the lower hemiretina, which is less sensitive than the upper, is well adapted to the light intensity. The convex-concave illusion described in Sect.6.2.2.4 illustrates that our visual system always assumes that light comes from above.

Light coming from the lower visual field is reflected from object surfaces and is less intense. Prey or enemies of most mammals which do not have birds as predators occur in the lower visual field. For man also, most objects of interest are below the horizon, and the upper visual system must analyse fine textures and the details of the visual world. Therefore, the upper visual system is endowed with higher sensitivity and with better spatial and temporal resolution capacities than the lower visual areas. In addition, the coordination of man's hands in the lower binocular visual field is guided by vision and locomotion relies mainly on information from the lower visual hemisphere, and there are also differences in stereoscopic information processing between the upper and lower visual fields, as reviewed above.

There are meaningful exceptions to this upper visual system superiority in some species: The pigeon retina shows different pigmentation in its upper and lower parts, making the lower retina more sensitive for blue light. In addition, mainly the lower retina of the pigeon is able to detect the plane of polarized light. This enables the animal to orient according to the polarization pattern of the skylight coming from above.

In small animals like the rat, mouse, or frog, evidence has been obtained of asymmetry in midbrain structures of the visual system favoring the upper visual hemisphere. The upper visual field may carry important information for these animals. Enemies of the mouse and the rat may approach from above, and frogs have to catch flies in the upper binocular field of view.

The reviewed human anatomical and clinical data show that the upper and lower retinal halves may be considered as physiologically independent systems. In support of this conjecture, functional differences between upper and lower retinal areas were found in human psychophysical experiments which show that visual acuity, temporal sensitivity, and pattern discrimination are better with stimuli presented in the lower than in the upper visual field.

The data obtained in electrophysiological experiments correlate well with the observed functional differences between the upper and lower visual systems. The electrical fields of the human brain recorded from the intact scalp were shown to 
be directly related to the underlying neuronal processes, and topographical analyses of these potential fields demonstrated significant differences between the processing of upper and lower retinal stimulus material. Consistent differences in scalp topography and in component latency of the evoked components are found in various stimulus conditions and subject populations.

In addition, the differences between upper and lower areas can already be demonstrated at the level of the retina. The functional superiority of the upper visual system thus appears to extend from the first step in visual information processing in the retina to higher, complex perceptual mechanisms, as reflected by the better performance in pattern discrimination tasks where meaningful stimulus material is used.

Moreover, during ontogenetic development there is an interaction between the functional aspects of information processing and the organization of the sensory systems. In cats it has been shown that early visual experience alters the physiological responses of the visual system. As a consequence of early light deprivation during a sensitive period, the number of cortical neurons that later in life can be influenced by light declines (Hubel and Wiesel 1970b). Hirsch and Spinelli (1970) studied cats which were raised from birth with one eye viewing vertical lines and the other eye viewing horizontal lines, and they found that the receptive fields of cortical neurons were predominantly oriented according to this early experience. These studies illustrate that functional neuronal connections can be selectively modified by environmental stimulus configurations. Similarly, Annis and Frost (1973) described orientation anisotropies in human visual acuity of adult subjects. These anisotropies appeared to be directly related to the early visual environment in which the subjects had been raised.

Perceptual capability is determined phylogenetically and ontogenetically both by anatomical factors and by the structure of the environment. Thus, sensory systems are optimally adapted to the habitat, and deal with the demands of specific aspects of visual information processing. In the case of the division of the outer world into an upper and a lower half, it can be argued that phylogenetically, human prey and enemies occurred mainly in the lower parts of the visual field, and therefore improved performance of the human upper visual system was advantageous.

Acknowledgements. The experiments presented in this text were performed in the course of the past few years, and several persons contributed to different aspects of this final summary. First of all, I wish to thank Professor Dr. E. Dodt for his continuing support of my work and Professor Dr. D. Lehmann for many fruitful and inspiring discussions and his close cooperation on the topography of electrical brain activity. Professor Dr. H. Wässle and Dr. L. Peichl gave useful hints on anatomical findings in the retina. Professor Dr. C. Baumann, Professor Dr. E. Dodt, Professor Dr. D. Lehmann, and Dr. D. Brandeis read a first draft of the manuscript and made valuable suggestions for this final version. Thanks are also due to Dr. A. Raile, who helped with the data collection in some of the experiments described in chapter 4.2; to Dr. O. Ludwig for his advice concerning statistical problems; and to Mrs. H. Schneider, who prepared most of the figures. 


\section{References}

Adachi-Usami E, Lehmann D (1983) Monocular and binocular evoked average potential field topography: upper and lower hemirethnal stimuli. Exp Brain Res 50:341-346

Aebersold H, Creutzfeldt OD, Kuhnt U and Sanides D (1981) Representation of the visual field in the optic tract and optic chiasm of the cat. Exp Brain Res 42:127-145

Afanador AJ, Andrews CE (1978) Rod and cone contribution to the EOG ratio. Am J Optom 55:101-107

Ali MA, Klyne MA (1985) Vision in vertebrates. Plenum New York

Alpern M (1972) Eye movements. In: Jameson D, Hurwich LM (eds) Handbook of sensory physiology, vol VII/4. Springer, Berlin Heidelberg New York, pp 304-330

Annis RC, Frost B (1973) Human visual ecology and orientation anisotropies in acuity. Science 182:729-731

Arden GB, Kelsey JH (1962a) Changes produced by light in the standing potential of the human eye. J Physiol 161, 189-204

Arden GB, Kelsey JH (1962b) Some observations on the relationship between the standing potential of the human eye and the bleaching and regeneration of visual purple. $J$ Physiol 161, 205-226

Arden GB, Carter RM, Hogg C, Siegel IM, Margolis S (1979) A gold foil electrode: extending the horizons for clinical electroretinography. Invest Ophthalmol 18, 421-426

Armington JC (1968) The electroretinogram, the visual evoked potential, and the arealuminance relation. Vision Res $1968,8,263-276$

Armington JC (1974) The electroretinogram. Academic, New York

Aschoff U (1981) Skotopische und photopische Anteile der Hell- und Dunkelschwingung im Elektrookulogramm. Dev Ophthalmol 4, 149-166

Aubert H, Förster R (1857) Beiträge zur Kenntnis des indirecten Sehens. I. Untersuchungen über den Raumsinn der Retina. Arch Ophthalmol 3, 1-37

Baizer JS, Maguire WM (1983) Double representation of lower visual quadrant in prelunate gyrus of rhesus monkey. Invest Ophthalmol Vis Sci $24,1436-1439$

Bartlett NR, Sticht TG, Pease VP (1968) Effects of wavelength and retinal locus on the reaction time to onset and offset stimulation. $J$ Exp Psychol 78, 699-701

Basler A (1911) Über die Verschmelzung von zwei nacheinander erfolgenden Lichtreizen. Pflügers Arch 143, 245-251

Bennet-Clark HC (1964) The oculomotor response to small target replacements. Optica Acta 11, 301-314

Berger $H$ (1929) Über das Elektrenkephalogramm des Menschen. 1. Mitteilung. Arch Psychiat Nervenkrankh 87, 527-570

Bilge M, Bingle A, Seneviratne KG, Whitteridge D (1967) A map of the visual cortex in the cat. J Physiol 191, 116P-118P

Bishop PO, Kozak W, Vakkur GJ (1962) Some quantitative aspects of the cat's eye: axis and plane of reference, visual field co-ordinates and optics. J Physiol 163, 466-502

Bjaalie JG (1985) Distribution in areas 18 and 19 of neurons projecting to the pontine nuclei: a quantitative study in the cat with retrograde transport of HRP-WGA. Exp Brain Res 57, 585-597

Bjaalie JG, Brodal P (1983) Distribution in area 17 of neurons projecting to the pontine nuclei: a quantitative study in the cat with retrograde transport of HRP-WGA. J Comp Neurol 221:289-303

Bodis-Wollner I, Diamond SP (1976) The measurement of spatial contrast sensitivity in cases of blurred vision associated with cortical lesions. Brain 99, 695-710

Braddick D, Campbell FW, Atkinson J (1978) Channels in vision: basic aspects. In: Held R, Leibowitz HW, Teuber H-J (eds) Handbook of sensory physiology, vol 8. Springer, Berlin Heidelberg New York, pp 3-38 
Breitmeyer B, Julesz B, KropfI W (1975) Dynamic random-dot stereograms reveal up-down anisotropy and left-right isotropy between cortical hemifields. Science 187, 269-270

Brettel H, Caelli T, Hilz R, Rentschler I (1982) Modelling perceptual distortion: amplitude and phase transmission in the human visual system. Hum Neurobiol 1, 61-67

Brindley G, Lewin WS (1968) The sensations produced by electrical stimulation of the visual cortex. J Physiol 196, 479-493

Broca P (1861) Perte de la parole. Romolissement chronique et destruction partielle du lobe anterieur gauche du cerveau. Bull Soc Anthrop (Paris) 219

Brown JL (1965) Flicker and intermittent stimulation. In: Graham $\mathrm{CH}$ (ed) Vision and visual perception. Wiley, New York, pp 251-320

Burkhalter A, Felleman DJ, Newsome WT, van Essen DC (1986) Anatomical and physiological asymmetries related to visual areas V3 and VP in macaque extrastriate cortex. Vision Res 26, 63-80

Campbell FW, Robson JG (1968) Applications of Fourier analysis to the visibility of gratings. J Physiol 197, 551-566

Caton R (1875) The electric currents of the brain. Br Med J 2, 278

Clarke PGH, Whitteridge D (1976) The projection of the retina, including the red area, on to the optic tectum of the pigeon. Q J Exp Physiol 61, 351-358

Cocito L, Favale E, Tartaglione A (1977) Asimmetrie funzionali tra emicampo visivo superiore ed inferiore nel soggetto normale. Boll Soc It Biol Sper 53, 629-633

Copenhaver RM, Perry NW (1964) Factors affecting visually evoked cortical potentials such as impaired vision of varying etiology. Invest Ophthalmol 3, 665-675

Creutzfeldt OD (1983) Cortex cerebri. Springer Berlin Heidelberg New York

Creutzfeldt OD, Kuhnt U (1967) The visual evoked potential: physiological, developmental, and clinical aspects. Electroencephalogr Clin Neurophysiol [Suppl 26]:29-41

Curcio CA, Hendrickson AE, Kalina RE (1985) Topographical distribution of human photoreceptors. Invest Ophthalmol Vis Sci 26 [Suppl]:261

Dawson WW, Maida TM (1984) Relations between the human retinal cone and ganglion cell distribution. Ophthalmologica 188, 216-221

Delius JD, Perchard RJ, Emmerton J (1976) Polarized light discrimination by pigeons and an electroretinographic correlate. J Comp Physiol Psychol 90, 560-571

Dimond SJ, Beaumont JG (1974) Hemisphere function in the human brain. Elek Science, London, p 398

Ditchburn RW (1973) Eye movements and visual perception. Clarendon, Oxford

Dodt E (1951) Cone electroretinography by flicker. Nature 168:738

Dodt E (1964) Erregung und Hemmung retinaler Neurone bei intermittierender Belichtung. Doc Ophthalmol 18, 259-274

Dodt E, Baier M (1984) Area-luminance relationship for a constant light peak of the standing potential in the human eye. Ophthalmologica 188, 232-238

Dodt E, Enroth C (1954) Retinal flicker response in cat. Acta Phys Scand 30, 375-390

Donchin E, Ritter W, McCallum WC (1978) Cognitive psychophysiology: the endogenous components of the ERP. In: Callaway E, Tueting P, Koslow SH (eds), Event-related brain potentials in man. Academic, New York, pp 349-411

Dräger UC, Hubel DH (1976) Topography of visual and somatosensory projections to mouse superior colliculus. J Neurophysiol 39, 91-101

Drance SM (1977) The visual field of low tension glaucoma and shock-induced optic neuropathy. Arch Ophthalmol 95, 1359-1361

Drasdo N (1977) The neural representation of visual space. Nature 266, 554-555

Eason RG, White CT, Oden D (1967) Averaged occipital responses to stimulation of sites in the upper and lower halves of the retina. Percept Psychophys 2, 423-425

Ehrlich D (1981) Regional specialization of the chick retina as revealed by the size and density of neurons in the ganglion cell layer. J Comp Neurol 195, 643-657 
Elenius V, Aantaa E (1973) Light-induced increase in amplitude of electro-oculogram. Arch Ophthalmol 90, 60-63

Emmerton $J$ (1983a) Functional morphology of the visual system. In: Abs M (ed), Physiology and behaviour of the pigeon, Academic, New York, pp 221-244

Emmerton J (1983b) Vision. In: Abs M (ed), Physiology and behaviour of the pigeon, Academic, New York, pp 245-266

Estevez O, Spekreijse H (1974) Relationship between pattern appearance - disappearance and pattern reversal response. Exp Brain Res 19, 233-238

Flammer J, Drance SM, Fankhausen F, Augustiny L (1984) Differential light threshold in automated static perimetry. Arch Ophthalmol 102, 876-879

Fox SS, O'Brien JH (1965) Duplication of evoked potential waveform by curve of probability of firing of a single cell. Science $147,888-890$

Freeman RB (1964) Figural after-effects: displacement or contrast. Am J Pyschol 77, $607-613$

Freeman WJ (1978) Discussion in E. Donchin: Use of scalp distribution as a dependent variable in event-related potential studies: excerpts of preconference correspondence. In: Otto DA (ed) Multidisciplinary perspectives, in event-related brain potential research. EPA, Washington, pp 501-510

Gazzaniga MS (1970) The bisected brain. Appleton-Century-Crofts, New York

Gibson A, Baker J, Mower G, Glickstein M (1978) Corticopontine cells in area 18 of the cat. J Neurophysiol 41, 484-495

Gibson JJ (1966) The senses considered as perceptual systems. Houghton Mifflin, Boston

Glickstein M, Stein J, King RA (1972) Visual input to the pontine nuclei. Science 178, $1110-1111$

Goodale MA (1983) Visually guided, pecking in the pigeon (Columba livia). Brain Behav Evol 22, 22-41

Gramer E, Gerlach R, Krieglstein GK, Leydhecker W (1982) Zur Topographie früher glaukomatőser Gesichtsfeldausfälle bei der Computerperimetrie. Klin Monatsbl Augenheilk $180,515-523$

Granit R (1947) Sensory mechanisms of the retina. Hafner, New York

Granit R (1955) Centrifugal and antidromic effects on ganglion cells of retina. J Neurophysiol 18, 388-411

Granit R, Hammond EL (1931) Comparative studies on the peripheral and central retina. $\mathrm{V}$. The sensation-time curve and time course of the fusion frequency on intermittent stimulation. Am J Physiol 98, 654-663

Greenberg JH, Reivich M, Alavi A, Hand P, Rosenquist A, Rintelmann W, Stein A, Tusa R, Dann R, Christman D, Fowler J, McGregor B, Wolf A (1981) Metabolic mapping of functional activity in human subjects with the [18-F]fluorodeoxyglucose technique. Science 212, 678-680

Grehn F, Prost M (1983) Function of retinal nerve fibers depends on perfusion pressure: neurophysiologic investigations during acute intraocular pressure elevation. Invest Ophthalmol 24, 347-353

Griff ER, Steinberg RH (1982) Origin of the light peak: in vitro study of Gekko gekko. J Physiol 331, 637-652

Groneberg A, Teping C (1980) Topodiagnostik von Sehstörungen durch Ableitung retinaler und kortikaler Antworten auf Umkehr-Kontrastmuster. Ber Dtsch Ophthalmol Ges 77, $409-415$

Grüsser O-J (1984) Face recognition within the reach of neurobiology and beyond it. Hum Neurobiol 3, 183-190

Grüsser O-J, Kapp H (1958) Reaktionen retinaler Neurone nach Lichtblitzen. II. Doppelblitze mit wechselndem Blitzintervall. Pflügers Arch 266, 111-129

Grüsser O-J, Rabelo C (1958) Reaktionen retinaler Neurone nach Lichtblitzen. I. Einzelblitze und Blitzreize wechselnder Frequenz. Pflügers Arch 265, 501-525 
Gstalder RJ, Green DG (1971) Laser interferometric acuity in amblyopia. J Pediatr Ophthalmol 8,251-256

Hall GS, von Kries J (1879) Über die Abhängigkeit der Reaktionszeit vom Ort des Reizes. Arch Anat Physiol (Leipzig) [Suppl] 1-10

Halliday AM, McDonald WI, Mushin J (1973) The visual evoked response in the diagnosis of multiple sclerosis. Br Med J 4, 661-664

Hayreh SS, Revie IHHS, Edwards J (1970) Vasogenic origin of visual field defects and optic nerve changes in glaucoma. Br J Ophthalmol 54, 461-472

Hebel R, Holländer H (1983) Size and distribution of ganglion cells in the human retina. Anat Embryol 168, 125-136

Hirsch HVB, Spinelli DN (1970) Visual experience modifies distribution of horizontally and vertically oriented receptive fields in cats. Science $168,869-871$

Hjorth B (1975) An on-line transformation of EEG scalp potentials into orthogonal source derivations. Electroencephalogr Clin Neurophysiol 39, 526-530

Holden AL, Powell TPS (1972) The functional organization of the isthmo-optic nucleus in the pigeon. J Physiol 223, 419-447

Holländer H, Bisti S, Maffei L, Hebel R (1984) Electroretinographic responses and retrograde changes of retinal morphology after intracranial optic nerve section. A quan-

titative analysis in the cat. Exp Brain Res 55, 483-493

Holm S (1979) A simple sequentially rejective multiple test procedure. Scand J Statist 6, $65-70$

Holmes G (1945) The organization of the visual cortex in man. Proc R Soc, Lond (Biol) $132,348-361$

Hubel DH, Wiesel TN (1970a) Cells sensitive to binocular depth in area 18 of the macaque monkey cortex, Nature 225, 41-42

Hubel DH, Wiesel TN (1970b) The period of susceptibility to the physiological effects of unilateral eye closure in kittens. J Physiol 206, 419-436

Hughes A (1975) A quantitative analysis of the cat retinal ganglion cell topography. J Comp Neurol 163, 107-128

Hughes A, Wässle $\mathrm{H}$ (1976) The cat optic nerve: fibre total count and diameter spectrum. J Comp Neurol 169, 171-184

Hylkema BS (1942) Examination of the visual field by determining the fusion frequency. Acta Ophthalmol 20, 181-193

Jampolsky A (1978) Unequal visual inputs in strabismus management: a comparison of human and animal strabismus. In: Symposium on strabismus. Transactions of the New Orleans Academy of Ophthalmology. Mosby, St. Louis, pp 358-492

Jeeves MA (1984) The historical roots and recurring issues of neurobiological studies of face perception. Hum Neurobiol 3, 191-196

Jeffreys DA, Smith AT (1979) The polarity inversion of scalp potentials evoked by upper and lower half field stimulus patterns: latency or surface distribution differences? Electroencephalogr Clin Neurophysiol 46, 409-415

Jewett DL, Romano MN, Williston JS (1970) Human auditory evoked potentials: possible brain stem components detected on the scalp. Science 167, 1517-1518

Jones RK, Wilcott IT (1977) Topographic impairment of night vision related to exercise. Am J Ophthalmol 84, 868-871

Julesz B (1971) Foundations of cyclopean perception. University of Chicago Press, Chicago

Kavanagh RN, Darcey TM, Lehmann D, Fender DH (1978) Evaluation of methods for three-dimensional localization of electrical sources in the human brain. IEEE Trans Biomed Eng 25, 421-429

Kimura $\mathrm{H}$, Tsutsui J (1981) Average responses evoked by moving grating pattern in the upper, central and lower visual field. Neurosci Lett 24, 295-299 
King-Smith PE (1969) Absorption spectra and function of the colored oil drops in the pigeon retina. Vision Res 9, 1391-1399

Kleberger E (1955) Untersuchungen über die Verschmelzungsfrequenz intermittierenden Lichts an gesunden und kranken Augen. III. Das normale Flimmergesichtsfeld. Graefes Arch Ophthalmol 157, 158-166

Kriss A, Halliday AM (1980) A comparison of occipital potentials evoked by pattern onset, offset and reversal by movement. In: Barber C (ed) Evoked potentials. MTP Press, Lancaster, pp 205-212

Kuba M, Peregrin K, Vit F, Hanusova I (1982) Visual evoked responses to reversal stimulation in the upper and lower half of the central part of the visual field in man. Physiol Bohemeslow 31, 503-510

Lanclau D, Dawson WW (1970) The histology of retinas from the pinnipedia. Vision Res $10,691-702$

Landis C (1954) Determinants of critical flicker-fusion threshold. Physiol Rev 34, 259-286

Landolt E, Hummelsheim E (1904) Die Untersuchung der Funktionen des excentrischen Netzhautgebietes. In: Sämisch T (ed) Grăfe-Sämisch Handbuch der gesamten Augenheilkunde, vol 4. Engelmann, Leipzig, pp 503-583

Lawden MC (1982) The analysis of spatial phase in amblyopia. Hum Neurobiol 1, 55-60

Lehmann D (1977) The EEG as scalp field distribution. In: Rémond A (ed) EEG informatics. Elsevier, Amsterdam, pp 365-384

Lehmann D (1984) EEG measurement of brain activity: spatial aspects, segmentation and imaging. Int J Psychophysiol 1, 267-276

Lehmann D, Julesz B (1978) Lateralized cortical potentials elicited by dynamic random dot stereograms. Vision Res 18, 1265-1271

Lehmann D, Mir Z (1976) Methodik und Auswertung visuell evozierter EEG-Potentiale bei Verdacht auf multiple Sklerose. J Neurol 213, 97-103

Lehmann D, Skrandies W (1979) Multichannel evoked potential fields show different properties of human upper and lower hemi-retinal systems. Exp Brain Res 35, 151-159

Lehmann D, Skrandies W (1980) Reference-free identification of components of checkerboard-evoked multichannel potential fields. Electroencephalogr Clin Neurophysiol 48, $609-621$

Lehmann D, Skrandies W (1984) Spatial analysis of evoked potentials in man: a review. Progr Neurobiol 23, 227-250

Lehmann D, Meles HP, Mir Z (1977) Average multichannel EEG potential fields evoked from upper und lower hemiretina: latency differences. Electroencephalogr Clin Neurphysiol $43,725-731$

Lehmann D, Darcey TM, Skrandies W (1982) Intracerebral and scalp fields evoked by hemiretinal checkerboard reversal, and modeling of their dipole generators. In: Courjon $\mathrm{J}$, Maugiere $\mathrm{F}$, Revol $\mathrm{M}$ (eds) Clinical applications of evoked potentials in neurology. Raven, New York, pp 41-48

Lesèvre N (1972) Potentiels évoqués par des patterns chez l'homme: influence des variables caracterisant le stimulus et sa position dans le champ visuel. In: Fessard A, Lelord G (eds) Activités evoquées et leur conditionnement. INSERM, Paris, pp 1-22

Lesèvre N, Joseph JP (1979) Modifications of the pattern-evoked potential (PEP) in relation to the stimulated part of the visual field. Electroencephalogr Clin Neurophysiol 47, $183-203$

Lichtenstein M, White CT (1961) Relative visual latency as a function of retinal locus. J Opt Soc Am 51, 1033-1034

Lindsley DB, Lansing RW (1956) Flicker and two-flash fusional threshold and EEG, Am Psychol 11, 433

Linsenmeyer RA, Steinberg RH (1982) Origin and sensitivity of the light peak in the intact cat eye. J Physiol 331, 653-673 
Lipkin BS (1962) Monocular flicker discrimination as a function of the luminance and area of contralateral steady light: I. Luminance. II. Area. J Opt Soc Am 52:1287-1295, $1296-1300$

Low FN (1943) The peripheral visual acuity of 100 subjects. Am J Physiol 140, 83-88

Lundh BL, Lennerstrand G, Derefeldt G (1983) Central and peripheral normal contrast sensitivity for static and dynamic sinusoidal gratings. Acta Ophthalmol 61, 171-182

Luria SM, Kinney JA (1970) Underwater vision. Science 167, 1454-1461

MacKay DM (1984a) Source density analysis of scalp potentials during evaluated action. I. Coronal distribution. Exp Brain Res 54, 73-85

MacKay DM (1984b) Source density analysis of scalp potentials during evaluated action. II. Lateral coronal distributions. Exp Brain Res 54, 86-94

Maffei L (1982) Electroretinographic and visual cortical potentials in response to alternating gratings. Ann NY Acad Sci 388, 1-10

Maffei L, Fiorentini A (1981) Electroretinographic responses to alternating gratings before and after section of the optic nerve. Science 211, 953-955

Maffei L, Fiorentini A, Bisti S, Holländer H (1985) Pattern ERG in the monkey after section of the optic nerve. Exp Brain Res 59, 423-425

Magrotti E, Cosi V, Borutti G (1980) Differenze funzionali tra emicampi visivi superiore ed inferiore per stimoli non strutturanti. Boll Soc Ital Biol Sper 56, 416-422

Martin GR, Muntz WRA (1979) Retinal oil droplets and vision in the pigeon (Columba liva). In: Granda AM, Maxwell JF (eds) Neural mechanisms of behavior in the pigeon. Plenum, New York, pp 307-325

Matelli M, Olivieri MF, Saccani A, Rizzoloatti G (1983) Upper visual space neglect and motor deficits after section of the midbrain commissures in the cat. Behav Brain Res $10,263-285$

McAlpine D, Lumsden CE, Acheson ED (1972) Multiple sclerosis: a reappraisal. Williams and Wilkins, Baltimore

Millodot M, Lamont A (1974) Peripheral visual acuity in the vertical plane. Vision Res 14, $1497-1498$

Mitzdorf U (1985) Current source-density method and application in cat cerebral cortex: investigation of evoked potentials and EEG phenomena. Physiol Rev 65, 37-100

Mitzdorf U, Singer W (1978) Prominent excitatory pathways in the cat visual cortex (A 17 and $A$ 18): a current source density analysis of electrically evoked potentials. Exp Brain Res 33, 371-394

Murray I, MacCana F, Kulikowski JJ (1983) Contribution of two movement detecting mechanisms to central and peripheral vision. Vision Res 23, 151-159

Nachmias J (1959) Two-dimensional motion of the retinal image during monocular fixation. J Opt Soc Am 49, $901-908$

Nagata T, Hayashi Y (1984) The visual field representation of the rat ventral lateral geniculate nucleus. J Comp Neurol 227, 582-588

Newman RP, Kinkel WR, Jacobs L (1984) Altitudinal hemianopia caused by occipital infarctions. Arch Neurol 41, 413-418

Niemeyer G (1975) The function of the retina in the perfused eye. Doc Ophthalmol 35 , $53-116$

Nuboer JFW, Wortel JF (1985) Wavelength discrimination in the lower and upper visual field of the pigeon. J Physiol 366, 95P

Nunez P (1981) Electric fields of the brain. Oxford University Press, New York

$\emptyset$ sterberg G (193.5) Topography of the layer of rods and cones in the human retina. Acta Ophthalmol [13 Suppl. 6]: 1-102

Oyster CW, Takahashi ES, Cilluffo M, Brecha NC (1985) Morphology and distribution of tyrosine hydroxylase-like immunoreactive neurons in the cat retina. Proc Natl Acad Sci $82,6335-6339$

Payne WH (1965) Visual reaction times on a circle about the fovea. Science 155, 481-482 
Pease VP, Sticht TG (1965) Reaction time as a function of onset and offset stimulation of the fovea and periphery. Percept Mot Skills 20, 549-554

Phelps ME, Kuhl DE, Mazziotta JC (1981) Metabolic mapping of the brain's response to visual stimulation: studies in humans. Science $211,1445-1448$

Phillips G (1933) Perception of flicker in lesions of the visual pathways. Brain 56, 464-478

Poffenberger AT (1912) Reaction time to retinal stimulation with special reference to the time lost in conduction through nerve centers. Arch Psychol 3, 1-73

Poggio GF, Fischer B (1977) Binocular interaction and depth sensitivity in striate and prestriate cortex of behaving rhesus monkey. J Neurophysiol 40, 1392-1405

Presson J, Moran J, Gordon B (1983) Effects of eye rotation on visually guided behavior. J Neurophysiol 50, 631-643

Prinz W (1984) Attention and sensitivity in visual search. Psychol Res 45, 355-366

Quingley HA, Flower RW, Addicks EM, McLeod DS (1980) The mechanism of optic nerve damage in experimental acute intraocular pressure elevation. Invest Ophthalmol 19, $505-517$

Rains JD (1963) Signal luminance and position effects in human reaction time. Vision Res 3, 239-251

Regan D, Silver R, Murray TJ (1977) Visual acuity and contrast sensitivity in multiple sclerosis - hidden visual loss. Brain 100, 563- 579

Rémond A, Lesèvre N, Joseph JP, Rieger H, Lairy GC (1969) The alpha average: I. Methodology and description. Electroencephalogr Clin Neurophysiol 26, 245-265

Reuter TE, White RH, Wald G (1971) Rhodopsin and porphyropsin in the adult bullfrog retina. J Gen Physiol 58, 351-371

Riemslag FCC, Ringo JL, Spekreijse H, Verduyn Lunel HF (1985) The luminance origin of the pattern electroretinogram in man. J Physiol 363, 191-209

Riggs LA, Johnson EP, Schick AML (1964) Electrical responses of the human eye to moving stimulus patterns. Science 144,567

Scalia F (1976) The optic pathway of the frog: nuclear organization and connections. In: Llinas R, Precht W (eds) Frog neurobiology. Springer Berlin Heidelberg New York, pp $386-406$

Schade OH (1956) Optical and photoelectric analog of the eye. J Opt Soc Am 46, 721 - 739

Schmidt M, Wässle H, Humphrey M (1985) Number and distribution of putative cholinergic neurons in the cat retina. Neurosci Lett 59, 235-240

Schneider MR (1972) A multistage process for computing dipolar sources of EEG discharges from surface information. IEEE Trans Biomed Eng 19, 1-12

Schneider MR (1974) Effect of inhomogeneities on surface signals coming from a cerebral dipole source. IEEE Trans Biomed Eng 21, 52-54

Schwartz EL, Christman DR, Wolf AP (1984) Human primary visual cortex topography imaged via positron tomography. Brain Res 294, 225-230

Seiple WH, Siegel IM (1983) Recording the pattern electroretinogram: a cautionary note. Invest Ophthalmol 24, 796-798

Seneviratne KN (1963) The representation of the visual field on the subcortical centers of the cat and rabbit. PhD thesis, Edinburgh

Seneviratne KN, Whitteridge D (1962) Visual evoked responses in the lateral geniculate nucleus. Electrocephalogr Clin Neurophysiol 14, 785

Shickman GM (1981) Time-dependent functions in vision. In: Moses RA (ed) Adler's physiology of the eye. Mosby, St. Louis, pp 663-713

Shurong W, Kun Y, Yinting W (1981) Visual field topography and binocular responses in frog's nucleus isthmi. Scientia Sin [B] 24, 1292-1301

Sidman RD, Giambalvo V, Allison T, Bergey P (1978) A method for localization of sources of human cerebral potentials evoked by sensory stimuli. Sensory Proc 2, 116-129

Simonson E (1958) Contralateral glare effect on the fusion frequency of flicker. Arch Ophthalmol 60, 995-999 
Skrandies W (1981) Latent components of potentials evoked by visual stimuli in different retinal locations. Int $\mathrm{J}$ Neurosci $14,77-84$

Skrandies W (1983) Information processing and evoked potentials: topography of early and late components. Adv Biol Psychiatr 13,1-12

Skrandies W (1984a) Differences of visual evoked potential latencies and topographies depending on retinal location and presentation mode. Pflügers Arch 400, R31

Skrandies W (1984b) Scalp potential fields evoked by grating stimuli: effects of spatial frequency and orientation. Electroencephalogr Clin Neurophysiol 58, 325-332

Skrandies W (1985a) Critical flicker fusion and double flash discrimination in different parts of the visual field. Int $\mathrm{J}$ Neurosci $25,225-231$

Skrandies W (1985b) Human contrast sensitivity: regional retinal differences. Hum Neurobiol 4, 95-97

Skrandies W (1986a) Visual evoked potential topography: methods and results. In: Duffy FH (ed) Topographic mapping of brain electrical activity. Butterworth, Boston, pp 7-28

Skrandies W (1986b) Temporal summation of stereoscopic visual stimuli: brain electric components and subjective perception. In: Rohrbaugh JW, Johnson R, Parasuraman R (eds) Research reports of EPIC VIII conference, Stanford, pp 397-399

Skrandies W, Baier M (1986) Activity of human pigment epithelium shows differences between upper and lower retinal areas. Vision Res 26, 577-581

Skrandies W, Gottlob I (1986) Alterations of visual contrast sensitivity in Parkinson's Disease. Hum Neurobiol 5, 255-259

Skrandies W, Lehmann D (1982a) Occurrence time and scalp location of components of evoked EEG potential fields. In: Herrmann WM (ed) Electroencephalography in drug research. Fischer, Stuttgart, pp 183-192

Skrandies W, Lehmann D (1982b) Spatial principal components of multichannel maps evoked by lateral visual half-field stimuli. Electroencephalogr Clin Neurophysiol 54, $662-667$

Skrandies W, Vomberg HE (1985) Stereoscopic stimuli activate different cortical neurones in man: electrophysiological evidence. Int J Psychophysiol 2, 293-296

Skrandies W, Richter M, Lehmann D (1980) Checkerboard evoked potentials: topography and latency for onset, offset, and reversal. Progr Brain Res 54, 291-295

Skrandies W, Wässle H, Peichl L (1978) Are field potentials an appropriate method for demonstrating connections in the brain? Exp Neurol 60, 509-521

Skrandies W, Chapman RM, McCrary JW, Chapman JA (1984) Distribution of latent components related to information processing. Ann NY Acad Sci 425, 271-277

Soso MJ, Lettich E, Belgum JH (1980) Pattern-sensitive epilepsy. II: Effects of pattern orientation and hemifield stimulation. Epilepsia 21, 313-323

Southall JPC (1962) Helmholtz's treatise on physiological optics. Dover, New York

Spalding JMK (1952) Wounds of the visual pathway. J Neurol Neurosurg Psychiatr 15, $169-183$

Sperry RW (1964) The great cerebral commissure. Sci Am 210, 42-52

Sperry RW (1968) Hemisphere deconnection and unity in conscious awareness. Am Psychol 23, 723-733

Standage GP, Benevento LA, The organization of connections between the pulvinar and visual area MT in the macaque monkey. Brain Res 262, 288-294

Starr A, Achor J (1975) Auditory brainstem responses in neurological disease. Arch Neurol $32,761-768$

Stone J, Johnston E (1981) The topography of primate retina: a study of the human, bushbaby, and New- and Old-World monkeys. J Comp Neurol 196, 205-223

Sutton S, Braren M, Zubin J, John ER (1965) Evoked potential correlates of stimulus uncertainty. Science $150,1187-1188$

Talairach J, Szikla G (1967) Atlas of stereotaxic anatomy of the telencephalon. Masson, Paris 
Täumer R (ed) (1976) Electro-oculography - its clinical importance. Karger, Basel. Bibliotheca Ophthalmologica, Vol 85

ten Doesschate J (1946) Visual acuity and distribution of percipient elements on the retina. Ophthalmologica $112,1-18$

Teuber H-L, Battersby WS, Bender MB (1960) Visual field defects after penetrating missile wounds of the brain. Harvard University Press, Cambridge

Teuber ML (1974) Sources of ambiguity in the prints of Maurits C. Escher. Scie. Am. 231 (No 1), 90-104

Torrealba F, Guillery RW, Eysel U, Polley EH, Mason CA (1982) Studies of retinal representations within the cat's optic tract. J Comp Neurol 211, 377-396

Tusa RJ, Palmer LA, Rosenquist AC (1978) The retinotopic organization of area 17 (striate cortex) in the cat. J Comp Neurol 177, 213-236

Valeton JM, van Norren D (1982) Intraretinal recording of slow electrical responses to steady illumination in monkey: isolation of receptor responses and the origin of the light peak. Vision Res 22, 393-399

van Buren A (1963) The retinal ganglion cell layer. Thomas, Springfield

van de Grind WA, Grüsser O-J, Lunkenheimer H-U (1973) Temporal transfer properties of the afferent visual system. Psychophysical, neurophysiological and theoretical investigations. In: Jung $R$ (ed) Handbook of sensory physiology, Vol VII/3A. Springer, Berlin Heidelberg New York, pp 431-573

van der Waerden BL (1971) Mathematische Statistik, 3rd ed. Springer, Berlin Heidelberg New York

van Essen DC (1985) Functional organization of primate visual cortex. In: Peters A, Jones EG (eds) Cerebral cortex, Vol 3. Plenum, New York, pp 259-329

van Essen DC, Maunsell JHR, Bixby JL (1981) The middle temporal visual area in the macaque: myeloarchitecture, connections, functional properties and topographic organization. J Comp Neurol 199, 293-326

van Essen DC, Newsome WT, Maunsell JHR (1984) The visual field representation in striate cortex of the macaque monkey: asymmetries, anisotropies, and individual variability. Vision Res $24,429-448$

van Essen DC, Newsome WT, Maunsell JHR, Bixby JL (1986) The projections from striate cortex (V1) to areas V2 and V3 in the macaque monkey: asymmetries, areal boundaries, and patchy connections. J Comp Neurol 244, 451-480

Vaney DI (1985) The morphology and topographic distribution of AII amacrine cells in the cat retina. Proc R Soc Lond [Biol] 224, 475-488

Vaney DI, Hughes A (1976) Rabbit optic nerve: fibre diameter spectrum, fibre count, and comparison with a retinal ganglion cell count. J Comp Neurol 170, 241-251

Vomberg HE, Skrandies W (1985) Untersuchung des Stereosehens im ZufallspunktmusterVECP: Normbefunde und klinische Anwendung. Klin Monatsbl Augenheilkd 187, $205-208$

von der Heydt R, Hänni P, Dürsteler M, Peterhans E (1981) Neuronal responses to stereoscopic stimuli in the alert monkey - a comparison between striate and prestriate cortex. Pflügers Arch 391, R34

von Helmholtz H (1853) Über einige Gesetze der Vertheilung elektrischer Ströme in körperlichen Leitern, mit Anwendung auf die thierelektrischen Versuche. Ann Phys Chemie 29: $211-233,353-377$

von Helmholtz $\mathrm{H}$ (1910) Handbuch der physiologischen Optik, Vol 3, 3rd edn. Voss, Hamburg

Wässle H, Peichl L, Boycott BB (1978) Topography of horizontal cells in the retina of the domestic cat. Proc R Soc Lond [Biol] 203, 269-291

Wernicke C (1874) Der aphasische Symtomenkomplex. Cohne und Weigert, Breslau

Wertheim T (1894) Ưber die indirekte Sehsschärfe. Z Psychol Physiol Sinnesorg 7, $172-187$ 
Whitteridge D (1973) Projection of optic pathways to the visual corex. In: Jung R (ed) Handbook of sensory physiology, Vol VII/3B. Springer, Berlin Heidelberg New York, pp 247-268

Wilson FN, Bayley RH (1950) The electric field of an eccentric dipole in a homogenous spherical conducting medium. Circulation 1, 84-92

Woodworth RS (1938) Experimental psychology. Holt, New York

Woodworth RS, Schlossberg H (1955) Experimental psychology, 3rd edn. Holt, New York

Yanashima K (1982) Surface distribution of steady-state cortical potentials evoked by visual half-field stimulation. Gräfes Arch Ophthalmol 218, 118-123

Yarbus AL (1967) Eye movements and vision. Plenum, New York

Zihl J, von Cramon D (1986) Zerebrale Sehstörungen. Kohlhammer, Stuttgart

Zubek JP, Bross M (1972) Depression and later enhancement of the critical flicker frequency during prolonged monocular deprivation. Science 176, 1045-1047

Zubek JP, Bross M (1973) Changes in critical flicker frequency during and after fourteen days of monocular deprivation. Nature 241, 288-290 